\title{
Rhizobium sp. Strain NGR234 and $R$. fredii USDA257 Share Exceptionally Broad, Nested Host Ranges
}

\author{
Steven G. Pueppke ${ }^{1,2}$ and William J. Broughton ${ }^{2}$ \\ ${ }^{1}$ Department of Plant Pathology, University of Missouri, Columbia 65211, U.S.A., and ${ }^{2}$ Laboratoire de Biologie \\ Moléculaire des Plantes Supérieures, Université de Genève, 1 ch. de l'Impératrice, 1292 Chambésy/Genève, \\ Switzerland \\ Accepted 2 November 1998.
}

Genetically, Rhizobium sp. strain NGR234 and $R$. fredii USDA257 are closely related. Small differences in their nodulation genes result in NGR234 secreting larger amounts of more diverse lipo-oligosaccharidic Nod factors than USDA257. What effects these differences have on nodulation were analyzed by inoculating 452 species of legumes, representing all three subfamilies of the Leguminosae, as well as the nonlegume Parasponia andersonii, with both strains. The two bacteria nodulated $P$. andersonii, induced ineffective outgrowths on Delonix regia, and nodulated Chamaecrista fasciculata, a member of the only nodulating genus of the Caesalpinieae tested. Both strains nodulated a range of mimosoid legumes, especially the Australian species of Acacia, and the tribe Ingeae. Highest compatibilities were found with the papilionoid tribes Phaseoleae and Desmodieae. On Vigna spp. (Phaseoleae), both bacteria formed more effective symbioses than rhizobia of the "cowpea" ( $V$. unguiculata) miscellany. USDA257 nodulated an exact subset (79 genera) of the NGR234 hosts (112 genera). If only one of the bacteria formed effective, nitrogen-fixing nodules it was usually NGR234. The only exceptions were with Apios americana, Glycine max, and G. soja. Few correlations can be drawn between Nod-factor substituents and the ability to nodulate specific legumes. Relationships between the ability to nodulate and the origin of the host were not apparent. As both $P$. andersonii and NGR234 originate from Indonesia/Malaysia/Papua New Guinea, and NGR234's preferred hosts (Desmodiinae/ Phaseoleae) are largely Asian, we suggest that broad host range originated in Southeast Asia and spread outward.

Fuchsius (1542) was probably the first to publish drawings of legume root nodules. Unfortunately, nodules were not mentioned in the accompanying text, and subsequent publications assigned them to everything from disease responses to

Corresponding author: W. J. Broughton, LBMPS, Université de Genève, $1 \mathrm{ch}$. de l'Impératrice, 1292 Chambésy/Genève, Switzerland. Telephone: (0041) (22) 90617 40; Fax: (0041) (22) 90617 41; E-mail: <broughtw@sc2a.unige.ch>

Present address of Steven G. Pueppke: College of Agricultural, Consumer, and Environmental Sciences, University of Illinois, 1301 West Gregory Drive, Urbana 61801, U.S.A. storage organs (see Fred et al. 1932). Frank (1879) showed that sterilizing soil prevented nodule formation, while Hellriegel (1886) and Hellriegel and Wilfarth (1888) demonstrated that nodule formation results from an infection. Proof that bacteria were the causative agents came from Beyerinck (1888a, $1888 \mathrm{~b}, 1888 \mathrm{c}, 1888 \mathrm{~d}, 1888 \mathrm{e}$ ), who prepared pure cultures of the nodule occupants and used them to infect legumes (Beyerinck 1890). Finally, Prazmowski (1889, 1890) inoculated Pisum sativum with pure cultures and showed that the bacteria penetrate legumes via infection threads in root hairs.

Specificity in legume-Rhizobium associations was also apparent at the end of the last century. Nobbe et al. (1891, 1895) found that bacteria isolated from $P$. sativum nodules were unable to nodulate plants belonging to the tribes Genisteae and Hedysareae. Ever since Hiltner and Störmer (1903) tried to classify rhizobia from various plant sources, numerous taxonomic proposals have been made (e.g., Fred et al. 1932). All strongly emphasized the host from which the rhizobia were isolated, which led to numerous problems (Wilson 1939; Lim and Burton 1982; Trinick 1982). Foremost among these is the unwieldy size of groups such as the "cowpea" miscellany, which originally had as its nucleus 21 legume genera (41 species). By definition, each of these "cowpea" rhizobia can nodulate Vigna unguiculata in addition to the host from which it was isolated. An additional 117 species were added to the group in the mid 1930s (Carroll 1934; Allen and Allen 1936). Two decades later, the majority of all nodulated legumes were included in this untidy and largely unworkable assemblage (Norris 1956).

Interestingly, work on the molecular basis of host specificity also began about 100 years ago. Hiltner (1900) showed that aqueous, bacteria-free filtrates from mature $P$. sativum nodules contained a substance that induced root-hair formation and deformation of the root hairs (Had) of P. sativum. Although many others sought these "Had-factors," the research only culminated in the report of Lerouge et al. (1990), who showed that the substances responsible for deformation are $\mathrm{N}$-acylated oligomers of $\mathrm{N}$-acetyl-D-glucosamine (see Relić et al. 1993). Since then, the "Had-factor" structures (now called Nod factors as they are the products of the nodulation genes; see Roche et al. 1991b) of a number of Azorhizobium/ Bradyrhizobium/ Mesorhizobium/ Sinorhizobium/ Rhizobium species have been elucidated (see Schultze et al. 1994; Fellay et al. 1995b; Dénarié et al. 1996; Long 1996; Spaink 1996; Hanin et al. 1999; Cohn et al. 1998). 
As Nod factors are responsible for root-hair curling (Hac), the induction of nodulation (Hai), and the entry of rhizobia into the roots, it should be possible to define host-range differences between rhizobia in terms of the genes involved in Nod-factor production.

Our work is concerned with the control of broad host range in legume-Rhizobium associations. As model rhizobia, we use Rhizobium sp. strain NGR234 (W. J. B.), and $R$. fredii USDA257 (S. G. P.). NGR234 was the only fast-growing strain among 30 isolates prepared from Lablab purpureus nodules in Papua New Guinea (soil pH 8.5) by M. J. Trinick (in 1965). Shortly afterward, Trinick recognized the potential of NGR234 and generously distributed it a decade in advance of publication (see Trinick 1980). Thus, Broughton and Dilworth (1971) inoculated V. unguiculata with either NGR234 or a Bradyrhizobium sp. to confirm that the plant encodes the globin portion of the leghemoglobin molecule. Reports followed on both the bacterium (Broughton et al. 1972) and development of $V$. unguiculata nodules induced by NGR234 (Broughton et al. 1978). Interest in NGR234 gradually increased following Trinick's comparison with other fastgrowing rhizobia (Trinick 1980), and the nodulation characteristics of various derivatives as well as spontaneous resistance mutants including ANU237 (wild type), ANU240 (Sm $\left.{ }^{\mathrm{r}}\right)$, ANU265 (cured of the symbiotic plasmid), ANU280 $\left(\mathrm{Sm}^{\mathrm{r}}\right.$ Rif ${ }^{\mathrm{r}}$ ), and MPIK3030 $\left(\mathrm{Sm}^{\mathrm{r}}\right)$ have been published (Kondorosi et al. 1982; Morrison et al. 1983; Pankhurst et al. 1983a, 1983b; Wong et al. 1983; Morrison et al. 1984; Broughton et al. 1984; Bachem et al. 1985; Broughton et al. 1985; Bachem et al. 1986; Bassam et al. 1986; Broughton et al. 1986; Dilworth et al. 1986; Horvath et al. 1987; Lewin et al. 1987a, 1987b; Nayudu and Rolfe 1987; Stanley et al. 1988; Williams et al. 1988; Wong et al. 1988). In the 10 years since the last of these reports, there has been an explosion of interest in NGR234 and related strains (see Perret, Jabbouri, and Broughton, in press, and Perret et al., in press). Although NGR234 has a short generation time and a G+C content characteristic of Rhizobium spp. (Broughton et al. 1972), its single subpolar flagellum (Padmanabhan et al. 1989) is more representative of those found on Bradyrhizobium spp.

In contrast, $R$. fredii USDA257 was isolated from a wild soybean (Glycine soja) plant growing near Wuking, China (Keyser and Griffin 1987; Keyser et al. 1982). Although generally considered to be a soybean symbiont (e.g., Dénarié et al. 1992), $R$. fredii nodulates a number of additional legume species (Broughton et al. 1984; Heron and Pueppke 1984; Keyser et al. 1982; Morrison et al. 1986; Stowers and Eaglesham 1984).

Data from DNA subtraction hybridizations confirmed that NGR234 and USDA257 are phylogenetically closely related, and share most of their genomic background (Perret et al. 1994). Homologies with insertion sequences (IS) and the absence from the USDA257 genome of the NGRRS-1 transposon-like repeat (Perret et al. 1997) suggest that many of the sequences "unique" to NGR234 are mobile elements that have accumulated since both bacteria diverged. Comparisons of the host ranges of NGR234 and USDA257 should thus allow correlations to be made between those genes (and the Nod-factor substituents) that are unique to NGR234 and its ability to nodulate specific plants. Furthermore, as the geographic and temporal origins of the two rhizobia are substantially differ- ent, nodulation capacity can be evaluated in terms of legume distribution and taxonomy. For these reasons, we tested the ability of both strains to nodulate a wide spectrum of plants.

\section{RESULTS}

\section{Reproducibility of nodulation tests.}

Rigorously controlled nodulation tests would have involved optimizing (i) growth conditions for each individual legume, (ii) inoculation conditions, especially by including a "benchmark" strain (i.e., one chosen for the particular plant because of its high nitrogen-fixing capacity), and (iii) harvest times for each legume. Given the number of legumes tested and the paucity of information available on many of them (including their rhizobial requirements), this was not practical. Rather, standardized conditions were developed that, though probably suboptimal for some plants, permitted reproducible tests in disparate laboratories.

Glycine max presents a good example of the kinds of problems presented by suboptimal growth conditions. Trinick (1980) reported that NGR234 effectively nodulates (i.e., is Fix $^{+}$) on $G$. max but did not specify the cultivar. Broughton et al. (1984) found that NGR234 formed ineffective nodules on G. $\max \mathrm{cv}$. Caloria $\left(\mathrm{Nod}^{+}\right)$, but that it failed to nodulate $\mathrm{cv}$. Peking. Both sets of experiments were performed in large, "Leonard jar" assemblies (Leonard 1943) housed in glasshouses. Balatti and Pueppke (1990) concluded that G. max cv. McCall is not infected by NGR234 in plastic growth pouches. In a later study, Balatti et al. (1995) stated that "under uniform growth conditions in vermiculite, Rhizobium sp. NGR234 did not form normal Fix ${ }^{+}$nodules on any of a wide variety of soybean cultivars." This set of inoculation tests was performed on 89 different $G$. max cultivars grown in the same type of Magenta jar assemblies described here. It thus seems unavoidable that both abiotic and biotic factors (particularly cultivar and inoculum dosage) influence the spectrum of plants nodulated. In this sense, nodulation is a conditional phenotype.

\section{Nodulation status of the Leguminosae nodule type.}

About $57 \%$ of the legume genera have been examined for nodulation (approximately 3,500 species, 20\% of the Leguminosae). Of these, only $23 \%$ of Caesalpinioid species have been found to nodulate, versus $90 \%$ in the Mimosoideae and $97 \%$ in the Papilionoideae (de Faria et al. 1989). It was thus important to compare the nodulation ability of the two rhizobia with the capacity of each individual legume to nodulate. To do this, a collation of the nodulation status of all the species tested (taken from Lim and Ng 1977; Allen and Allen 1981; de Faria et al. 1989; de Souza Moreira et al. 1992; Whitty et al. 1994; Athar 1993, 1996a, 1996b, 1997; and J. I. Sprent, personal communication) is presented in Table 1. Taxonomic entities not known to nodulate are shaded in the table.

Since nodule structure and function are largely determined by the plant (Dart 1977; Corby et al. 1983; Sprent and Sprent 1990), the nodulation capacities of the two bacteria were also compared with the types of nodules elicited. For simplicity, only three different types of nodule were recognized: Aeschynomenoid, determinate, and indeterminate (marked in pink, blue, and green, respectively, in Table 1). This information was taken from the publications of Corby et al. (1983), Crisp and Weston (1987), Sprent et al. (1989), Corby (1988), 
James et al. (1993), Sutherland et al. (1994), Cordeiro and Sprent (1996), and Harrier et al. (1997), and from J. I. Sprent (personal communication).

Nodules formed on Lupinus spp. (Genisteae), which do not fit easily into any of these classes, were not classified. Aeschynomenoid nodules, which are a feature of the Aeschynomeneae (but also found in the Dalbergieae), are characterized by a direct, intercellular invasion process (Allen and Allen 1940; Dart 1977; Chandler 1978; Chandler et al. 1982). Rather than entering through the root hairs of, e.g., Arachis spp. and Stylosanthes spp., rhizobia penetrate the intercellular spaces. After the bacteria have gained entry into the root, they penetrate the cortical cells in a process that resembles pinocytosis (Meijer and Broughton 1982). A unique feature of Aeschynomenoid nodules is that the bacteroidcontaining cortical cells continue to divide. Although a certain amount of dimorphism exists (Corby et al. 1983), determinate nodules (see cover) generally lack an apical meristem and are short-lived; the vascular strands fuse at the apex and they usually export ureides to the xylem (Sprent 1980). On the other hand, indeterminate nodules possess an apical meristem and export amino acids and amides (Corby et al. 1983). Although both types of nodules are found in the Papilionoideae, the indeterminate "caesalpinioid" type is the only one found in the Caesalpinioideae and Mimosoideae, suggesting that it is the plesiomorphic type (Doyle et al. 1997).

\section{Legumes nodulated by NGR234 and USDA257.}

In all, the ability of Rhizobium sp. strain NGR234 and $R$. fredii USDA257 to form root nodules was tested on a total of 452 legume species (Table 2), distributed among 194 genera in all three subfamilies of the Leguminosae (Polhill and Raven 1981; Corby et al. 1983; Polhill 1994). Twenty-four of the 153 Caesalpinioid genera, including all four tribes in this subfamily, were tested. The corresponding figures for the subfamily Mimosoideae are 20 out of 65 genera, distributed within four of the five recognized tribes. Far more nodulation tests were performed with papilionaceous genera, however. One hundred and fifty of 448 genera were examined, including every tribe except the Carmichaelieae, Dipterygeae, Euchresteae, and Liparieae. Among the Papilionoideae seven of the eight subtribes of the Phaseoleae, the largest tribe of the Leguminosae, and one that contains many species of commercial importance, were tested.

\section{Subfamily Caesalpinioideae.}

Chamaecrista, a mostly tropical and subtropical tribe, is the only large exception to the rule that Caesalpinioid legumes are not known to nodulate (Sprent 1994) (Table 1). It was therefore perhaps not surprising that USDA257 was $\mathrm{Nod}^{+}$and NGR234 Fix ${ }^{+}$on C. fasciculata (Table 1). Although Delonix regia is generally considered to be refractive to rhizobia (Allen and Allen 1981), Lim and Ng (1977) reported the presence of small, Fix ${ }^{-}$nodules on this plant growing in Singapore. As NGR234 and USDA257 elicited similar nodules, we were able to confirm their observations.

\section{Subfamily Mimosoideae.}

NGR234 nodulated 28 of 53 species in this subfamily and in 16 cases the nodules were Fix ${ }^{+}$. Nodulation by USDA257 was restricted to 18 of these species, but nitrogen was fixed in combination with only seven (Table 1). Thus, with the exception of the genera Calliandra and Leucaena, the nodulating abilities of the two strains for mimosoid legumes are quite similar. Both strains nodulated hosts such as Albizia spp., Enterolobium contortisiliquum, and Desmanthus illinoensis, all of which are thought to have symbiotic preferences for slowgrowing bradyrhizobia (Allen and Allen 1981). The production of fully $\mathrm{Fix}^{+}$nodules by USDA257 on Albizia lebbeck and $A$. procera is noteworthy, because it shows that $R$. fredii can also effectively nodulate leguminous trees.

Interestingly, almost all the species of the Acacia subgenus Heterophyllum are Australian, and all tested here (A. auriculiformis, A. cyanophylla, A. mangium, A. mearnsii, A. pendula, A. retionodes, and A. saligna) nodulated with NGR234. Furthermore, with the exception of $A$. pendula, all also formed nodules with USDA257. Of the subgenus Aculeiferum (A. aroma, A. ataxacantha, A. bonariensis, and A. macracantha), only the two Argentinian species (A. bonariensis and A. macracantha) nodulated, and then only ineffectively, with NGR234. None of the African species nodulated with either strain. A. ataxacantha is believed not to nodulate, in common with a number of close relatives within the section Monocanthea of the subgenus Aculeiferum (Harrier et al. 1997).

Acacia spp. are known to form symbioses with both rhizobia and bradyrhizobia (Dreyfus and Dommergues 1981). Recently, the rhizobial requirements of a number of them have been shown to be even more diverse and include Rhizobium saheli and $R$. teranga bv. sesbaniae, species that produce arabinosylated and fucosylated Nod factors similar to those produced by Azorhizobium caulinodans (Lorquin et al. 1997a, 1997b). Although inoculation with NGR234 gave Fix ${ }^{+}$nodules with four Acacia spp. (A. auriculiformis, A. pendula, A. retinodes, and $A$. saligna), none of the 15 species tested fixed nitrogen with USDA257. Perhaps this indicates that NGR234 possesses host-specific nitrogen-fixation genes for Acacia that

Table 2. Analyses of the ability of Rhizobium sp. strain NGR234 and R. fredii USDA257 to nodulate members of the plant family Leguminosae ${ }^{\text {a }}$

\begin{tabular}{|c|c|c|c|c|c|c|c|c|}
\hline \multirow[b]{2}{*}{ Subfamily } & \multicolumn{2}{|c|}{ Tribes } & \multicolumn{2}{|c|}{ Genera } & \multicolumn{2}{|c|}{ Species } & \multicolumn{2}{|c|}{ Total species nodulated } \\
\hline & USDA & NGR & USDA & NGR & USDA & NGR & USDA & NGR \\
\hline Caesalpinoideae & $2^{\mathrm{N}}$ & $1^{\mathrm{N}}, 1^{\mathrm{F}}$ & $2^{\mathrm{N}}$ & $1^{\mathrm{N}}, 1^{\mathrm{F}}$ & $2^{\mathrm{N}}$ & $1^{\mathrm{N}}, 1^{\mathrm{F}}$ & & \\
\hline Number tested & & 4 & & 24 & & 46 & & \\
\hline Mimosoideae & $1^{\mathrm{N}}, 2^{\mathrm{F}}$ & $3^{\mathrm{F}}$ & $4^{\mathrm{N}}, 6^{\mathrm{F}}$ & $2^{\mathrm{N}}, 10^{\mathrm{F}}$ & $11^{\mathrm{N}}, 7^{\mathrm{F}}$ & $12^{\mathrm{N}}, 16^{\mathrm{F}}$ & & \\
\hline Number tested & & 4 & & 20 & & 53 & & \\
\hline Papilionoideae & $9^{\mathrm{N}}, 9^{\mathrm{F}}$ & $3^{\mathrm{N}}, 18^{\mathrm{F}}$ & $34^{\mathrm{N}}, 33^{\mathrm{F}}$ & $37^{\mathrm{N}}, 61^{\mathrm{F}}$ & $56^{\mathrm{N}}, 59^{\mathrm{F}}$ & $83^{\mathrm{N}}, 119^{\mathrm{F}}$ & & \\
\hline Number tested & & 26 & & 150 & & 353 & & 452 \\
\hline Totals by taxonomic group & $12^{\mathrm{N}}, 11^{\mathrm{F}}$ & $4^{\mathrm{N}}, 22^{\mathrm{F}}$ & $40^{\mathrm{N}}, 39^{\mathrm{F}}$ & $40^{\mathrm{N}}, 72^{\mathrm{F}}$ & $69^{\mathrm{N}}, 66^{\mathrm{F}}$ & $96^{\mathrm{N}}, 136^{\mathrm{F}}$ & $135(30 \%)$ & $232(51 \%)$ \\
\hline
\end{tabular}

${ }^{a}$ A total of 452 species distrbuted among the three subfamilies of the Leguminosae were tested. Superscripts represent the number of taxa that formed $\mathrm{Nod}^{+}$or Fix ${ }^{+}$nodules. Both bacteria were also $\mathrm{Nod}^{+}$on Parasponia andersonii (Ulmaceae). 
are either absent or inactive in USDA257, an observation that is consistent with the fact that Acacia prefers sulfated Nod factors (Lortet et al. 1996).

\section{Subfamily Papilionoideae.}

Both NGR234 and USDA257 are widely compatible with species of this subfamily (Table 1). USDA257 nodulated 115 Papilionoid species (out of 359 tested), and in $52 \%$ of the cases, nitrogen was fixed. NGR234 nodulated the same 115 species, as well as an additional 87 species distributed among
24 tribes of this subfamily $(=57 \%$ of all Papilionoideae tested). One hundred and nineteen of the 202 species (59\%) nodulated by NGR234 fixed atmospheric nitrogen.

Although our data on some tribes are limited, analysis of Table 1 reveals distinctive patterns in the nodulation of Papilionoid legumes by the two strains. Thus, members of several tribes appear to be responsive only to NGR234. These include the Abreae, which is monotypic, the subtribe Clitoriinae of the Phaseoleae, the subtribe Aeschynomeninae of the Aeschynomeneae, the subtribe Glycyrrhizinae of the Galegeae, the

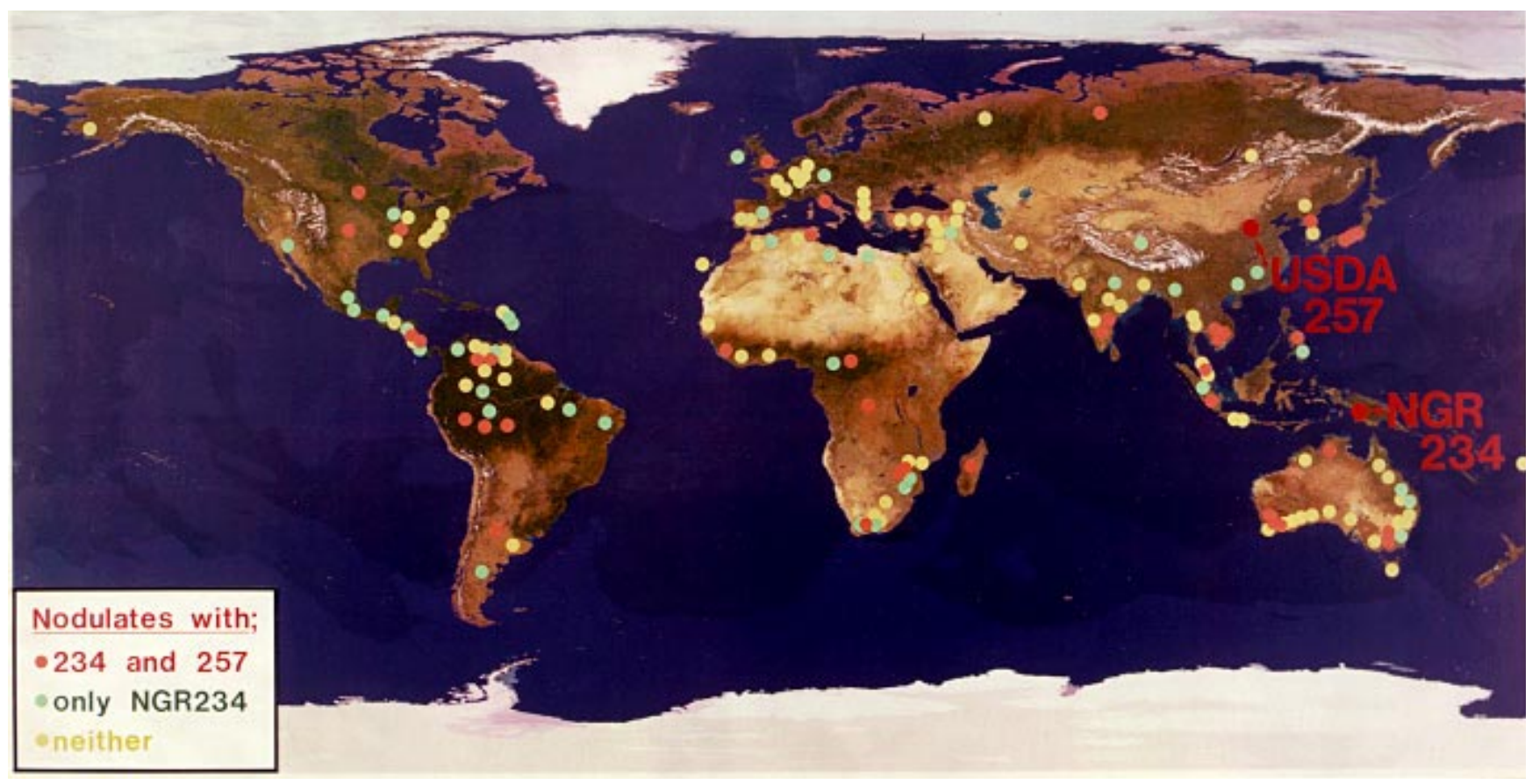

Fig. 1. Climatic map of the world showing the places of isolation of Rhizobium sp. strain NGR234 (Papua New Guinea) and R. fredii USDA257 (China). Also shown are the origins of some legume hosts and nonhosts of the two bacteria. Red spots indicate that both bacteria formed Fix ${ }^{+}$nodules on a legume originating from that region; green spots show that only NGR234 was able to nodulate the particular host; yellow spots mark origins of legumes unable to form nodules with either bacterium. Most of the data on origins of the legumes was taken from Allen and Allen (1981) and Mabberley (1987).

Table 3. Variation in the ability of three Vigna spp. to nodulate and fix nitrogen with three Bradyrhizobium and three Rhizobium strains ${ }^{\text {a }}$

\begin{tabular}{|c|c|c|c|c|c|c|}
\hline \multirow[b]{2}{*}{ Species/variety } & \multicolumn{3}{|c|}{ Bradyrhizobium japonicum } & \multicolumn{3}{|c|}{ Rhizobium spp. } \\
\hline & CB756 & USDA76 & USDA110 & NGR234 & NZP4010 & USDA257 \\
\hline V. mungo ${ }^{\mathrm{ROG}}$ & $\mathrm{Nod}^{+}$ & $\mathrm{Fix}^{+} T^{-}$ & $\mathrm{Fix}^{+}$ & $\mathrm{Fix}^{+}$ & $\mathrm{Nod}^{-}$ & $\mathrm{Fix}^{+}$ \\
\hline \multicolumn{7}{|l|}{$V$. radiata } \\
\hline King WSA & $\mathrm{Fix}^{-/^{+}}$ & $\mathrm{Nod}^{-}$ & $\mathrm{Fix}^{+}$ & $\mathrm{Fix}^{+}$ & $\mathrm{Nod}^{-}$ & $\mathrm{Fix}^{+}$ \\
\hline PUSa9173 ${ }^{\mathrm{BSU}}$ & $\mathrm{Fix}^{+}$ & $\mathrm{Fix}^{+}$ & $\mathrm{Fix}^{+}$ & $\mathrm{Fix}^{+}$ & $\mathrm{Fix}^{+}$ & $\mathrm{Fix}^{+} /^{-}$ \\
\hline RUM-1 ${ }^{\text {BSU }}$ & $\mathrm{Nod}^{-}$ & $\mathrm{Nod}^{-}$ & $\mathrm{Nod}^{-}$ & $\mathrm{Fix}^{+}$ & $\mathrm{Nod}^{-}$ & $\mathrm{Fix}^{+} \Gamma^{-}$ \\
\hline Texproust $^{\mathrm{BSU}}$ & $\mathrm{Nod}^{+}$ & $\mathrm{Fix}^{-} /^{+}$ & $\mathrm{Fix}^{+}$ & $\mathrm{Fix}^{+}$ & $\mathrm{Nod}^{-}$ & $\mathrm{Fix}^{+} I^{-}$ \\
\hline VC3890 A $\mathrm{BSU}$ & $\mathrm{Nod}^{+}$ & $\mathrm{Fix}^{-} /^{+}$ & $\mathrm{Fix}^{+}$ & $\mathrm{Fix}^{+}$ & $\mathrm{Nod}^{-}$ & $\mathrm{Fix}^{-} /^{+}$ \\
\hline VC4718 $\mathrm{A}^{\mathrm{BSU}}$ & $\mathrm{Fix}^{+}$ & Nod+ & $\mathrm{Fix}^{+}$ & $\mathrm{Fix}^{+}$ & $\mathrm{Nod}^{+}$ & $\mathrm{Fix}^{+}$ \\
\hline WM-92 $2^{\text {BSU }}$ & $\mathrm{Fix}^{+}$ & $\mathrm{Nod}^{-}$ & $\mathrm{Fix}^{+}$ & $\mathrm{Fix}^{+}$ & $\mathrm{Nod}^{+} \Gamma^{-}$ & $\mathrm{Fix}^{+}$ \\
\hline$V$. radiata subsp. sublobata ${ }^{\mathrm{BSU}}$ & $\mathrm{Nod}^{+}$ & $\mathrm{Fix}^{+}$ & $\mathrm{Fix}^{+}$ & $\mathrm{Nod}^{+}$ & $\mathrm{Nod}^{-}$ & $\mathrm{Nod}^{+}$ \\
\hline \multicolumn{7}{|l|}{ V. unguiculata } \\
\hline $\mathrm{CA} B \# 5^{\mathrm{BSU}}$ & $\mathrm{Nod}^{+}$ & $\mathrm{Fix}^{+}$ & NT & $\mathrm{Fix}^{+}$ & $\mathrm{Nod}^{-}$ & $\mathrm{Fix}^{+}$ \\
\hline PI186465 & $\mathrm{Nod}^{+}$ & $\mathrm{Fix}^{+}$ & $\mathrm{Fix}^{+}$ & $\mathrm{Fix}^{+} /-$ & $\mathrm{Nod}^{-}$ & $\mathrm{Fix}^{+}$ \\
\hline R. Caloona WSA & $\mathrm{Nod}^{+}$ & $\mathrm{Nod}^{+}$ & $\mathrm{Nod}^{+}$ & $\mathrm{Fix}^{+}$ & $\mathrm{Nod}^{-}$ & $\mathrm{Fix}^{+}$ \\
\hline TVN963 ${ }^{\mathrm{BSU}}$ & $\mathrm{Nod}^{+}$ & $\mathrm{Fix}^{+}$ & $\mathrm{Fix}^{+}$ & $\mathrm{Fix}^{+}$ & $\mathrm{Nod}^{-}$ & $\mathrm{Fix}^{+}$ \\
\hline $\mathrm{UCR} 430^{\mathrm{BSU}}$ & $\mathrm{Nod}^{+}$ & $\mathrm{Fix}^{+}$ & $\mathrm{Fix}^{+}$ & $\mathrm{Fix}^{+}$ & $\mathrm{Fix}^{-l^{+}}$ & $\mathrm{Fix}^{+}$ \\
\hline $84 \mathrm{~S}-2246-6^{\mathrm{BSU}}$ & $\mathrm{Fix}^{-}$ & Fix $^{+}$ & $\mathrm{Fix}^{+}$ & $\mathrm{Fix}^{+}$ & $\mathrm{Fix}^{-} /^{+}$ & $\mathrm{Fix}^{+}$ \\
\hline $524 \mathrm{~B}^{\mathrm{BSU}}$ & $\mathrm{Nod}^{-}$ & $\mathrm{Fix}^{+}$ & $\mathrm{Fix}^{+}$ & $\mathrm{Fix}^{+}$ & $\mathrm{Nod}^{-}$ & $\mathrm{Fix}^{+}$ \\
\hline
\end{tabular}

${ }^{a}$ Sources of seeds are as listed in the footnote to Table 1. NT = not tested. Phenotypes as in Table 2, except that in order of decreasing nitrogen-fixing efficiency they are $\mathrm{Fix}^{+}, \mathrm{Fix}^{+/-}$, and $\mathrm{Fix}^{-/+}$. Nod ${ }^{+}$nodules did not fix nitrogen. 
Podalyrieae, and the Thermopsideae. Both strains, but especially NGR234, are broadly compatible with members of the Dalbergieae, Desmodieae, Millettieae, and Phaseoleae. This is particularly evident in the Phaseoleae as NGR234 nodulates all species tested in the subtribes Diocleinae, Glycininae,
Kennediinae, and, with two exceptions (Physostigma reticulatum and Strophostyles helvola), all other species of the Phaseolinae. In the Phaseolinae, $87 \%$ of the species formed nodules that were Fix ${ }^{+}$. Perhaps adaptation to the Phaseoleae can be explained by the fact that NGR234 was isolated from

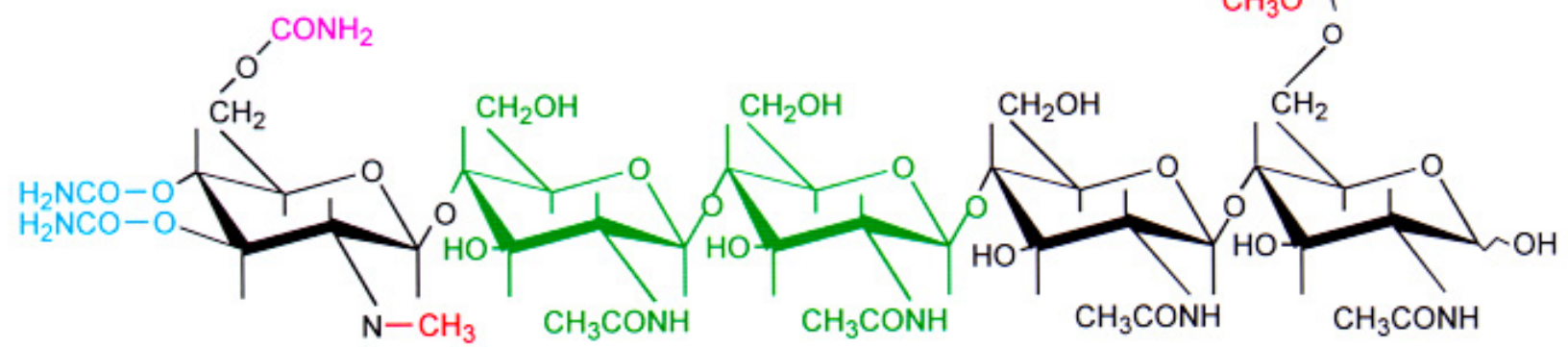<smiles>CCCCCCC=CCCCCCCCCC(N)=O</smiles>

Substituent

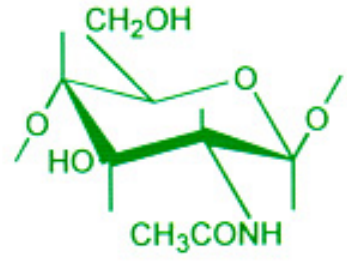

$-\mathrm{CH}_{3}$

$\mathrm{H}_{2} \mathrm{NCO}-$<smiles>NO[Na]</smiles><smiles>COC(C)=O</smiles><smiles>CS(=O)(=O)O</smiles>

$\&$

$\mathrm{H}_{2} \mathrm{NCO}-$
Genes

responsible

nodC,

nods

Intact

Mutated

nods

nodU

Mutated

nolO

Inactive

nolL

noeE

Mutated

$\mathrm{CH}_{3} \mathrm{O}-$

noel
Absent

Absent

Fig. 2. Comparison of the structures of the Nod factors produced by Rhizobium sp. strain NGR234 and R. fredii USDA257 along with the known differences in nodulation and host-specificity genes between the two organisms. Data were taken primarily from Price et al. (1992), Bec-Ferté et al. (1994), and Jabbouri et al. (1998). 
Lablab purpureus (subtribe Phaseolinae). Intriguingly, NGR234 nodulated all eight Phaseolus spp. as well as the 17 Vigna spp. tested (Table 1). In the latter case, all developed effective, nitrogen-fixing nodules. A member of the Desmodiinae, Pycnospora lutescens, is the only addition to the list of legumes known to nodulate.

Lack of nodulation in several taxonomic divisions is also significant. Included in this category are all members of two well-studied tribes: the Trifolieae, whose species are normally nodulated by $R$. meliloti and $R$. leguminosarum bv. trifolii, and the Vicieae, which are usually nodulated by $R$. leguminosarum bv. viceae (Trinick 1982). To see if exceptions to this "lack of nodulation rule" exist, the capacities of 40 different Medicago spp. were tested with $R$. meliloti as a positive control. Although $M$. cancellata and $M$. papillosa spontaneously produced nodules, neither plant responded to inoculation with NGR234 or USDA257.

Both strains failed to nodulate Cicer arietinum, the bestknown member of the sole genus constituting the Cicereae. $C$. arietinum nodulates exclusively with a group of highly specific Rhizobium strains that have affinities to $R$. leguminosarum bv. viceae (Allen and Allen 1981). Non-nodulation also occurred within certain tribes. Thus, none of the Astragalus (tribe Galegeae) species tested (normally nodulated by a group of strains now classified as $R$. haukuii), no members of the genus Onobrychis (Hedysareae) (specific for a subgroup of bradyrhizobia), nor Lotononis bainesii (Crotalarieae) (recognized as a model of acute symbiotic specificity) (Norris 1956; Allen and Allen 1981; Chen et al. 1991) nodulated with either strain.

\section{Nodulation of the nonlegume Parasponia andersonii (Ulmaceae).}

Nodulated weeds were found in tea plantations in Papua New Guinea. Initially, they were identified as Trema aspera (Trinick 1973). Further studies showed that the specimens were incorrectly identified and in fact belong to Parasponia rugosa Bl. (see Akkermans et al. 1978; Akkermans and van Dijk 1981). Later it was shown that NGR234 nodulates (albeit ineffectively) a related species, Parasponia andersonii (Trinick and Galbraith 1980). Since, for evolutionary reasons, it is important to know if USDA257 can also nodulate Parasponia spp. (see Discussion), we attempted to perform similar nodulation experiments with plants of this group. Unfortunately, we were unable to germinate any of the batches of seeds obtained, but in collaboration with G. Webster and E. C. Cocking (Plant Genetic Manipulation Group, University of Nottingham, Nottingham, UK), we tested the nodulation capacity of the two bacteria on $P$. andersonii seedlings raised in tissue culture. Both nodulated this plant (Table 1).

\section{Legume origins and nodulation capacity.}

As NGR234 was isolated in Papua New Guinea, it is often regarded as a bacterium that is specific to tropical species. To examine whether correlations between the centers of origin and the nodulation capacities of the legumes exist, those species with known origins were grouped into three classes: hosts (i.e., $\mathrm{Nod}^{+}$) to both NGR234 and USDA257; $\mathrm{Nod}^{+}$with NGR234 but $\mathrm{Nod}^{-}$with USDA257; and, Nod ${ }^{-}$with both bacteria. These data, together with the centers of origin of the legumes representing all three nodulation classes, were plotted on a map of the world that also displays regional variation in vegetation density (Fig. 1). One limitation of this analysis is that the origins of many legumes are difficult to trace, and are therefore not shown. Nevertheless, it is apparent that legumes that form nodules with both NGR234 and USDA257 can be found in most parts of the world. This includes such northern temperate species as Amorpha fruticosa, Colutea arborescens, Lespedeza bicolor, Oxytropis halleri, Robinia pseudoacacia, Ulex europaeus, and Wisteria sinensis. Equally, a number of southern temperate species form $\mathrm{Nod}^{+}$nodules with both bacteria. These include Hardenbergia comptoniana, Kennedia rubicunda, Mirbelia dilatata, Oxylobium ellipticum, and Psoralea pustulata. Finally, the tropical legumes Cajanus spp., Codariocalyx motorius, Dolichos junghuhnianus, Flemingia spp., Macrotyloma axillare, Macroptilium spp., Psophocarpus tetragonolobus, Pycnospora lutescens, Tephrosia vogelii, and Sesbania grandiflora, as well as many Vigna

Table 4. A simplified comparison of differential responses of selected legumes to inoculation with various broad-host-range rhizobia

\begin{tabular}{|c|c|c|c|c|c|c|}
\hline \multirow[b]{2}{*}{ Inoculated } & \multicolumn{6}{|c|}{ Nodulation in response to broad-host-range rhizobia ${ }^{a}$} \\
\hline & $\begin{array}{c}\text { Albizia } \\
(\text { Rhizobium })^{\dagger}\end{array}$ & $\begin{array}{c}\text { Desmodium } \\
\text { (Rhizobium })^{\dagger}\end{array}$ & $\begin{array}{c}\text { Glycine } \\
\text { USDA257 }\end{array}$ & $\begin{array}{c}\text { Lablab } \\
\text { NGR234 }\end{array}$ & $\begin{array}{c}\text { Macrotyloma } \\
\text { CB756* }\end{array}$ & $\begin{array}{c}\text { Mucuna } \\
\text { (Bradyrhizobium }) \dagger\end{array}$ \\
\hline Clitoria $\mathrm{sp}$. & + & + & - & $+/-* *$ & + & + \\
\hline Colutea arborescens & - & + & + & + & NT & - \\
\hline Desmanthus illinoensis & - & + & + & + & NT & - \\
\hline Laburnum anagyroides & + & - & - & + & NT & + \\
\hline Onobrychis vicifolia & + & + & - & - & NT & - \\
\hline Phaseolus angularis & - & - & + & + & + & - \\
\hline Pueraria phaseoloides & + & - & - & + & + & - \\
\hline Vigna unguiculata & - & - & + & + & + & + \\
\hline
\end{tabular}

${ }^{a}$ Data are from Wilson $(1939)^{\dagger}$, Diatloff and Date $(1978)^{*}$, and this work (Table 1) ${ }^{\ddagger}$. NGR234 is Fix ${ }^{+}$with Clitoria laurifolia, but Nod ${ }^{-}$with C. ternatea**. $+=$ plants were nodulated; $-=$ nodules did not form; NT = not tested.

Fig. 3. Putative relationships among tribes of the Leguminosae and the ability of Rhizobium sp. strain NGR234 and $R$. fredii USDA257 to nodulate them. "Tree" was constructed from morphological analyses of Corby et al. (1983). Tribes in yellow letters on gray background have not been tested for their nodulation capacity. Tribes in dark blue (in black, shaded boxes) are not known to nodulate. Tribes in dark-blue framed in black are Nod ${ }^{-}$with both bacteria; those in light-blue are Fix ${ }^{+}$with NGR234 but Nod ${ }^{-}$with USDA257; those marked in pink are Nod ${ }^{+}$with both bacteria; those in green are Nod ${ }^{+}$ with USDA257 and Fix ${ }^{+}$with NGR234; those in red are Fix ${ }^{+}$with both bacteria. Dotted blue arrows show absence of nodulation; dotted red arrows, loss of nodulation with NGR234/USDA257. Colors of the arrows match those of their immediate "progenitor." 


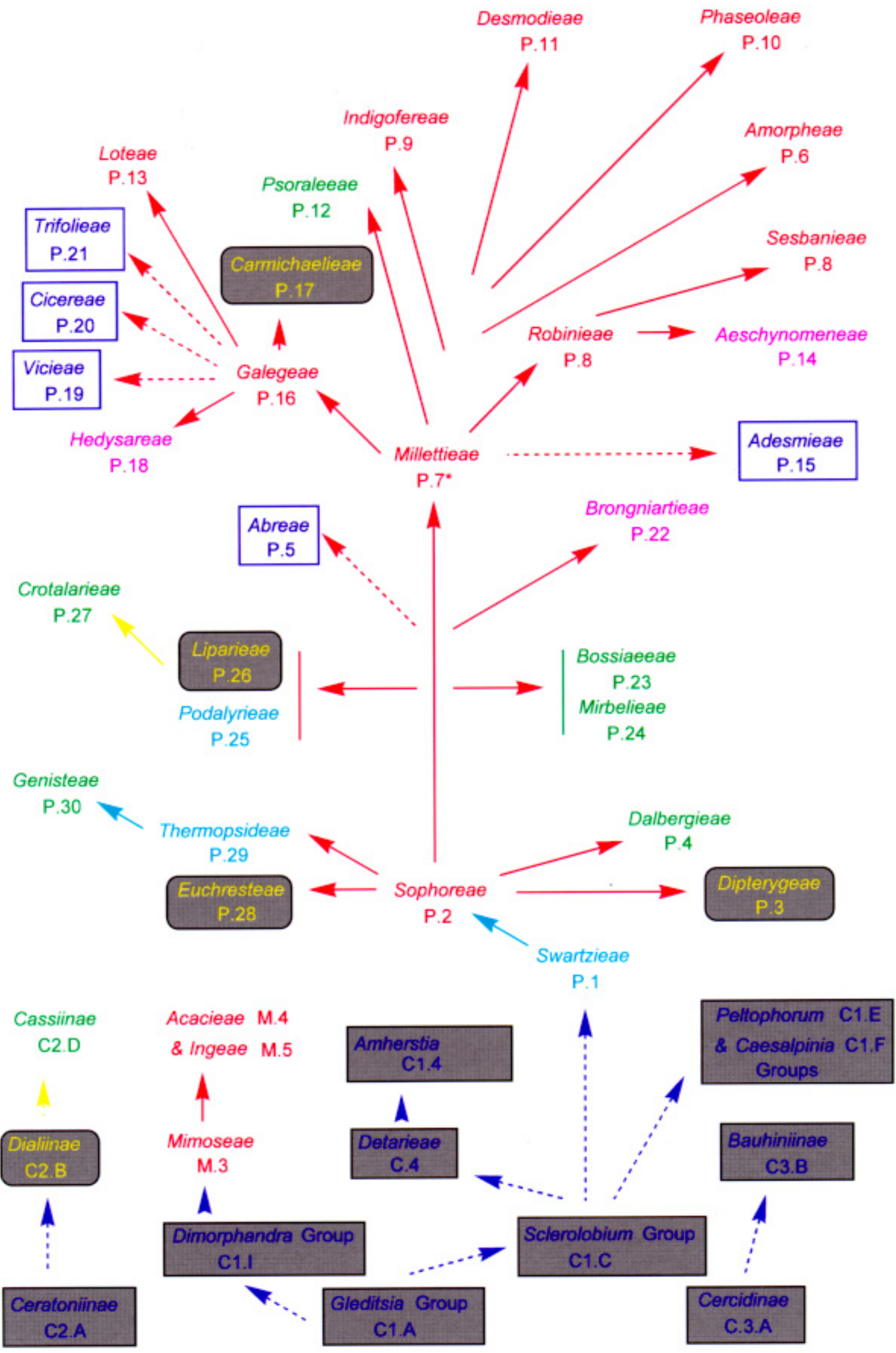


spp., form nitrogen-fixing nodules with both bacteria. Even such Mimosoid legumes as Dichrostachys cinerea (Africa, India), Albizia lebbeck (tropical Asia), Samanea saman (tropical America), and Paraserianthus falcataria are Fix ${ }^{+}$with both species. Equally, $\mathrm{Fix}^{+}$hosts are found in high-rainfall regions (Albizia spp., Codariocalyx motorius, Flemingia congesta, Indigofera tinctoria, Psophocarpus tetragonolobus, some Vigna spp., etc.) and relatively dry parts of the world (Colutea arborescens, Hardenbergia comptoniana, etc.).

\section{Legume growth habit and nodulation capacity.}

Growth habit of the host plant also seems to bear little relationship to its propensity to be nodulated by either bacterium. NGR234, for example, fixes nitrogen in association with annuals from Asia, the Americas, Australia, southern Africa, and Europe (e.g., Lablab purpureus, Phaseolus spp., Kennedia rubicundra, Psoralea spp., and Lotus spp., respectively), as well as shrubs from temperate and tropical zones (e.g., Desmanthus illinoensis, Dichrostachys cinerea, Enterolobium contortisiliquum, and Sophora spp.). Both tropical (e.g., some Acacia spp., Albizia lebbeck, Leucaena leucocephala,
Mundulea sericea, Samanea saman, and Xeroderris stuhlmannii), and temperate trees (Erythrina crista-galli, Colutea arborescens, Hesperolaburnum platycarpum, Sophora davidii, and Robinia pseudoacacia) are effectively nodulated by NGR234 (Table 1). Species as ecologically diverse as Lotus corniculatus, a forage legume known for its heat and drought tolerance, and Neptunia oleracea, a tropical aquatic legume, are also nodulated by NGR234.

\section{Comparison of the USDA257 and NGR234 host ranges.}

Perhaps the most striking correlation observed here is that all 135 legume species nodulated by USDA257 are also host to NGR234. Thus, with respect to nodulation, the host range of USDA257 is a subset nested entirely within that of NGR234. In the 135 combinations in which both strains nodulated the same plant, $30 \%$ of all the nodules were $\mathrm{Nod}^{+} \mathrm{Fix}^{-}$with both bacteria, $46 \%$ were $\mathrm{Fix}^{+}$with both bacteria, while NGR234 produced Fix ${ }^{+}$nodules on $64 \%$ of these plants. In other words, NGR234 also has a symbiotic advantage over USDA257 in terms of nitrogen fixation. Apios americana, G. max, and G. soja (wild soybean) constitute the

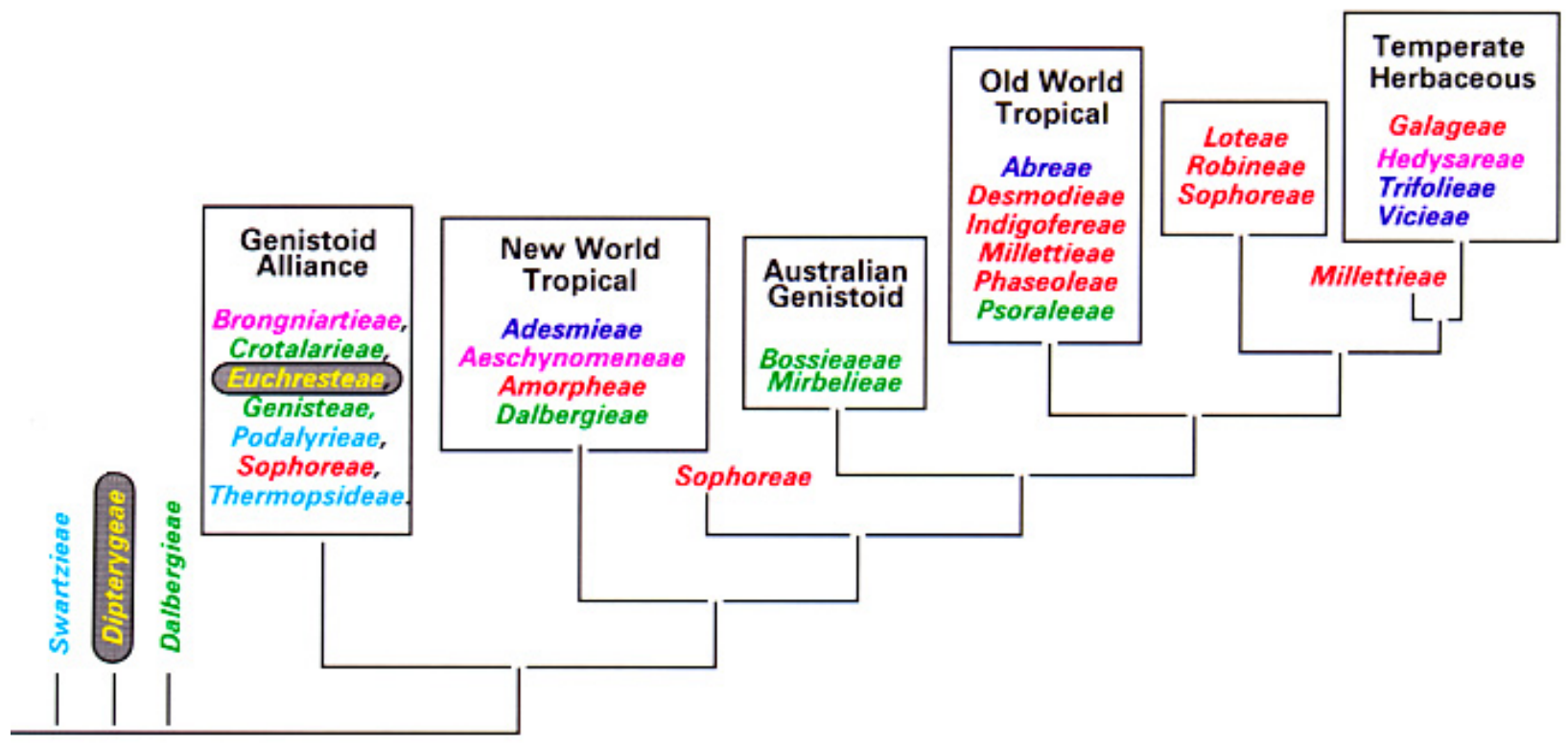

Fig. 4. Tribal relationships in the Papilionoideae, inferred from sequences of the chloroplast gene ribulose-1,5-bisphosphate carboxylase/oxygenase $(r b c \mathrm{~L})$ strict consensus tree (Doyle et al. 1997). Color coding for the capacity of the various tribes to nodulate with NGR234/USDA257 is the same as shown in Figure 3.

Table 5. Bradyrhizobiu/Rhizobium strains used in this study

\begin{tabular}{|c|c|c|c|}
\hline Strain & Host of isolation & Characteristics $^{\mathrm{a}}$ & Reference \\
\hline B. elkanii USDA76 & Glycine $\max$ & $\begin{array}{l}\text { Isolated from USDA74 by plant passage. Sero- } \\
\text { group } 76 \text { type strain }\end{array}$ & Kuykendall et al. 1992 \\
\hline B. japonicum $\mathrm{CB} 756$ & Macrotyloma africanum & Broad-host-range inoculant. Ap ${ }^{\mathrm{r}}, \mathrm{Rif}^{\mathrm{r}}$ & Norris 1956; Diatloff and Date 1978 \\
\hline B. japonicum USDA110 & G. $\max$ & $\begin{array}{l}\text { Genetically best-characterized B. japonicum spe- } \\
\text { cies. Sp } \mathrm{s}^{\mathrm{r}}\end{array}$ & Kuykendall and Elkan 1976 \\
\hline Rhizobium sp. strain NGR234 & Lablab purpureus & Broad host range. Rif $^{\mathrm{r}}$ & Trinick 1980 \\
\hline R. loti NZP4010 & Lotus divaricatus & $\begin{array}{l}=\text { NZP2037 cured of its plasmid, broad host range. } \\
\text { Rif }^{\mathrm{T}}, \mathrm{Sm}^{\mathrm{r}}\end{array}$ & Chua et al. 1985; Lewin et al. 1987b \\
\hline R. fredii USDA257S1 & Glycine soja & $\begin{array}{l}\mathrm{Km}^{\mathrm{r}} \text { derivative of wild-type USDA257. Carries a } \\
\text { silent } \mathrm{Tn} 5 \text { insertion in the Sym plasmid }\end{array}$ & Heron et al. 1989 \\
\hline R. meliloti RCR2011 & Medicago sativa & $=\mathrm{SU} 47 \cdot \mathrm{Sm}^{\mathrm{r}}, \mathrm{Tc}^{\mathrm{r}}$ & Rosenberg et al. 1981 \\
\hline
\end{tabular}

a Antibiotics used: $\mathrm{Ap}=$ ampicillin; Rif = rifampicin; $\mathrm{Sm}=$ streptomycin; and Tc = tetracycline. 
Table 1. Host range of Rhizobium sp. strain NGR234 and R. fredii USDA257. Classification of the Leguminosae follows that of Corby et al. (1983) and Polhill (1994). C, M, and P are abbreviations for the subfamily names CAESALPINIOIDEAE, MIMOSOIDEAE, and PAPILIONOIDEAE, respectively, while the numbers correspond to the tribes and genera of Polhill (1994). N denotes nodulation (but not nitrogen fixation), F that the nodules were effective, and a dash the absence of nodules. Shaded species have never been observed to nodulate, while the boxed species is the first report of nodulation. Legumes printed in green have indeterminate nodules, those in blue, determinate nodules, names in pink are species possessing Aeschynomenoid nodules, while the information available for those printed in black is either inconsistent or insufficient to determine the nodule type. Names in brackets are obsolete.

Index Abrus P5.1; Acacia M4.1; Acrocarpus C1.3; Adenanthera M3.9; Adenolobus C3.2, Adesmia P15.1; Aeschynomene P14.9; Albizia M5.3; Alysicarpus P11.18; Amorpha P6.4; Amphicarpaea P10.44; Anadenanthera M3.26; Anagyris P29.3; Anthyllis P13.2; Aotus P24.21; Apios P10.7; Arachis P14.26; Astragalus P16.15; Ateleia P1.13; Baptisia P29.5; Bauhinia C3.4; Bituminaria P12.2; Bolusanthus P2.37; Bossiaea P23.6; Brachysema P24.16; Burkea C1.45; (Burtonia) P24.1; Caesalpinia CI.24; Cajanus P10.73; Calicotome P30.17, Calliandra M5.9; Calopogonium P10.45; Canavalia P10.21; Caragana P16.11; Cassia C2.16; Castanospermum P2.12; Centrosema P10.14; Ceratonia C2.1; Cercis C3.1; Chamaecrista C2.18; Chamaecytisus P30.15; Chorizema P24.10; Christia P11.17; Cicer P20.1; Cladrastis P2.41; Clianthus P16.1; Clitoria P10.16; Codariocalyx P11.10; Coluted P16.5; Coronilla P13.11; Coursetia P8.8; Cratylia P10.25; Crotalaria P27.7; Cyamopsis P9.6; Cynometra C4.1; Cytisus P30.15; Dalbergia P4.3; Dalea P6.8; Daviesia P24.4; Delonix C1.22; Dendrolobium P11.6; Desmanthus M3.35; Desmodium P11.9; Derris P7.14; Dicerma P11.8; Dichrostachys M3.32; Dillwynia P24.25; Dinizia M3.1; Dioclea P10.18; Dolichos P10.64; Dorycnium P13.6; Dunbaria P10.74; Dysolobium P10.50; Echinospartum P30.23; Enterolobium M5.7; Eriosema P10.81; Erythrina P10.1; Faidherbia M5.8; Flemingia P10.77; Galactia P10.27; Galega P16.20; Gastrolobium P24.13; Genista P30.22; Gleditsia C1.2; Gliricidia P8.6; Glycine P10.35; Glycyrrhiza P16.22; Gompholobium P24.1; Goodia P23.5; Gueldenstaedtia P16.19; Gymnocladus C1.1; Halimodendron P16.10, Hardenbergia P10.48; Hardwickia C4.34; Hedysarum P18.2; Hesperolaburnum P30.10; Hippocrepi. P13.13; Hoffmannseggia C1.34; Hovea P22.4; Hymenocarpos P13.4; Indigofera P9.7; Inga M5.1; Isotropis P24.6; Jacksonia P24.8; Kennedia P10.47; Kummerowia P11.25; Labichea C2.19; Lablab P10.61; Laburnum P30.9; Lathyrus P19.2; Lembotropis P30.16; Lens P19.3; Lespedeza P11.24; Leucaena M3.30; Lotononis P27.9; Lotus P13.7; Lupinus P30.8; Maackia P2.40; Macroptilium P10.71; Macrotyloma P10.65; Medicago P21.5; Melilotus P21.3; Millettia P7.23; Mimosa M3.27; Mirbelia P24.11; Mucuna P10.3; Mundulea P7.25; Nemcia P24.15 Oxylobium P24.9; Neonotonia P10.40; Neptunia M3.36; Onobrychis P18.6; Ononis P21.1; Ornithopus P13.15; Otoptera P10.56; Oxylobium P24.9; Oxytropis P16.17; Pachecoa P14.23, Pachyrhizus P10.46; Paracalyx P10.82; Paraserianthes M5.14; Parkia M1.2; Parkinsonia C1.20; Peltophorum C1.15; Petalostylis C2.20; Phaseolus P10.72; Phyllodium P11.7; Piliostigma C3.4; Piptadenia M3.22; Piptanthus P29.2; Pisum P19.4; Pithecellobium M5.11; Pongamia P7.34, Prosopis M3.16; Pseudarthria P11.11; Psophocarpus P10.51; Psoralea P12.9; Pterocarpus P4.17; Pueraria P10.32; Pultenaea P24.23; Pycnospora P11.12; Retama P30.21; Rhynchosia P10.80; Robinia P8.7; Samanea M5.5; Schizolobium C1.17; Schotia C4.8; Scorpiurus P13.14; Securigera P13.12; Senna C2.17; Sesbania P8.1; Sophora P2.45; Spartium P30.19; Strophostyles P10.70; Stryphnodendron M3.20; Styphnolobium P2.44; Stylosanthes P14.25; Sutherlandia P16.3; Swainsona P16.2; Tadehagi P11.13; Tamarindus C4.74; Templetonia P22.4; Tephrosic P7.40; Teramnus P10.36; Teyleria P10.39; Thermopsis P29.4; Tipuana P4.13; Trifolium P21.6; Trigonella P21.4; Ulex P32.25; Vicia P19.1; Vigna P10.66; Viminaria P24.3; Virgilia P25.3; Wisteria P7.43; Xeroderris P7.44; Zornia P14.21

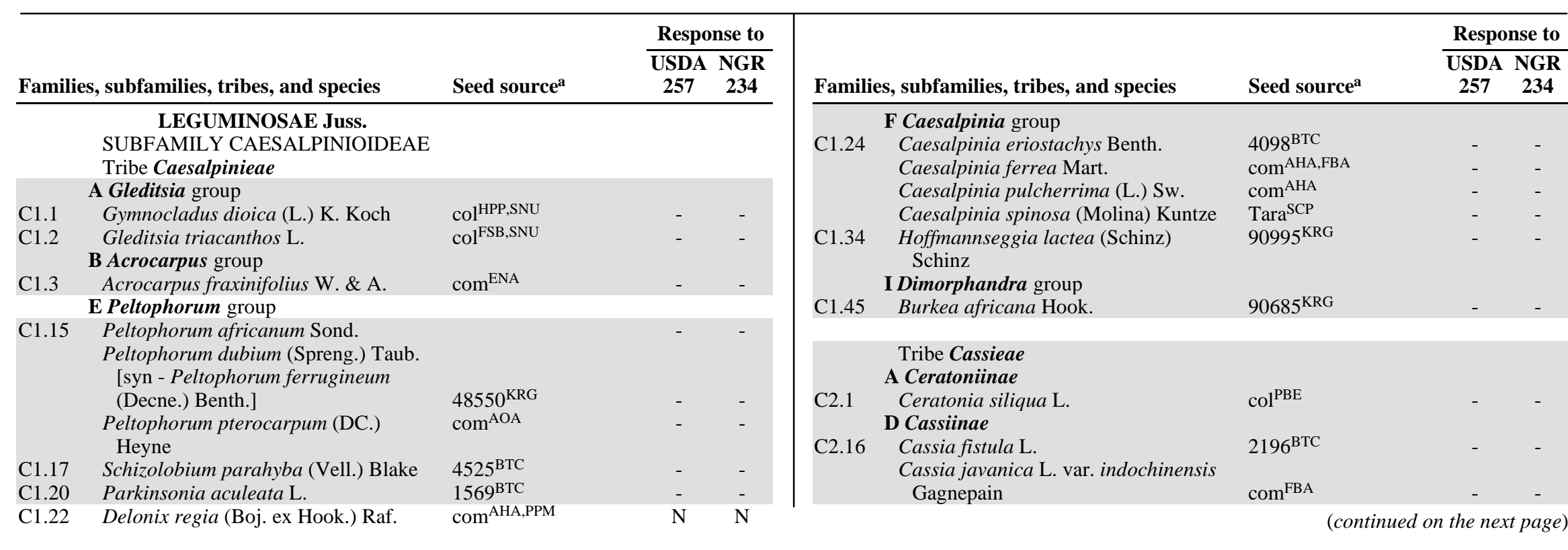




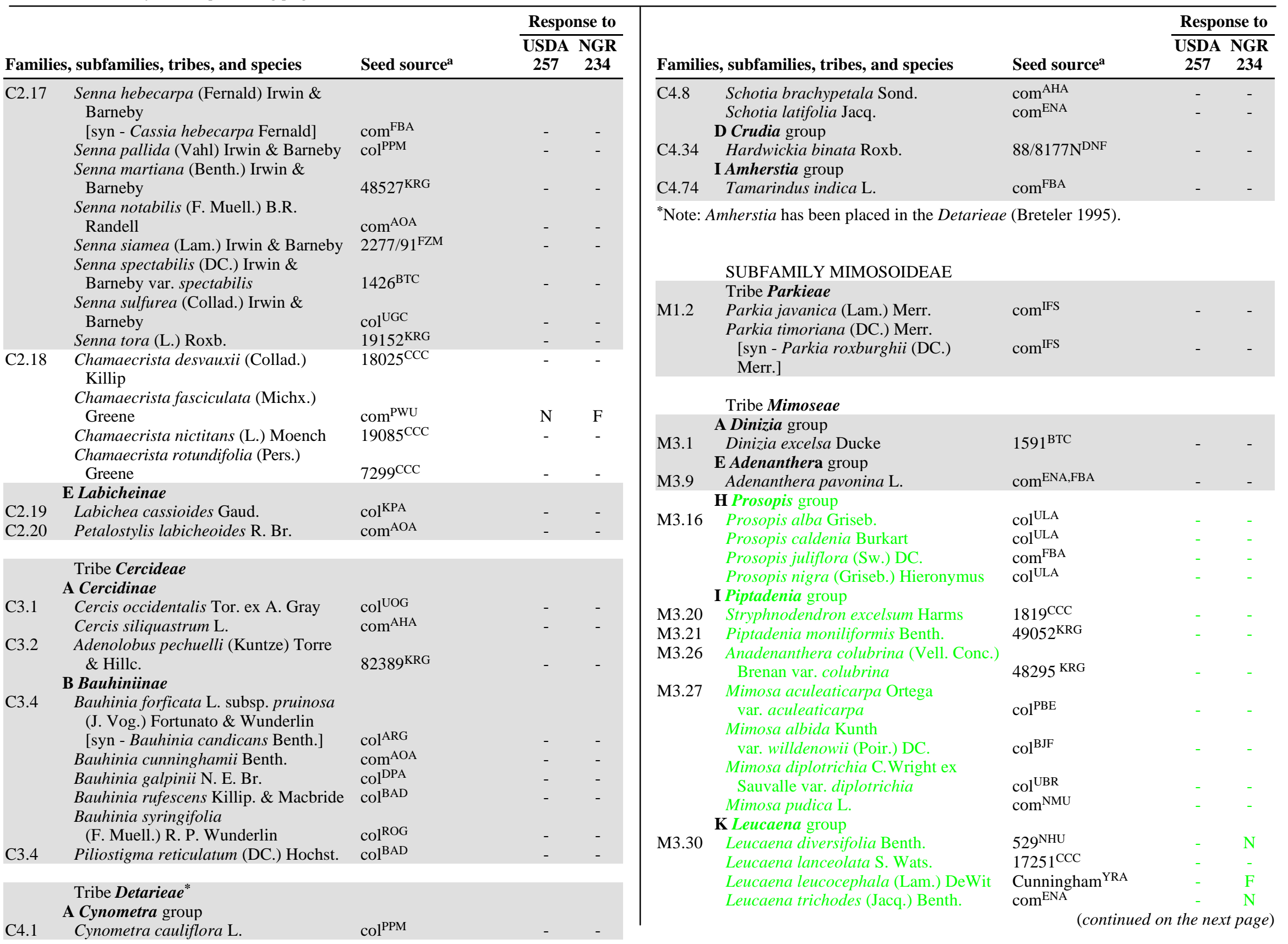




\begin{tabular}{|c|c|c|c|c|c|c|c|c|c|}
\hline & & & Resp & onse to & & & & Respo & nse to \\
\hline Familie & es, subfamilies, tribes, and species & Seed source $^{a}$ & $\begin{array}{c}\overline{\text { USDA }} \\
\mathbf{2 5 7}\end{array}$ & $\begin{array}{c}\text { NGR } \\
234\end{array}$ & Famili & es, subfamilies, tribes, and species & Seed source ${ }^{a}$ & $\begin{array}{c}\text { USDA } \\
257\end{array}$ & $\begin{array}{c}\text { NGR } \\
234\end{array}$ \\
\hline & L Dichrostachys group & & & & & Standley var. anomala (Kunth) & & & \\
\hline M3.32 & Dichrostachys cinerea (L.) W. \& A. & $19093^{\mathrm{CCC}}$ & $\mathrm{F}$ & $\mathrm{F}$ & & Barneby ${ }^{\S}$ syn - Calliandra & & & \\
\hline M3.35 & Desmanthus illinoensis & & & & & grandiflora Benth]. & $\mathrm{col}^{\mathrm{JTF}}$ & - & $\mathrm{N}$ \\
\hline & (Michx.) MacMill. ex Robinson & $\mathrm{col}^{\mathrm{PCU}}$ & $\mathrm{N}$ & $\mathrm{F}$ & & Calliandra houstoniana (Miller) & & & \\
\hline & Desmanthus virgatus (L.) Willd. & $\operatorname{Marc}^{\mathrm{ASA}}$ & - & $\mathrm{F}$ & & Standley var. calothyrsus & & & \\
\hline M3.36 & Neptunia gracilis Benth. & & & & & $(\text { Meissner \& Barneby })^{\S}$ & & & \\
\hline & var. major (Benth.) Windler & $\mathrm{col}^{\mathrm{KPA}}$ & - & - & & [syn - Calliandra calothyrsus & HA,IFS & & \\
\hline & Neptunia natans (L.) Druce & & & & & $\begin{array}{l}\text { Meissner] } \\
\text { Pithecellobium dulce (Roxb.) Benth. }\end{array}$ & $\operatorname{com}^{\mathrm{IFS}}$ & - & - \\
\hline & Tribe Acacieae $^{\mathrm{qI}}$ & & & & M5.14 & Paraserianthes falcataria (L.) Nielsen & $\mathrm{col}^{\mathrm{AHA}, \mathrm{NHU}}$ & $\mathrm{F}$ & $\mathrm{F}$ \\
\hline M4.1 & A subgen. Acacia & & & & & Paraserianthes lophantha (Willd.) & & & \\
\hline & Acacia farnesiana (L.) Willd. & $\operatorname{com}^{\mathrm{AHA}}$ & - & $\mathrm{N}$ & & Nielsen & $\operatorname{com}^{\mathrm{AHA}}$ & - & - \\
\hline & Acacia karroo Hayne & $\operatorname{col}^{\mathrm{BJF}}$ & - & & $\S_{\text {see } \mathrm{Ba}}$ & rneby (1998) & & & \\
\hline & B subgen. Aculeiferum & & & & & & & & \\
\hline & Acacia aroma Gillis ex H.\&A. & $\mathrm{col}^{\mathrm{ULA}}$ & - & - & & & & & \\
\hline & Acacia ataxacantha DC. & $\mathrm{col}^{\mathrm{BAD}}$ & - & - & & SUBFAMILY PAPILIONOIDEAE & & & \\
\hline & Acacia bonariensis Gillis ex H. \& A. & $\mathrm{col}^{\mathrm{ULA}}$ & - & $\mathrm{N}$ & & Tribe Swartzieae & & & \\
\hline & & $\mathrm{col}^{\text {ULA }}$ & - & $\mathrm{N}$ & & D Ateleia group $¥$ & & & \\
\hline & Acacia polyacantha Willd. & $\begin{array}{l}\mathrm{col}^{\mathrm{ODS}} \\
273^{\mathrm{BAD}}\end{array}$ & - & - & $\mathrm{P} 1.13$ & Ateleia ovata Mohlenbr. & $7362^{\mathrm{CCC}}$ & - & $\mathrm{F}$ \\
\hline & & 13 & & & ${ }^{¥}$ see Po & lhill (1994) & & & \\
\hline & Acacia auriculiformis A. Cunn. & $365^{\mathrm{NHU}}$ & $\mathrm{N}$ & $\mathrm{F}$ & & & & & \\
\hline & Acacia cyanophylla Lindl. & $\mathrm{col}^{\mathrm{FNC}}$ & $\mathrm{N}$ & $\mathrm{N}$ & & Tribe Sophoreae & & & \\
\hline & Acacia mangium Willd. & $\operatorname{com}^{\mathrm{AHA}}$ & $\mathrm{N}$ & $\mathrm{N}$ & & B Angylocalyx group & & & \\
\hline & Acacia mearnsii De Wild. & $\mathrm{col}^{\mathrm{NHU}}$ & $\mathrm{N}$ & $\mathrm{N}$ & $\mathrm{P} 2.12$ & Castanospermum australe A. Cunn. & $\operatorname{com}^{\mathrm{AHA}, \mathrm{FBA}}$ & - & - \\
\hline & Acacia pendula A. Cunn. ex. G. Don & $\operatorname{com}^{\mathrm{FBA}}$ & - & $\mathrm{F}$ & & F Sophora group & & & \\
\hline & Acacia retinodes Schltdl. & $\operatorname{com}^{\mathrm{AOA}}$ & $\mathrm{N}$ & $\mathrm{F}$ & $\mathrm{P} 2.37$ & Bolusanthus speciosus (Bolus) Harms & $\operatorname{com}^{\text {ENA }}$ & - & $\mathrm{N}$ \\
\hline & Acacia saligna (Labill.) Wendl. & $\operatorname{com}^{\mathrm{AHA}}$ & $\mathrm{N}$ & $\mathrm{F}$ & $\mathrm{P} 2.40$ & Maackia amurensis Rupr. \& Maxim. & $\mathrm{col}^{\mathrm{SNU}}$ & - & - \\
\hline IDivisio & on of the tribe Acacieae into subgenera fo & llows the recor & tions $\mathrm{c}-\mathrm{c}$ & Vassal & P2.41 & Cladrastis lutea (Michx. f.) Koch & $\mathrm{col}^{\mathrm{SNU}}$ & - & - \\
\hline (1981). & & & & & P2.44 & Styphnolobium japonicum Schott & $\mathrm{col}^{\mathrm{JMF}}$ & - & - \\
\hline & & & & & P2.45 & Sophora davidii (Franch.) Pavol. & $\mathrm{col}^{\mathrm{CGC}}$ & $\mathrm{F}$ & $\mathrm{F}$ \\
\hline & Tribe Ingeae & & & & & Sophora microphylla Aiton & $\mathrm{col}^{\mathrm{Cu}}$ & - & - \\
\hline M5.1 & Inga mortoniana J. León. & $\operatorname{com}^{\mathrm{FSB}}$ & $\mathrm{F}$ & $\mathrm{F}$ & & Sophora tetraptera J. Mill. & $\operatorname{com}^{\mathrm{CNA}}$ & - & - \\
\hline M5.3 & Albizia julibrissin Durazz. & $\mathrm{col}^{\mathrm{CGC}}$ & $\mathrm{N}$ & $\mathrm{N}$ & & Sophora tomentosa $\mathrm{L}$. & $\operatorname{com}^{\mathrm{AHA}}$ & $\mathrm{F}$ & $\mathrm{F}$ \\
\hline & Albizia lebbeck (L.) Benth. & $14959^{\mathrm{ACA}}$ & $\mathrm{F}$ & $\mathrm{F}$ & & Sophora velutina Lindl. & $\mathrm{col}^{\mathrm{NHZ}}$ & $\mathrm{F}$ & $\mathrm{F}$ \\
\hline & Albizia procera (Roxb.) Benth. & $\operatorname{com}^{\mathrm{FBA}}$ & $\mathrm{F}$ & $\mathrm{F}$ & & Tribe Dalbergieae & & & \\
\hline & Albizia saponaria (Lour.) Miq. & $\mathrm{col}^{\mathrm{UBR}}$ & $\mathrm{N}$ & $\mathrm{N}$ & & B Dalbergia group & & & \\
\hline M5.5 & Samanea saman (Jacq.) Merr. & $21812^{\mathrm{CCC}}$ & $\mathrm{F}$ & $\mathrm{F}$ & $\mathrm{P} 4.5$ & Dalbergia martinii F. White & $\mathrm{col}^{\mathrm{NHZ}}$ & $\mathrm{N}$ & $\mathrm{N}$ \\
\hline M5.7 & Enterolobium contortisiliquum & & & & & Dalbergia retusa Hemsl. & $2478^{\mathrm{BTC}}$ & - & $\mathrm{F}$ \\
\hline & ell.) Morong & $\mathrm{col}^{\mathrm{ULA}}$ & $\mathrm{N}$ & $\mathrm{F}$ & P4.13 & Tipuana tipu (Benth.) Kuntze & $\mathrm{col}^{\mathrm{AOA}, \mathrm{UGC}}$ & $\mathrm{N}$ & $\mathrm{N}$ \\
\hline$M$ & Enterolobium timbouva Mart. & $\operatorname{com}^{\mathrm{ENA}}$ & $\overline{\mathrm{F}}$ & $\mathrm{F}$ & P4.17 & Pterocarpus lucens Guill. \& Perr. & $\mathrm{col}^{\mathrm{BAD}}$ & $\mathrm{N}$ & $\mathrm{F}$ \\
\hline MIS.8 & 1.) & $2067 / 89^{\mathrm{FZM}}$ & $\mathrm{F}$ & & & & & & \\
\hline M5.9 & Calliandra houstoniana (Miller) & & & & & & & & \\
\hline
\end{tabular}




\begin{tabular}{|c|c|c|c|c|c|c|c|c|c|}
\hline & & & Resp & onse to & & & & Respo & nse to \\
\hline & & & $\overline{\text { USDA }}$ & $\overline{\text { NGR }}$ & & & & $\overline{\text { USDA }}$ & NGR \\
\hline Famili & s, subfamilies, tribes, and species & Seed source $^{a}$ & 257 & 234 & Familie & s, subfamilies, tribes, and species & Seed source $^{a}$ & 257 & 234 \\
\hline & Tribe Abreae & & & & $\overline{\mathrm{P} 8.8}$ & Coursetia caribaea (Jacq.) M. Lavin & $9392^{\mathrm{CCC}}$ & - & - \\
\hline P5.1 & Abrus precatorius $\mathrm{L}$. & $\mathrm{col}^{\mathrm{ILF}, \mathrm{IFS}}$ & - & - & & Tribe Indigofereae & & & \\
\hline & Tribe Amorpheae & & & & P9.6 & Cyamopsis tetragonoloba (L.) Taub. & Essex ${ }^{\mathrm{PCU}}$ & & - \\
\hline P6.4 & Amorpha fruticosa $\mathrm{L}$. & $\mathrm{col}^{\mathrm{JTF}, \mathrm{PBE}}$ & $\mathrm{F}$ & $\mathrm{F}$ & P9.7 & Indigofera arrecta Hochst. ex A. Rich. & $18661^{\mathrm{ASA}}$ & F & $\mathrm{F}$ \\
\hline P6.8 & Dalea candida Willd. & $\mathrm{col}^{\mathrm{BMU}}$ & - & $\mathrm{F}$ & & Indifogera australis Willd. & $\mathrm{col}^{\mathrm{WBA}}$ & $\mathrm{N}$ & $\mathrm{N}$ \\
\hline & Dalea purpurea Ventenat & $\operatorname{com}^{\mathrm{PWU}}$ & $\mathrm{F}$ & $\mathrm{F}$ & & Indigofera glandulosa Willd. & $\mathrm{col}^{\mathrm{UGC}}$ & - & - \\
\hline & & & & & & Indigofera jamaicensis Spreng. & $9319^{\mathrm{CCC}}$ & - & $\mathrm{F}$ \\
\hline & Tribe Millettieae $\left.\right|^{\dagger}$ & & & & & Indigofera lespedezioides HBK & $9048^{\mathrm{CCC}}$ & - & - \\
\hline P7.14 & Derris robusta (DC.) Benth. & $\operatorname{com}^{\mathrm{IFS}}$ & - & - & & Indigofera tinctoria $\mathrm{L}$. & $\operatorname{com}^{\mathrm{IFS}}$ & $\mathrm{F}$ & $\mathrm{F}$ \\
\hline P7.25 & Mundulea sericea (Willd.) A. Chev. & $84176^{\mathrm{KRG}}$ & $\mathrm{F}$ & $\mathrm{F}$ & & & & & \\
\hline P7.23 & Millettia megasperma Benth. & $\operatorname{com}^{\mathrm{AHA}, \mathrm{SDS}}$ & $\mathrm{N}$ & $\mathrm{N}$ & & Tribe Phaseoleae & & & \\
\hline P7.34 & Pongamia pinnata (L.) Pierre & $\mathrm{com}^{\mathrm{AOA}}$ & - & - & & A Erythrininae & & & \\
\hline P7.40 & Tephrosia cinerea (L.) Pers. & $9459^{\mathrm{CCC}}$ & - & $\mathrm{F}$ & P10.1 & Erythrina abyssinica DC. & $\mathrm{col}^{\mathrm{NHZ}}$ & - & $\mathrm{F}$ \\
\hline & Tephrosia rosea F. Muell. ex Benth. & $\operatorname{com}^{\mathrm{AOA}}$ & - & $\mathrm{F}$ & & Erythrina costaricensis M. Micheli & $4153^{\mathrm{BTC}}$ & $\mathrm{F}$ & $\mathrm{F}$ \\
\hline & Tephrosia sessiliflora (Poir.) Hassl. & $9155^{\mathrm{CCC}}$ & - & $\mathrm{F}$ & & Erythrina crista-galli $\mathrm{L}$. & $\mathrm{col}^{\mathrm{PBE}, \mathrm{ULA}}$ & - & $\mathrm{F}$ \\
\hline & Tephrosia vogelii Hook. f. & $387879^{\mathrm{SGU}}$ & $\mathrm{F}$ & $\mathrm{F}$ & & Erythrina fusca Lour. & $21601^{\mathrm{CCC}}$ & - & $\mathrm{F}$ \\
\hline P7.43 & Wisteria frutescens (L.) Poir. & $\operatorname{com}^{\text {ENA }}$ & - & $\mathrm{N}$ & & Erythrina poeppigiana (Walpers) & & & \\
\hline & Wisteria sinensis (Sims) Sw. & $\mathrm{col}^{\mathrm{UOG}}$ & $\mathrm{N}$ & $\mathrm{N}$ & & O.F. Cook & $4520^{\mathrm{BTC}}$ & - & - \\
\hline P7.44 & Xeroderris stuhlmannii (Taub.) Mend. & & & & & Erythrina variegata $\mathrm{L}$. & $\mathrm{com}^{\mathrm{FBA}, \mathrm{TSU}}$ & - & $\mathrm{N}$ \\
\hline & $\&$ Sousa & $\mathrm{col}^{\mathrm{NHZ}}$ & $\mathrm{F}$ & F & & Erythrina vespertilio Benth. & $\operatorname{com}^{\mathrm{FBA}}$ & - & $\mathrm{F}$ \\
\hline$\dagger$ Num & $\mathrm{n}$ this tribe follow the alphabetical s & em of Corby et al. & 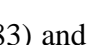 & & P10.3 & Mucuna pruriens (L.) DC. & $\operatorname{com}^{\mathrm{GKM}, \mathrm{IFS}}$ & - & - \\
\hline taxon & In this tinde lonow & & & & P10.7 & $\begin{array}{l}\text { Apios americana Medik. } \\
\text { C Clitoriinae }\end{array}$ & $\mathrm{col}^{\mathrm{HBU}}$ & $\mathrm{F}$ & $\mathrm{N}$ \\
\hline & Tribe Robinieae & & & & P10.14 & Centrosema pubescens Benth. & $\operatorname{com}^{\mathrm{GKM}}$, IFS & - & $\mathrm{N}$ \\
\hline & A Sesbania group & & & & P10.16 & Clitoria laurifolia Poir. & $17366^{\mathrm{CCC}}$ & - & $\mathrm{F}$ \\
\hline P8.1 & Sesbania sesban (L.) Merr. & & & & & Clitoria ternatea $\mathrm{L}$. & $\operatorname{com}^{\mathrm{TJU}}$ & - & - \\
\hline & [syn - Sesbania aegyptiaca Poir.] & $\operatorname{com}^{\mathrm{IFS}}, 2296 / 91^{\mathrm{FZM}}$ & - & - & & D Diocleinae & & & \\
\hline & Sesbania bispinosa (Jacq.) W.F. Wight & $\mathrm{col}^{\mathrm{PPM}}$ & $\mathrm{N}$ & $\mathrm{N}$ & P10.18 & Dioclea guianensis Benth. & 7351 & $\mathrm{~N}$ & $\mathrm{~N}$ \\
\hline & Sesbania cannabina (Retz.) Pers. & $\operatorname{com}^{\mathrm{AOA}}$ & $\mathrm{N}$ & $\mathrm{N}$ & & Dioclea sericea $\mathrm{HBK}$ & 8434 & $\mathrm{~N}$ & $\mathrm{~N}$ \\
\hline & Sesbania formosa (F.Muell.) N. Burb. & $\operatorname{com}^{\mathrm{AHA}}$ & - & - & & Dioclea virgata (L.C. Rich.) Amshoff & $18124^{\mathrm{CCC}}$ & - & $\mathrm{N}$ \\
\hline & Sesbania grandiflora (L.) Pers. & $\operatorname{com}^{\mathrm{AHA}}$ & $\mathrm{F}$ & F & P10.21 & Canavalia ensiformis (L.) DC. & $\operatorname{col}^{\mathrm{PC}}$ & $\mathrm{N}$ & $\mathrm{N}$ \\
\hline & Sesbania herbacea (Mill.) R. & $\operatorname{com}^{\mathrm{SSU}}$ & - & - & & $\begin{array}{l}\text { Canavalla rosea (Sw.) DC. } \\
\text { [syn - Canavalia maritima Thouars] }\end{array}$ & $\operatorname{com}^{\mathrm{AHA}, \mathrm{AOA}}$ & $\mathrm{N}$ & $\mathrm{F}$ \\
\hline & $\begin{array}{l}\text { Mesbania punicea (Cav.) Benth. } \\
\text { Sent }\end{array}$ & $\mathrm{col}^{\mathrm{ULA}}$ & - & - & $\mathrm{P} 10.25$ & Cratylia argentea (Desv.) O. Kuntze & $18957^{\mathrm{CCC}}$ & - & $\mathrm{N}$ \\
\hline & Sesbania rostrata Brem. \& Oberm. & $\mathrm{col}$ ODS & - & $\mathrm{N}$ & P10.27 & Galactia jussiaeana Kunth & $8805^{\mathrm{CCC}}$ & $\mathrm{N}$ & $\mathrm{F}$ \\
\hline & 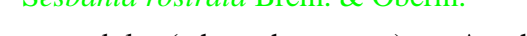 & & & & & Galactia latisiliqua Desv. & $923^{\mathrm{CCC}}$ & $\mathrm{N}$ & $\mathrm{N}$ \\
\hline FNote - & $\begin{array}{l}\text { stem nodules (where they occur) are Aesch } \\
\text { C Gliricidia group }\end{array}$ & ynomenoid. & & & & $\begin{array}{l}\text { Galactia striata (Jacq.) Urb. } \\
\text { E Glycininae }\end{array}$ & $464^{\mathrm{ASA}}$ & - & $\mathrm{N}$ \\
\hline P8.6 & Gliricidia maculata HBK & $\operatorname{com}^{\mathrm{IFS}}$ & - & - & P10.32 & Pueraria lobata (Willd.) Ohwi & $\mathrm{col}^{\mathrm{PTJ}}$ & $\mathrm{N}$ & $\mathrm{N}$ \\
\hline & D Robinia group & & & & & Pueraria phaseoloides (Roxb.) Benth. & $\mathrm{col}^{\mathrm{IFS}}$ & - & $\mathrm{N}$ \\
\hline P8.7 & Robinia hispida L. var. fertilis (Ashe) & & & & P10.35 & Glycine canescens Herman & $\mathrm{col}^{\mathrm{PCU}}$ & - & $\mathrm{F}$ \\
\hline & R.T. Clausen [syn - Robinia fertilis & & & & & Glycine $\max (\mathrm{L}$.$) Merr.$ & McCall $\mathrm{AAU}, \mathrm{SSU}, \mathrm{UUU}$ & $\mathrm{N}$ & $\mathrm{N}$ \\
\hline & $\begin{array}{l}\text { Ashe] } \\
\text { Robinia pseudoacacia L }\end{array}$ & $\begin{array}{l}\operatorname{com}^{\mathrm{ENA}} \\
\mathrm{col}^{\mathrm{FNC}, \mathrm{FSB}}\end{array}$ & $\begin{array}{l}\mathrm{N} \\
\mathrm{F}\end{array}$ & F & & & (continue & he $n$ & page \\
\hline
\end{tabular}




\begin{tabular}{|c|c|c|c|c|c|c|c|c|c|}
\hline & & & Resp & nse to & & & & Respo & nse to \\
\hline & & & $\overline{\text { USDA }}$ & $\overline{\text { NGR }}$ & & & & $\overline{\text { USDA }}$ & NGR \\
\hline Familie & s, subfamilies, tribes, and species & Seed source ${ }^{a}$ & 257 & 234 & Familie & s, subfamilies, tribes, and species & Seed source ${ }^{a}$ & 257 & 234 \\
\hline & G. $\max$ & Peking ${ }^{\mathrm{PCU}}$ & $\mathrm{F}$ & $\mathrm{N}$ & & Vigna luteola (Jacq.) Benth. & $330607,406347^{\mathrm{SGU}}$ & $\mathrm{F}$ & $\mathrm{F}$ \\
\hline & G. $\max$ & Preston ${ }^{\mathrm{AAU}}$ & - & - & & Vigna minima (Roxb.) Ohwi \& Ohashi & $4985^{\mathrm{CCC}}$ & $\mathrm{F}$ & $\mathrm{F}$ \\
\hline & Glycine soja Sieb. \& Zucc. & PI81762 & $\mathrm{F}$ & $\mathrm{N}$ & & Vigna mungo (L.) Hepper & $\operatorname{com}^{\mathrm{ROG}}$ & $\mathrm{F}$ & $\mathrm{F}$ \\
\hline & Glycine tabacina (Labill.) Benth. & $\mathrm{col}^{\mathrm{PCU}}$ & - & $\mathrm{F}$ & & Vigna oblongifolia A. Rich. & $60430^{\mathrm{ASA}}$ & $\mathrm{F}$ & $\mathrm{F}$ \\
\hline & Glycine tomentella Hayata & $\mathrm{col}^{\mathrm{PUU}}$ & $\mathrm{F}$ & $\mathrm{F}$ & & Vigna parkeri Baker & Shaw ASA & $\mathrm{F}$ & $\mathrm{F}$ \\
\hline P10.36 & Teramnus labialis (L. f.) Spreng. & $60381^{\mathrm{ASA}}$ & - & $\mathrm{F}$ & & Vigna radiata $(\mathrm{L}$.$) Wilczek$ & King WSA, 305070, & $\mathrm{F}$ & $\mathrm{F}$ \\
\hline & Teramnus uncinatus (L.) Sw. & $87881^{\text {ASA }}$ & $\mathrm{N}$ & $\mathrm{F}$ & & & $197019,227754^{\mathrm{SGU}}$ & & \\
\hline P10.39 & Teyleria koordersii (Backer) Backer & $21157^{\mathrm{CCC}}$ & - & $\mathrm{N}$ & & Vigna radiata subsp. sublobata & & & \\
\hline P10.40 & Neonotonia wightii (Arn.) Lackey & Cooper's WSA & - & $\mathrm{F}$ & & (Roxb.) Verdc. & $\mathrm{col}^{\mathrm{BSU}}$ & $\mathrm{N}$ & $\mathrm{N}$ \\
\hline P10.44 & Amphicarpaea trisperma Baker & $\mathrm{col}^{\mathrm{HBC}}$ & $\mathrm{F}$ & $\mathrm{F}$ & & Vigna subterranea (L.) Verdc. & 49A, 57B $1^{\mathrm{VBI}}$ & $\mathrm{F}$ & $\mathrm{F}$ \\
\hline P10.45 & Calopogonium caeruleum (Benth.) & & & & & Vigna trilobata (L.) Verdc. & $13671^{\mathrm{ASA}}$ & $\mathrm{F}$ & $\mathrm{F}$ \\
\hline & Sauv. & $\operatorname{com}^{\mathrm{AHA}, \mathrm{IFS}}$ & - & $\mathrm{F}$ & & Vigna umbellata (Thunb.) & & & \\
\hline & Calopogonium mисипоides Desv. & $\operatorname{com}^{\text {IFS }}$ & $\mathrm{N}$ & $\mathrm{N}$ & & Ohwi \& Ohashi & $247691^{\mathrm{SGU}}$ & $\mathrm{F}$ & $\mathrm{F}$ \\
\hline P10.46 & Pachyrhizus erosus (L.) Urb. & $21039^{\mathrm{CCC}}$ & - & $\mathrm{N}$ & & Vigna unguiculata (L.) Walp. & Red Caloona ${ }^{\text {WSA }}$ & $\mathrm{F}$ & $\mathrm{F}$ \\
\hline & Pachyrhizus tuberosus (Lam.) Spreng. & $\operatorname{com}^{\mathrm{SPM}}$ & - & $\mathrm{F}$ & & Vigna vexillata (L.) A. Rich. & $406401,406390^{\mathrm{SGU}}$ & $\mathrm{F}$ & $\mathrm{F}$ \\
\hline & F Kennediinae & & & & P10.70 & Strophostyles helvola (L.) Ell. & $\mathrm{col}^{\mathrm{BMU}}$ & - & - \\
\hline P10.47 & Kennedia beckxiana F. Muell. & $\operatorname{com}^{\mathrm{ENA}}$ & $\mathrm{N}$ & $\mathrm{N}$ & P10.71 & Macroptilium atropurpureum & & & \\
\hline & Kennedia nigricans Lindley & $\operatorname{com}^{\text {ENA }}$ & $\mathrm{N}$ & $\mathrm{F}$ & & (DC.) Urb. & SiratroWSA & $\mathrm{F}$ & $\mathrm{F}$ \\
\hline & Kennedia prostrata $\mathrm{R} . \mathrm{Br}$. & $\operatorname{com}^{\mathrm{AHA}, \mathrm{AOA}}$ & $\mathrm{N}$ & $\mathrm{F}$ & & Macroptilium bracteatum & & & \\
\hline & Kennedia rubicunda (Schneev.) Vent. & $\operatorname{com}^{\mathrm{FBA}}$ & $\mathrm{N}$ & $\mathrm{F}$ & & (Nels \& Mart) Maréchal \& Baudet & RLBB62 $2^{\mathrm{WSA}}$ & $\mathrm{F}$ & $\mathrm{F}$ \\
\hline P10.48 & Hardenbergia comptoniana & & & & & Macroptilium lathyroides (L.) Urb. & $\operatorname{com}^{\mathrm{WSA}}$ & $\mathrm{F}$ & $\mathrm{F}$ \\
\hline & (Andr.) Benth. & $\operatorname{com}^{\mathrm{ADU}, \mathrm{WDA}}$ & $\mathrm{F}$ & $\mathrm{F}$ & & Macroptilium longepedunculatum & & & \\
\hline & Hardenbergia violacea (Schneer.) & & & & & (Benth.) Urb. & $575^{\mathrm{CCC}}$ & - & $\mathrm{N}$ \\
\hline & Stearn & $\operatorname{com}^{\mathrm{ISM}}$ & $\mathrm{N}$ & $\mathrm{F}$ & P10.72 & Phaseolus acutifolius A. Gray & $214333^{\mathrm{PCU}}$ & - & $\mathrm{N}$ \\
\hline & G Phaseolinae & & & & & P. acutifolius & 440813 WPU & - & $\mathrm{N}$ \\
\hline $\mathrm{P} 10.50$ & Dysolobium apioides (Gagnepain) & & & & & P. acutifolius & Tenuifolius WPU & - & $\mathrm{N}$ \\
\hline & Maréchal & $4596^{\mathrm{CCC}}$ & $\mathrm{N}$ & $\mathrm{F}$ & & Phaseolus angustifolius Roxb. & & & \\
\hline P10.51 & Psophocarpus palustris Desv. & $\operatorname{com}^{\mathrm{IFS}}$ & - & $\mathrm{F}$ & & [syn - Phaseolus anisotrichus & & & \\
\hline & Psophocarpus tetragonolobus (L.) DC. & $\operatorname{com}^{\mathrm{SPM}}$ & $\mathrm{F}$ & $\mathrm{F}$ & & Schlect.] & $312122^{\mathrm{WPU}}$ & - & $\mathrm{N}$ \\
\hline P10.56 & Otoptera burchellii DC. & $82448^{\mathrm{KRG}}$ & $\mathrm{F}$ & $\mathrm{F}$ & & Phaseolus leptostachyus Benth. & $325587^{\mathrm{WPU}} \&$ & - & $\mathrm{F}$ \\
\hline P10.61 & Lablab purpureus (L.) Sweet & Rongai ${ }^{\text {WSA }}$ & - & $\mathrm{F}$ & & & $30677^{\mathrm{CCC}}$ & & \\
\hline P10.64 & Dolichos junghuhnianus Benth. & $20030^{\mathrm{CCC}}$ & $\mathrm{F}$ & $\mathrm{F}$ & & Phaseolus coccineus $\mathrm{L}$. & $\mathrm{col}^{\mathrm{MKD}}$ & $\mathrm{N}$ & $\mathrm{N}$ \\
\hline & Dolichos trilobus $\mathrm{L}$. & $21038^{\mathrm{CCC}}$ & - & $\mathrm{F}$ & & Phaseolus coccineus L. subsp. & & & \\
\hline P10.65 & Macrotyloma axillare (E. Mey.) Verdc. & comWSA & $\mathrm{F}$ & $\mathrm{F}$ & & polyanthus (Greenman) Maréchal, & & & \\
\hline & Macrotyloma uniflorum (Lam.) Verdc. & Leichardt ${ }^{\text {ASA }}$ & - & $\mathrm{F}$ & & Mascherpa, \& Stainier & $196813^{\mathrm{WPU}}$ & $\mathrm{F}$ & $\mathrm{F}$ \\
\hline P10.66 & Vigna aconitifolia (Jacq.) Maréchal & $214323^{\mathrm{SGU}}$ & - & $\mathrm{F}$ & & Phaseolus polystachyus Britt., & & & \\
\hline & Vigna angularis (Willd.) & & & & & Stearns \& Pc & $196813^{\mathrm{WPU}}$ & - & $\mathrm{F}$ \\
\hline & Ohwi \& Ohashi & $\mathrm{col}^{\mathrm{PCU}}$ & $\mathrm{F}$ & $\mathrm{F}$ & & Phaseolus vulgaris $\mathrm{L}$. & Hilds Marona ${ }^{\text {GC }}$ & $\mathrm{N}$ & $\mathrm{N}$ \\
\hline & Vigna caracalla (L.) Verdc. & $146800^{\mathrm{SGU}}$ & - & $\mathrm{F}$ & & Phaseolus vulgaris L. var. aborigineus & & & \\
\hline & Vigna cylindrica Skeels & $\operatorname{com}^{\mathrm{ASA}}$ & $\mathrm{F}$ & $\mathrm{F}$ & & (Burk.) Baudet & $266910^{\mathrm{WPU}}$ & $\mathrm{F}$ & $\mathrm{F}$ \\
\hline & Vigna glabrescens Maréchal, & & & & & H Cajaninae & & & \\
\hline & Mascherpa \& Stainer & $207655^{\mathrm{PBU}}$ & $\mathrm{N}$ & $\mathrm{F}$ & P10.73 & Cajanus cajan (L.) Millsp. & ICP6443 ${ }^{\text {ICI }}$ & $\mathrm{F}$ & $\mathrm{F}$ \\
\hline & Vigna hosei (Craib) Backer & $4983^{\mathrm{CCC}}$ & $\mathrm{F}$ & $\mathrm{F}$ & & Cajanus scarabaeoides (L.) Thou. & $\operatorname{col}^{\mathrm{ASA}}, 87^{\mathrm{IPI}}$ & $\mathrm{F}$ & $\mathrm{F}$ \\
\hline & Vigna lanceolata Benth. & CQ592 ${ }^{\text {ASA }}$ & $\mathrm{F}$ & $\mathrm{F}$ & & & & then & pag \\
\hline
\end{tabular}




\begin{tabular}{|c|c|c|c|c|c|c|c|c|c|}
\hline & & & Resp & nse to & & & & Respo & nse to \\
\hline & & & $\overline{\text { USDA }}$ & $\overline{\text { NGR }}$ & & & & $\overline{\text { USDA }}$ & NGR \\
\hline Familie & s, subfamilies, tribes, and species & Seed source ${ }^{a}$ & 257 & 234 & Familie & s, subfamilies, tribes, and species & Seed source ${ }^{a}$ & 257 & 234 \\
\hline P10.74 & Dunbaria circinalis Backer & $17329^{\mathrm{CCC}}$ & - & $\mathrm{F}$ & & Tribe Loteae & & & \\
\hline & Dunbaria nivea Miq. & $17734^{\mathrm{CCC}}$ & - & $\mathrm{F}$ & P13.2 & Anthyllis vulneraria $\mathrm{L}$. & $55402^{\mathrm{KRG}}$ & - & $\mathrm{N}$ \\
\hline & Dunbaria villosa Mak. & $20649^{\mathrm{CCC}}$ & $\mathrm{N}$ & $\mathrm{F}$ & P13.4 & Hymenocarpos circinnatus (L.) Savi & $27229^{\mathrm{KRG}}$ & - & - \\
\hline P10.77 & Flemingia congesta Roxb. & $\operatorname{com}^{\mathrm{WSA}}$ & $\mathrm{F}$ & $\mathrm{F}$ & P13.6 & Dorycnium herbaceum Vill. & $\mathrm{col}^{\mathrm{BGD}}$ & - & - \\
\hline & Flemingia strobilifera (L.) Ait. f. & $204^{\mathrm{IPI}}$ & $\mathrm{F}$ & $\mathrm{F}$ & P13.7 & Lotus corniculatus L. & $\mathrm{col}^{\mathrm{PCU}}$ & $\mathrm{N}$ & $\mathrm{F}$ \\
\hline $\mathrm{P} 10.80$ & Rhynchosia minima (L.) DC. & $18063^{\mathrm{KRG}}$ & - & $\mathrm{F}$ & & Lotus halophilus Boiss. \& Sprun. & $26004^{\mathrm{KRG}}$ & - & $\mathrm{N}$ \\
\hline & Rhynchosia phaseoloides (Sw.) DC. & $\mathrm{col}^{\mathrm{SNU}}$ & - & - & & Lotus japonicus (Regel) K. Larsen & $\mathrm{Gifu}^{\mathrm{MLU}, \mathrm{PKU}}$ & $\mathrm{F}$ & $\mathrm{F}$ \\
\hline & Rhynchosia reticulata (Sw.) DC. & $7152^{\mathrm{CCC}}$ & - & - & & Lotus pedunculatus Cav. & Grassl. Maku ${ }^{\mathrm{APN}}$ & $\mathrm{N}$ & $\mathrm{N}$ \\
\hline & Rhynchosia rothii Benth. ex Aitch. & $30232^{\text {ASA }}$ & - & $\mathrm{F}$ & & Lotus tetragonolobus $\mathrm{L}$. & $\mathrm{col}^{\mathrm{HPP}}$ & - & - \\
\hline & Rhynchosia sublobata (Schumach.) & & & & P13.11 & Coronilla varia L. & $\mathrm{col}^{\mathrm{KLB}}$ & - & - \\
\hline & Meikle & $77003^{\mathrm{ASA}}$ & $\mathrm{F}$ & $\mathrm{F}$ & $\mathrm{P} 13.12$ & Securigera securidaca (L.) & & & \\
\hline $\mathrm{P} 10.81$ & Eriosema simplicifolium (HBK) G. & $7336^{\mathrm{CCC}}$ & - & - & & Degen \& Doerfler & $\mathrm{col}^{\mathrm{NPF}}$ & - & - \\
\hline & Don & & & & P13.13 & Hippocrepis ciliata Willd. & $26602^{\mathrm{KRG}}$ & - & - \\
\hline & Eriosema violaceum (Aubl.) G. Don & $18292^{\mathrm{CCC}}$ & - & $\mathrm{N}$ & P13.14 & Scorpiurus vermiculatus L. & $\mathrm{col}^{\mathrm{BGD}}$ & - & - \\
\hline P10.82 & Paracalyx scariosus (Roxb.) Ali & $207^{\mathrm{IPI}}$ & - & - & P13.15 & Ornithopus compressus L. & $\operatorname{com}^{\mathrm{WSA}}$ & & - \\
\hline & Tribe Desmodieae & & & & & Tribe Aeschynomeneae & & & \\
\hline & B Desmodiinae & & & & & B Aeschynomeninae & & & \\
\hline P11.6 & Dendrolobium triangulare Schindl. & $23104^{\mathrm{CCC}}$ & $\mathrm{F}$ & $\mathrm{F}$ & $\mathrm{P} 14.9$ & Aeschynomene aspera $\mathrm{L}$. & $\mathrm{col}^{\mathrm{IPI}}$ & - & $\mathrm{N}$ \\
\hline P11.7 & Phyllodium elegans (Lour.) Desv. & $23230^{\mathrm{CCC}}$ & $\mathrm{N}$ & $\mathrm{N}$ & & Aeschynomene falcata (Poir.) DC. & Barjoo $^{\mathrm{ASA}}$ & - & $\mathrm{N}$ \\
\hline & Phyllodium pulchellum (L.) Desv. & $13237^{\mathrm{CCC}}$ & - & - & & Aeschynomene indica L. & $\operatorname{col}^{\mathrm{IPI}}$ & - & $\mathrm{N}$ \\
\hline P11.8 & Dicerma biarticulatum (L.) DC. & $18401^{\mathrm{CCC}}$ & $\mathrm{N}$ & $\mathrm{N}$ & & D Poiretiinae & & & \\
\hline P11.9 & Desmodium canadense (L.) DC. & $\mathrm{col}^{\mathrm{USU}}$ & F & $\mathrm{F}$ & $\mathrm{P} 14.21$ & Zornia brasiliensis Vogel & $14287^{\mathrm{CCC}}$ & - & - \\
\hline & Desmodium dichotomum DC. & $47186^{\text {ASA }}$ & - & $\mathrm{F}$ & & Zornia diphylla Pers. & $70161^{\mathrm{CCC}}$ & - & $\mathrm{N}$ \\
\hline & Desmodium intortum Urb. & Greenleaf ${ }^{\text {YRA }}$ & - & $\mathrm{F}$ & & Zornia gemella (Willd.) Vogel & $49269^{\mathrm{KRG}}$ & - & - \\
\hline & Desmodium uncinatum (Jacq.) DC. & Silverleaf ${ }^{\text {YRA }}$ & - & $\mathrm{F}$ & & Zornia glabra Desv. & $8345^{\mathrm{CCC}}$ & - & - \\
\hline P11.10 & Codariocalyx gyroides (Roxb.) Hassk. & $13547^{\mathrm{CCC}}$ & $\mathrm{F}$ & $\mathrm{F}$ & & Zornia latifolia Sm. & $100465^{\mathrm{ASA}}$ & - & $\mathrm{N}$ \\
\hline & Codariocalyx motorius (Houtt.) Ohashi & $23414^{\text {CCC }}$ & $\mathrm{F}$ & $\mathrm{F}$ & & E Stylosanthinae & & & \\
\hline P11.11 & Pseudarthria viscida (L.) W. \& A. & $13701^{\mathrm{CCC}}$ & - & $\mathrm{N}$ & P14.23 & Pachecoa prismatica (Sessé \& Moc.) & & & \\
\hline $\mathrm{P} 11.12$ & Pycnospora lutescens (Poir.) Schindl. & $17415^{\mathrm{CCC}}$ & $\mathrm{F}$ & $\mathrm{F}$ & & Standl. \& Schub. & $18287^{\mathrm{CCC}}$ & $\mathrm{N}$ & $\mathrm{N}$ \\
\hline $\mathrm{P} 11.13$ & Tadehagi triquetrum (L.) Ohashi & $13263^{\mathrm{CCC}}$ & - & - & $\mathrm{P} 14.25$ & Stylosanthes capitata Vog. & $55843^{\mathrm{ASA}}$ & - & - \\
\hline P11.17 & Christia obcordata (Poir.) Bakh. & $20652^{\mathrm{CCC}}$ & - & - & & Stylosanthes guianensis (Aubl.) Sw. & EndeavorWSA & - & $\mathrm{N}$ \\
\hline P11.18 & Alysicarpus vaginalis (L.) DC. & $20580^{\text {CCC }}$ & - & $\mathrm{F}$ & & Stylosanthes hamata (L.) Taub. & VeranowSA & - & $\mathrm{N}$ \\
\hline & C Lespedezinae & & & & & Stylosanthes humilis Kunth & Townsville ${ }^{\mathrm{WSA}}$ & - & $\mathrm{N}$ \\
\hline $\mathrm{P} 11.24$ & Lespedeza bicolor Turcz. & $\mathrm{col}^{\mathrm{JTF}}$ & $\mathrm{F}$ & $\mathrm{F}$ & & Stylosanthes scabra Vog. & SecaASA & - & $\mathrm{N}$ \\
\hline P11.25 & Kummerowia stipulacea (Maxim.) & & & & P14.26 & Arachis hypogaea L. & $\mathrm{NC} 4 \mathrm{CRU}$ & - & $\mathrm{N}$ \\
\hline & Makino & Summit ${ }^{\mathrm{PCU}}$ & $\mathrm{F}$ & $\mathrm{F}$ & & & & & \\
\hline & Kummerowia striata (Thunb.) Schindl. & Marion $^{\mathrm{PCU}}$ & $\mathrm{F}$ & $\mathrm{F}$ & 5.1 & $\begin{array}{l}\text { Tribe Adesmieae } \\
\text { Adesmia bicolor (Poir.) DC. }\end{array}$ & $\mathrm{col}^{\mathrm{URU}}$ & - & - \\
\hline & Tribe Psoraleeae & & & & & Adesmia remyana Phil. & $\mathrm{col}^{\mathrm{ARG}}$ & - & - \\
\hline
\end{tabular}

P12.2 Bituminaria bituminosa (L.) C.H. Stirton

P12.9 Psoralea plumosa F. Muell.

Psoralea pustulata F. Muell. 


\begin{tabular}{|c|c|c|c|c|c|c|c|c|c|}
\hline & & & Resp & onse to & & & & Resp & onse to \\
\hline & & & $\overline{\text { USDA }}$ & NGR & & & & $\overline{\text { USDA }}$ & NGR \\
\hline Familie & es, subfamilies, tribes, and species & Seed source ${ }^{a}$ & 257 & 234 & Famili & , subfamilies, tribes, and species & Seed source ${ }^{a}$ & 257 & 234 \\
\hline & Tribe Galegeae & & & & & Vicia narbonensis L. & $13167^{\mathrm{ASC}}$ & - & - \\
\hline & A Coluteinae & & & & & Vicia sativa L. subsp. nigra (L.) Ehrh. & $\operatorname{com}^{\mathrm{ILF}}$ & - & - \\
\hline P16.1 & Clianthus formosus (G. Don) & & & & & Vicia pannonica Crantz & Pannonica ${ }^{\mathrm{ASC}}$ & - & - \\
\hline & Ford \& Vick & $\operatorname{com}^{\mathrm{AHA}}$ & - & - & & Vicia villosa Roth & & & \\
\hline P16.2 & Swainsona cyclocarpa F. Muell. & Paradoxa ${ }^{\mathrm{KPA}}$ & - & - & & subsp. pseudocracca (Bertol.) Ball & Glabrecens ${ }^{\text {ASC }}$ & - & - \\
\hline & Swainsona formosa (G. Don) & & & & & Vicia vulgaris Moench & $\operatorname{com}^{\mathrm{ASC}}$ & - & - \\
\hline & J. Thompson & $\operatorname{com}^{\mathrm{ADU}, \mathrm{NAA}}$ & - & - & $\mathrm{P} 19.2$ & Lathyrus japonicus Willd. & $\mathrm{col}^{\mathrm{JMC}}$ & - & - \\
\hline & Swainsona forrestii F. Muell. ex A. & $\operatorname{col}^{\mathrm{KPA}}$ & - & $\mathrm{F}$ & & Lathyrus magellanicus Lam. & $\operatorname{col}^{\mathrm{PPA}}$ & - & - \\
\hline & 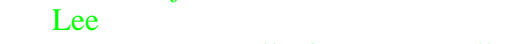 & & & & & Lathyrus odoratus L. & $\operatorname{com}^{\mathrm{TJU}}$ & - & - \\
\hline & Swainsona maccullochiana F. Muell. & $\operatorname{com}^{\mathrm{HCA}}$ & - & - & P19.3 & Lens culinaris Medik. & $\mathrm{col}^{\mathrm{PCU}}$ & - & - \\
\hline & $\begin{array}{l}\text { Swainsona procumbens (F. Muell.) } \\
\text { F. Muell. }\end{array}$ & & - & - & P19.4 & Pisum sativum $\mathrm{L}$. & com ASC & & \\
\hline P16.3 & Sutherlandia frutescens (L.) R. Br. & $\mathrm{col}^{\mathrm{BBD}}$ & - & - & & $\begin{array}{l}\text { subsp. elattus (B1eb.) Asch. \& Gr. } \\
\text { Pisum sativum L. }\end{array}$ & $\begin{array}{l}\text { Rom }^{\text {Rondo }} \\
\text { RRN }^{2}\end{array}$ & $\begin{array}{l}- \\
-\end{array}$ & $\begin{array}{l}- \\
-\end{array}$ \\
\hline $\mathrm{P} 16.5$ & Colutea arborescens $\mathrm{L}$. & $\mathrm{col}^{\mathrm{BGD}, \mathrm{HPP}}$ & $\mathrm{F}$ & $\mathrm{F}$ & & & & & \\
\hline & B Astragalinae & & & & & Tribe Cicereae & & & \\
\hline P16.10 & $\begin{array}{l}\text { Halimodendron halodendron } \\
\text { (Pallas) Voss }\end{array}$ & $\mathrm{col}^{\mathrm{HBC}}$ & $\mathrm{N}$ & $\mathrm{N}$ & P20.1 & Cicer arietinum $\mathrm{L}$. & Annigeri ${ }^{\mathrm{IPI}}$ & - & - \\
\hline P16.11 & Caragana arborescens Lam. & $\mathrm{com}^{\mathrm{FBA}, \mathrm{JMF}}$ & - & - & & Tribe Trifolieae & & & \\
\hline P16.15 & Astragalus alpinus L. & $\mathrm{col}^{\mathrm{NPF}}$ & - & - & $\mathrm{P} 21.1$ & Ononis natrix L. & $\mathrm{col}^{\mathrm{CGC}}$ & - & - \\
\hline & Astragalus canadense $\mathrm{L}$. & & & & $\mathrm{P} 21.3$ & Melilotus officinalis Willd. & $31169^{\mathrm{KRG}}$ & - & - \\
\hline & [syn - Astragalus cicer L.] & $\operatorname{com}^{\mathrm{PWU}}, \mathrm{col}^{\mathrm{CGC}}$ & - & - & P21.4 & Trigonella foenum-graecum $\mathrm{L}$. & $\operatorname{col}^{\mathrm{CGC}}$ & - & - \\
\hline & Astragalus cruikschankii Griseb. & $\mathrm{col}^{\mathrm{PPA}}$ & - & - & & Trigonella gladiata $\mathrm{M}$. Bieb. & $55240^{\mathrm{KRG}}$ & - & - \\
\hline P16.17 & Oxytropis campestris (L.) DC. & $\mathrm{col}^{\mathrm{BJF}, \mathrm{HPP}}$ & - & - & $\mathrm{P} 21.5$ & Medicago arabica (L.) Huds. & $\mathrm{col}^{\mathrm{WPU}}$ & - & - \\
\hline & Oxytropis halleri Bunge ex Koch & $36108^{\mathrm{KRG}}$ & $\mathrm{F}$ & $\mathrm{F}$ & & Medicago arborea $\mathrm{L}$. & $\mathrm{col}^{\mathrm{WPU}}$ & - & - \\
\hline & Oxytropis uralensis DC. & & & & & Medicago blancheana Boiss. & $\mathrm{col}^{\mathrm{WPU}}$ & - & - \\
\hline & [syn - Oxytropis arctica $\mathrm{R}$. Br.] & $\mathrm{col}^{\mathrm{JMC}}$ & - & - & & Medicago brachycarpa M. Bieb. & $\mathrm{col}^{\mathrm{WPU}}$ & - & - \\
\hline P16.19 & Gueldenstaedtia stenophylla Bunge & $\mathrm{col}^{\mathrm{HBC}}$ & - & $\mathrm{N}$ & & Medicago cancellata M. Bieb. & $\mathrm{col}^{\mathrm{WPU}}$ & $N^{\star}$ & $N^{\downarrow}$ \\
\hline & C Galeginae & & & & & Medicago ciliaris (L.) Krocker & $\mathrm{col}^{\mathrm{WPU}}$ & - & - \\
\hline P16.20 & Galega officinalis L. & $36670^{\mathrm{KRG}}$ & - & - & & Medicago constricta Durien & $\mathrm{col}^{\mathrm{WPU}}$ & - & - \\
\hline & E Glycyrrhizinae & & & & & Medicago coronata (L.) Bart. & $\mathrm{col}^{\mathrm{WPU}}$ & - & - \\
\hline P16.22 & Glycyrrhiza acanthocarpa (Lindl.) & & & & & Medicago disciformis DC. & $\mathrm{col}^{\mathrm{WPU}}$ & - & - \\
\hline & J.M. Black & $\mathrm{col}^{\mathrm{KPA}}$ & - & - & & Medicago doliata Carmign. & Muricata ${ }^{W P U}$ & - & - \\
\hline & Glycyrrhiza glabra L. & $\mathrm{col}^{\mathrm{KLB}}$ & - & $\mathrm{F}$ & & Medicago granadensis Willd. & $\mathrm{col}^{\mathrm{WPU}}$ & - & - \\
\hline & & & & & & Medicago heyniana Greuter & $\mathrm{col}^{\mathrm{WPU}}$ & - & - \\
\hline & Tribe Hedysareae & & & & & Medicago italica (Miller) Fiori & $\mathrm{col}^{\mathrm{WPU}}$ & - & - \\
\hline $\mathrm{P} 18.2$ & Hedysarum alpinum L. & $\mathrm{col}^{\mathrm{BBD}}$ & $\mathrm{N}$ & $\mathrm{N}$ & & Medicago laciniata (L.) Miller & $\mathrm{col}^{\mathrm{WPU}}$ & - & - \\
\hline & Hedysarum coronarium $\mathrm{L}$. & $\operatorname{com}^{\mathrm{TJU}}$ & - & - & & Medicago lesinsii E. Small & $\mathrm{col}^{\mathrm{WPU}}$ & - & - \\
\hline P18.6 & Onobrychis caput-galli (L.) Lam. & $\mathrm{col}^{\mathrm{BBD}}$ & - & - & & Medicago littoralis Rohde ex Lois. & $\mathrm{col}^{\mathrm{WPU}}$ & - & - \\
\hline & Onobrychis viciifolia Scop. & $\mathrm{col}^{\mathrm{CBC}}$ & - & - & & Medicago medicaginoides (Retz.) & & & \\
\hline & & & & & & E. Smith & $\mathrm{col}^{\mathrm{WPU}}$ & - & - \\
\hline & Tribe Vicieae & & & & & Medicago minima (L.) Bart. & $\mathrm{col}^{\mathrm{WPU}}$ & - & - \\
\hline P19.1 & Vicia benghalensis L. & $\operatorname{com}^{\mathrm{BWU}}$ & - & - & & Medicago monantha (C. A. Meyer) & & & \\
\hline & Vicia cracca L. & $\mathrm{col}^{\mathrm{BJF}}$ & - & - & & Trautv. & $\mathrm{col}^{\mathrm{WPU}}$ & - & - \\
\hline & Vicia dasycarpa Ten. & $\operatorname{com}^{\mathrm{FBA}}$ & - & - & & & - & he 1 & page) \\
\hline & Vicia faba $\mathrm{L}$. & $\mathrm{col}^{\mathrm{CGC}}$ & - & - & & & & net & \\
\hline
\end{tabular}




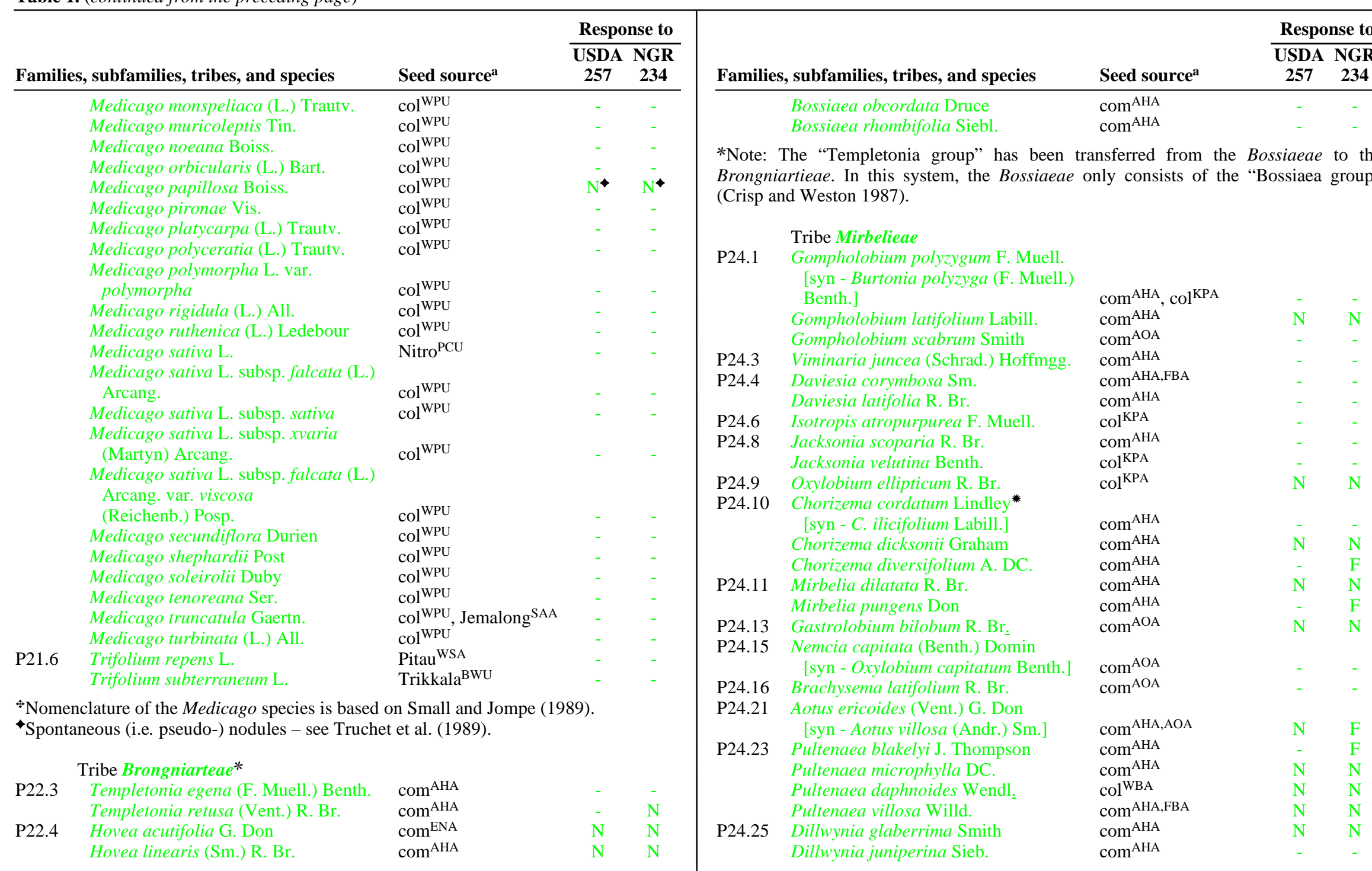

*Note: Chorizema cordatum was originally acquired as Chorizema ilicifolium, from

Tribe Bossiaeae*

P23.5 Goodia lotifolia Salisb.

P23.6 Bossiaea foliosa A. Cunn.

Bossiaea heterophylla Vent. $\quad \operatorname{com}^{\mathrm{AHA}}$

which it segregates (Taylor and Crisp 1992). 


\begin{tabular}{|c|c|c|c|c|c|c|c|c|c|}
\hline & & & Respo & nse to & & & & Respo & nse to \\
\hline & & & $\overline{\text { USDA }}$ & $\overline{\text { NGR }}$ & & & & $\overline{\text { USDA }}$ & NGR \\
\hline Famili & , subfamilies, tribes, and species & Seed source ${ }^{a}$ & 257 & 234 & Familie & , subfamilies, tribes, and species & Seed source ${ }^{a}$ & 257 & 234 \\
\hline D25 2 & Tribe Podalyrieae & & & & P30.9 & Laburnum anagyroides Medik. & CGC JTF & & $\mathrm{N}$ \\
\hline P25.3 & $\begin{array}{l}\text { Virgilia capensis (P. Bergius) } \\
\text { T.M. Salter subsp. oroboides }\end{array}$ & & & & & $\begin{array}{l}\text { [syn - Laburnum } \mathrm{x} \text { watereri] } \\
\text { Laburnum vossii Hort. }\end{array}$ & $\mathrm{com}^{\mathrm{FBA}}$ & - & $\begin{array}{l}\mathrm{N} \\
\mathrm{N}\end{array}$ \\
\hline & [syn - Virgilia capensis (L.) Lam] & $\operatorname{com}^{\mathrm{AHA}}$ & - & $\mathrm{F}$ & P30.10 & Hesperolaburnum platycarpum & & & \\
\hline & Virgilia divaricata Adamson & $\mathrm{col}^{\mathrm{ROG}}$ & - & $\mathrm{N}$ & & (Maire) Maire & $84176^{\mathrm{KRG}}$ & - & $\mathrm{F}$ \\
\hline & & & & & P30.15 & Cytisus hirsutus L. & $\operatorname{com}^{\text {FBA }}$ & - & $\mathrm{N}$ \\
\hline & Tribe Crotalarieae & & & & & Cytisus villosus Pourret & $\mathrm{com}^{\mathrm{FBA}}$ & $\mathrm{N}$ & $\mathrm{N}$ \\
\hline P27.7 & Crotalaria juncea L. & $\operatorname{com}^{\mathrm{IFS}}$ & $\mathrm{N}$ & $\mathrm{N}$ & P30.15 & Chamaecytisus proliferus (L.f.) Link & & & \\
\hline & Crotalaria sericea Retz. & $\mathrm{col}^{\mathrm{PCU}}$ & $\mathrm{N}$ & $\mathrm{F}$ & & subsp. palmensis (Christ) Kunkel & $66477^{\mathrm{KRG}}$ & - & $\mathrm{N}$ \\
\hline P27.9 & Lotononis bainesii Bak. & comWSA & - & - & P30.16 & Lembotropis nigricans (L.) Griseb. & $88510^{\mathrm{KRG}}$ & $\mathrm{N}$ & $\mathrm{N}$ \\
\hline & & & & & P30.17 & Calicotome villosa (Poir.) Link & $31963^{\mathrm{KRG}}$ & & $\mathrm{N}$ \\
\hline & Tribe Thermopsideae & & & & P30.19 & Spartium junceum L. & $\mathrm{col}^{\mathrm{JTF}}$ & $\mathrm{N}$ & $\mathrm{N}$ \\
\hline P29.2 & Piptanthus concolor Harrow & $\mathrm{col}^{\mathrm{HBC}}$ & - & $\mathrm{F}$ & $\mathrm{P} 30.21$ & Retama monosperma (L.) Boiss. & $84349^{\mathrm{KRG}}$ & - & $\mathrm{F}$ \\
\hline P29.3 & Anagyris foetida $\mathrm{L}$. & $\mathrm{col}^{\mathrm{BBD}}$ & - & $\mathrm{F}$ & & Retama raetam (Forsskal) Webb & $\operatorname{com}^{\text {FBA }}$ & - & $\mathrm{F}$ \\
\hline P29.4 & Thermopsis caroliniana & & & & P30.22 & Genista pilosa $\mathrm{L}$. & $\mathrm{col}^{\mathrm{CGC}}$ & - & - \\
\hline & M. A. Curt. & $\mathrm{col}^{\mathrm{SNU}}$ & - & - & & Genista tinctoria $\mathrm{L}$. & $\mathrm{col}^{\mathrm{CGC}}$ & - & - \\
\hline $\mathrm{P} 29.5$ & Baptisia australis (L.) R. Br. & $\mathrm{col}^{\mathrm{BNU}, \mathrm{UOG}}$ & - & - & P30.23 & Echinospartum horridum (Vahl) Rothm. & $\mathrm{col}^{\mathrm{CGC}}$ & - & - \\
\hline & & & & & P30.25 & Ulex europaeus $\mathrm{L}$. & $\mathrm{col}^{\mathrm{BBD}, \mathrm{HPP}}$ & - & $\mathrm{N}$ \\
\hline & Tribe Genisteae & & & & Note - & Lupinus nodules are unusual, and for this & reason have no & lassified & here. \\
\hline P30.8 & $\begin{array}{l}\text { Lupinus albus } \mathrm{L} . \\
\text { Lupinus angustifolius } \mathrm{L} .\end{array}$ & $\begin{array}{l}\text { Kijevsky mutant }{ }^{\mathrm{ASC}} \\
\text { col }^{\mathrm{APN}}\end{array}$ & - & - & & & & & \\
\hline & Lupinus luteus L. & Jantar ASC & - & - & & & & & \\
\hline & Lupinus nanus Dougl. & $\mathrm{col}^{\mathrm{APN}}$ & - & - & Paraspo & nia andersonii Planch. & tiss cul & $\mathrm{N}$ & $\mathrm{N}$ \\
\hline & Lupinus pilosus L. & $79170^{\text {кко }}$ & - & $\mathrm{N}$ & $\succ_{\text {Note }}$ & Experiments were performed on plants & ised in tissue & & \\
\hline
\end{tabular}
awhere: col = collected seeds; com = commercial variety; names are those of varieties/cultivars; figures represent the supplier's accession number; and superscripts are abbreviations
for the suppliers whose addresses are listed below.

${ }^{a}$ Seed sources are as follows: AAU = Agronomy Department, Iowa State University, Ames 50011-1010, U.S.A. (W. Fehr, J. Orf); ACA = Australian Tree Seed Centre, CSIRO Division of Forestry, PO Box 4008, Queen Victoria Terrace, Canberra 2600, AUS (C. Gardiner); ADU = Australian Products Inc., 7502 Greenvile - Suite 500, Dallas, TX 75231, U.S.A.; AHA = Australian Seed Co., PO Box 67, Hazelbrook, NSW 2779, AUS (A. J. Bourne); AOA = Australian Revegetation Corp., 42 Sarich Court, Osborne Park, 6017 AUS (W. Haines); APN = Applied Biochemistry Division, Department of Scientific and Industrial Research, Palmerston North, NZ; ASA = Australian Tropical Forages Genetic Resources Centre, CSIRO Division of Tropical Crops and Pastures, Cunningham Laboratory, 306 Carmody Rd., St. Lucia, 4067 AUS (B. Pengelly); ASC = Agritec Research, Breeding \& Services Ltd., Zemedelska 16, 78701 Sumperk-Temenice, CR (M. Hybl); AWU = W. Atlee Burpee Co., Warminster, PA 18974, U.S.A.; BAD = Botanisk Institut, Aarhus Universitet, Nordlandsvej 68, 8240, DK (K. Tybirk); BBD = Botanischer Garten und Botanisches Museum, Berlin-Dahlem, Königin-Luise-Straße 6-8, 14195 Berlin, D; BGD = Botanischer Garten, der Universität, Untere Karspüle 1, 37073 Göttingen, D; BJF = Botanical Gardens, University of Joensuu, PO Box 111, 80101 Joensuu 10, FI; BMU = Biochemistry Department, Kansas State University, Manhattan 65506, U.S.A. (L. Davis); BNG = Biology Department, The University, Newcastle Upon Tyne, NE1 7RU, GB; BSS = Hortus Botanicus Bergianus, University of Stockholm, PO Box 50017, 10405 Stockholm, S; BSU = Department of Plant Pathology, University of Minnesota, 495 Borlaug Hall, 1991 Upper Buford Circle, St. Paul 55108, U.S.A. (N. D. Young); BTC = Banco Latinamericano de Semilas Forestales, CATIE, Turrialba, CR (E. Ledema); BWU = Bountiful Gardens, Willits, CA 95490, U.S.A.; CBC = Ceskoslovenska Akademie Ved, Ustav experimentalni botaniky, 37005 Ceske Budejovice, CZ; CCC = Centro Internacional de Agricultura Tropical (CIAT), Apdo. aéro 6713, Cali, CO (B.L. Maass, A. Ortiz, and R. Schultze-Kraft); CGC = Conservatoire et Jardin botaniques de la Ville de Genève, PO Box 60, 1292 Chambésy/Genève, CH (G. Lachard); CNF = Conservatoire et Jardins Botaniques de Nancy, 100 rue du Jardin Botanique, 54600 Villiers les Nancy, F; CRN = Cebe \& Co., 
(continued from the preceding page)

Blaak 31, 3011 GA, Rotterdam, NL; CRU = Crop Science Department, North Carolina State University, Raleigh 27650, U.S.A. (J. C. Wynne); CSI = Conservatoire et Jardin Botanique de Mascarin, Domaine des Colimaçons, 97436 Saint-Leu, Ile de la Réunion, F; DHD = Danida Forest Seed Centre, Krogerupvej 3 A, 3050 Humlebk, DK (K. Poulsen); DNF = Département forestier CIRAD-Forêt, 45 bis, av. de la Belle Gabrielle, 94736 Nogent s/Marne, F; DPA = Darwin Botanic Gardens, PO Box 496, Palmerston 0831, AUS; ENA = Ellison Horticultural, PO Box 365, Nowra 2541, AUS; FBA = M.L. Farrar Pty. Ltd. (now AustraHort Pty. Ltd.), PO Box 1046 Bomaderry 2541, AUS; FDA = Fruit Spirit Botanical Garden, Dorroughby, 2480 AUS (P. Recher); FNC = Forestry Department, Ministry of Agriculture and Natural Resources, Nicosia, CY (S. P. Soteriou); FSB = Forest Research Insitute, 132 Blv. Kliment Ohridski, 1756 Sofia, BG (V. Bouzov and A. Delkov); FSR = FAO-UN, Avda. Santa Maria 6700, Casilla 10095, Santiage, RCH; FZM = Forest Research Institute of Malawi, PO Box 270, Zomba, MW (J. P. Gowela); GKM = Institut Penyelidikan Getah Malaysia, Biotechnology Division, 260 Jalan Ampang, Petisurat 10150, 50908, Kuala Lumpur, Malaysia; HAN = Hortus Botanicus Plantage, Plantage Middenlaan 2, 1018 DD Amsterdam, NL; HBC = Hortus Botanicus Pekinensis, Instituti Botanici Academiae Sinicae, Xiangshan, Beijing 100093, China (Z.-M. Zhang); HBU = Horticulture Department, Louisiana State University, Baton Rouge 70803, U.S.A. (W. J. Blackmon); HCA = Harper Seed Co., PO Box 315, Cannington 6107, AUS; HPP = Hortus Botanicus, Universitatis Posnaniensis, Dqbrowskiego 165, 60594 Posnan, PL; IAE = International Livestock Centre for Africa, PO Box 5689, Addis Ababa, ETH (J. Hanson); IFS = Inland and Foreign Trading Co., Block 79A Indus Road, Singapore 0316, SGP; ILF = INRA-SAPF, 86600 Lusignan, F; IPI = ICRISAT, Patancheru, Andhra Pradesh 502 324, IND (A. M. Ghanekar); JMC = Jardin Botanique de Montréal, rue Sherbrooke, Montréal, Quebec H1X 2B2, CDN; JMF = Jardins Botaniques de Marseille, 48 Av. Clot-Bey, 13008 Marseille, F; JRF = Jardin Botanique, 7 rue de Trianon, 76100 Rouen, F; JTF = Jardin Botanique, Laboratoire de Biologie Végétale, 37042 Tours Cedex, F; KPA = Kings Park and Botanical Garden, West Perth 6005, AUS KLB = Kruidtuin van de Stad Leuven, Hortus Botanicus Lovaniensis, 30 Kapucijnenvoer, 3000 Leuven, B; KRG = Royal Botanical Gardens Seed Bank, Wakehurst Place, Ardingly, Haywards Heath, West Sussex RH17 6TN, GB (J. Bone); MKD = Max-Planck-Institut für Züchtungsforschung, Carl von Linné Weg, 50829 Köln, D (E. Czerny); MLU = MSU-DOE Plant Research Laboratory, Michigan State University, East Lansing 48825, U.S.A. (F. J. de Bruijn); NAA = Natseeds, 5 Reynolds Rd., Applecross, 6153, AUS; NHU = NifTAL Project, 1000 Holomua Av., Paia, Maui, HI 96779-9774, U.S.A.; NHZ = National Herbarium \& Botanic Garden, PO Box 8100, Causeway, Harare, Zimbabwe (T. Muller); NMA = Native Plant Section, Woods \& Forests, Bremer Rd., PO Box Murray Bridge, 5253 AUS (J. Fairlamb); NMU = Northrup King Co., Horticulture Div., PO Box 959 Minneapolis, MN 55440, U.S.A.; NPF = Muséum d'Histoire Naturelle-Cultures, 43 Rue Buffon, 75005, Paris, F; NWU = Nitrogen Fixing Tree Association (NFTA), PO Box 680, Waimanolo, HI 96795, U.S.A.; OCC = OSEVA, Zemedelska16, 78701 Sumperk-Temenice, CZ (M. Hybl); ODS = OSTROM, Laboratoire de Biologie des Sols, PB 1386 Dakar, SN; PBE = Parcs I Jardins, Escola de Jardineria, Av. Marquès de Comillas, s/n. Parc de Monthuïc, 08038 Barcelona, E; PBU = Plant Introduction Office, USDA-ARS-PSI-NGRL-PIO, Barc-West, Beltsville, MD 20705, U.S.A.; PCU = Department of Plant Pathology, University of Missouri, 108 Waters Hall, Columbia 65211, U.S.A. (S. G. Pueppke); PKU = Plant Molecular Genetics, 269 Ellington Plant Sci. Bldg., University of Tennessee, Knoxville 37901-1071, U.S.A. (P. M. Gresshoff); PPA = Universidad Nacional de la Patagonia, cc No. 786, 900 Comodoro Rivadavia, Patagonia, Argentina (M. Stronati); PPM = Pusat Penyelidikan Sains Kajihayat, Universiti Sains Malaysia, Pulau Pinang, MAL (Wong Chee-Hoong, Zairi Jaal); PTJ = Faculty of Pharmaceutical Sciences, University of Tokyo, Bunkyo-ku, Tokyo 113, JP (U. Sankawa); PUU = Perennial Glycine Germplasm Collection, Department of Crop Science, University of Illinois, AE-110 Turner Hall, 1102 S. Goodwin Ave., Urbana 61801, U.S.A. (T. Hymowitz); PWU = Prairie Moon Nursery, Route 3, Box 163, Winona, MN 55987, U.S.A.; ROG = Rawlings Seeds, 5 Worlds End Lane, Green Street Green, Orpington, Ken BR6 6AA, GB (W. Rawlings); SAA = South Australian Seed Growers Cooperative, 135 Sth Terrace, Adelaide 5000, AUS; SCP = Sistemas Agroecologicos de Manejo Comunal en la Costa Norte del Peru, Los Alhelies 164, Santa Victoria, Chiclayo, PE; SGU = Southern Regional Plant Introduction Station (USDA), Athens, GA 30602, U.S.A.; SNU = Botanic Garden of Smith College, Northampton, MA 01063, U.S.A.; SPM = Soon Huat Seeds Co., 245 Beach St., 10300 Penang, MAL; SPZ = Seed Section, Department of Water Affairs and Forestry, Forestry Branch, PO Box 727, 0001 Pretoria, ZA.; SSU = Department of Soil, Water, and Climate, University of Minnesota, Borlaug Hall, 1991 Upper Buford Circle, St. Paul 55108, U.S.A. (P. H. Graham and M. J. Sadowsky); TJU = Thompson \& Morgan Seed Co., Jackson, NJ 08527, U.S.A.; TSU = Tropical Seeds, PO Box 11122, Honolulu, HI 96828, U.S.A.; UBR = UPT Balai Pengembangan Kebun Raya-LIPI, Jl. Ir. H. Juanda 13, PO Box 309, Bogor, 16003, RI (Ir. Suhirman); UGC = Université de Genève, 1 ch. de l'Impératrice, 1292 Chambésy/Genève, CH (W. J. Broughton); ULA = Institute of Plant Physiology, cc 327, Universidad Nacional de La Plata, La Plata, 1900 Argentina (A. Arambarri); UOG = University of Oxford Botanic Garden, Rose Lane, Oxford OX1 4AX, GB; USU =_Universidad de la Republica, SayagoMontevido, Uruguay; UUU = USDA Soybean Germplasm Collection, USDA-Agricultural Research Service, University of Illinois, 232 EASB, 1101 West Peabody Drive, Urbana 61801, U.S.A. (R. Nelson); VBI = Department of Legume Inoculation, The Volcani Center, PO Box 6, Bet Dagan 50-250, Israel; WBA = Wildlife Reserve, LaTrobe University, Bundoora, Victoria 3083, AUS (B. C. Smith); WDA = West Australian Wildflower Seed Co., Darlington 6070, AUS (D. M. Wilson); WPU = Western Regional Plant Introduction Station, 59 Johnson Hall, Washington State University, Pullman 99164, U.S.A. (D. Stout); WSA = Wrightson Seeds., PO Box 357, Seven Hills 2147, AUS (S. Mitchell and H. Osborn); YRA = Arthur Yates Co., 25 Stanley St., Rockhampton 4700, AUS 
sole exceptions to these rules. On these plants, the nodules produced by USDA257 were Fix ${ }^{+}$, while those produced by NGR234 were Nod $^{+} \mathrm{Fix}^{-}$. Perhaps this is understandable with the Glycine spp., because their center of origin is in northeast China where $R$. fredii was first isolated (Hymowitz and Newell 1980; Keyser et al. 1982). It is less obvious with A. americana, however, as this species is indigenous to North America (Allen and Allen 1981).

\section{NGR234, USDA257, and the "cowpea miscellany."}

As mentioned above, both NGR234 and USDA257 are highly compatible with genera of the Phaseoleae, particularly the subtribe Phaseolinae, which includes the genus Vigna. Coincidentally, this is a defining characteristic of the "cowpea miscellany" (see above). To examine further these affinities, we compared the NGR234 and USDA257 host ranges with those of classic "cowpea" strains. Three different Bradyrhizobium strains were used, including CB756, which is widely used as an inoculant and is known to have a broad host range (Diatloff and Date 1978). Also included were USDA76 and USDA110, the latter of which is by far the best-studied Bradyrhizobium strain (see Göttfert 1993). We also included $R$. loti NZP4010 along with the fast-growing isolates because it has a moderately broad host range (Lewin et al. 1987b) even though its symbiotic genes are borne on the chromosome (Pankhurst et al. 1983b; Chua et al. 1985; Scott et al. 1996).

The nodulation capacities of all six bacteria were tested on three different Vigna spp. Only NGR234 and USDA257 were able to nodulate all plants (Table 3), and even then both were Fix $^{-}$on V. radiata subsp. sublobata. In comparison, NZP4010 only nodulated $30 \%$ of the test plants, while the nodulation capacities of the Bradyrhizobium strains were in the range of 80 to $90 \%$. Since NGR234 and USDA257 nodulate more Vigna species and cultivars than conventional "cowpea" bradyrhizobia, they obviously possess the host-range requirements of this group and could, for this reason, be considered part of it.

\section{Nodule morphology.}

Although it is clear that the legume controls nodule structure, much useful symbiotic information can be obtained by comparing the effects of different rhizobia on nodulation of the same or different plants. As examples, Dilworth (1969) examined the effect of changing the plant species (Lupinus luteus and Ornithopus sativus) while maintaining a constant bacterial component (Bradyrhizobium lupini) on leghemoglobin synthesis. By inoculating $V$. unguiculata with either a Bradyrhizobium sp. or with NGR234, Broughton and Dilworth (1971) were able to confirm that the plant encodes the globin portion of the molecule. Extension of host-range studies, in which a nonreactive strain is complemented for nodulation by genetic loci derived from homologous rhizobia (Broughton et al. 1984, 1986), allowed identification of species-specific nodulation genes (Lewin et al. 1987a; 1990; Krishnan et al. 1992; Fellay et al. 1995a; Hanin et al. 1997; Jabbouri et al. 1998). Correlations have been made between the activity of these genes, their effect on Nod-factor structure, and their ability to nodulate specific plants (Jabbouri et al. 1995; Hanin et al. 1997; Quesada-Vincens et al. 1997; Jabbouri et al. 1998; Berck et al. 1999).

Some combinations of legume species that are $\mathrm{Fix}^{+}$with NGR234 and Nod $^{-}$with USDA257 have proven useful in elu- cidating Nod-factor structures and the plant requirement for nodulation. Leucaena leucocephala is an example. L. leucocephala not only possess indeterminate nodules, but it is one of only two Mimosoid plants that are $\mathrm{Nod}^{-}$with USDA257 and Fix ${ }^{+}$with NGR234 (Table 1). For these reasons, it has been widely used in extension of host-range studies. It is now known that N-methylated Nod factors are necessary for nodulation of L. leucocephala and L. diversifolia (Fig. 2). Similarly, Calopogonium caeruleum and Pachyrhizus tuberosus, which have determinate nodules, are $\mathrm{Nod}^{-}$with USDA257 and Fix ${ }^{+}$with NGR234 and have been helpful in the elucidation of Nod-factor structures (see below).

\section{Model legumes.}

Spurred on by the success attained when numerous research groups concentrated their efforts on Arabidopsis thaliana (L.) Heynh., molecular biologists have sought a comparable legume for genetic manipulation. Ideally, such a legume should (i) be diploid, (ii) have few, morphologically distinct chromosomes, (iii) possess a small genome, (iv) be autogamous, (v) possess simple and well-developed genetics, (vi) have a rapid generation time, (vii) be transformable, (viii) grow readily under laboratory conditions, and (ix) have the capacity to nodulate with a wide range of rhizobia. Obviously, model plants would be of more interest if they were also economically important.

Surprisingly, very few of the widely used or studied legumes meet these criteria. Arachis hypogaea, G. max, and Medicago sativa are tetraploids, while the genome of $P$. sativum is much larger than that of humans. Of the widely traded legumes, only Phaseolus and Vigna spp. possess relatively small genomes (500 to $600 \mathrm{Mbp} / 1 \mathrm{C}$ ) and are true diploids (Arumuganathan and Earle 1991). Both are difficult to transform and regenerate, however. Partly for this reason, two other legumes have been adopted as models: Lotus japonicus (Handberg and Stougard 1992; Jiang and Gresshoff 1997; Stiller et al. 1997; Szczyglowski et al. 1998) and Medicago truncatula (Barker et al. 1990). Rhizobium loti is the symbiont of L. japonicus, while M. truncatula associates with $R$. meliloti. As the molecular genetics of $R$. loti are not very well developed, however, the finding that both NGR234 and USDA257 effectively nodulate $L$. japonicus removes this obstacle to the establishment of L. japonicus as a model system (Table 1). Many nodulation and other mutants of NGR234 exist, and the complete nucleotide sequence of the symbiotic plasmid (Freiberg et al. 1997) should make up for the lack of molecular information on $R$. loti strains. Of course, the ability of USDA257 and NGR234 to nodulate G. $\max$ and $V$. unguiculata, respectively, has also allowed intensive study of these hosts (e.g., Trese and Pueppke 1991; Krause et al. 1994; Arsenijević-Maksimović et al. 1997; Gehring et al. 1997).

\section{Correlations between Nod factors and nodulation.}

Although the concept of host specificity is nebulous, hostspecificity of nodulation (hsn) loci, which are unique to specific rhizobia, direct the adjunction of unique groups to the core lipo-oligosaccharide Nod factors, and should thus permit nodulation of certain legumes. For example, stem nodules on Sesbania rostrata are only induced by Azorhizobium caulinodans, a bacterium that produces arabinosylated Nod factors (Mergaert et al. 1993). Host specificity, while not so stringent in other legume-Rhizobium combinations, can also restrict the 
pool of possible microsymbionts to a few rhizobia. $R$. leguminosarum bv. viceae, which nodulates $P$. sativum and some $V i$ cia spp., produces Nod factors in which the acyl-chain is highly unsaturated (Spaink et al. 1991). Perhaps the clearest examples concern $M$. sativa and $R$. meliloti. Wild-type $R$. meliloti Nod factors are sulfated $\{\operatorname{NodRm}(\mathrm{IV}, \mathrm{V})[\mathrm{Ac}, \mathrm{S}]\}$, while those of $\mathrm{NodH}^{-}$mutants, which are unable to nodulate M. sativa, are not $\{\operatorname{NodRm}(\mathrm{IV}, \mathrm{V})[\mathrm{Ac}]\}$. Yet, $\mathrm{NodH}^{-}$mutants nodulate Vicia sativa subsp. nigra, which wild-type $R$. meliloti is unable to do (Roche et al. 1991a). Although these results are clouded by the varying phenotype of some $\mathrm{NodH}^{-}$mutants (Ogawa et al. 1991), they suggest that $M$. sativa requires sulfated Nod factors for nodulation while $V$. sativa does not tolerate them.

NGR234 and USDA257 represent the other extreme. As shown here, NGR234 is able to nodulate more than $50 \%$ of all legumes on which it was tested. Obviously, nod genes (and to a large extent, Nod factors) are involved in these varying patterns of nodulation. At first sight, some genes seem to function like $\operatorname{nodH}$. noeE, which is involved in sulfation of the 2$O$-methylfucose residue of NodNGR factors, is essential for nodulation of Calopogonium caeruleum (Hanin et al. 1997). Gaps within the nod box promoter region and the $5^{\prime}$ end of nodS of USDA257 render the strain incapable of nodulating Leucaena diversifolia, L. leucocephala, and L. trichodes (Krishnan et al. 1992). On the other hand, NGR234, which possesses a functional nod $S U$ operon, readily nodulates these species (Table 1). Yet, at least three L. leucocephala isolates exist (94A3, 94A4, and WBM16) that apparently lack nodSU (Krishnan et al. 1992). This suggests either that N-methylated Nod factors (see Figure 2) are not always necessary for nodulation of Leucaena spp., or that another $N$-methyltransferase exists that has minimal homology to NodS. Nevertheless, adjunction of the $N$-methyl group to NodNGR factors is performed by the $N$-methyltransferase encoded by NodS (Jabbouri et al. 1995; Geelen et al. 1995).

$n o d Z$ and its role in fucosylation of Nod factors present an even greater paradox. All the usual G. max symbionts (B. elkanii, B. japonicum, $R$. fredii, but also NGR234) are fucosylated (see Hanin et al. 1998). Teleologically, one would have expected this fucose group (normally 2-O-methylfucose) to be a requirement for nodulation of $G$. max. Mutation of nodZ in the respective strains abolishes fucosylation of both $\mathrm{NodBj}$ and NodNGR factors but not nodulation of G. $\max$, however (Stacey et al. 1994; Quesada-Vincens et al. 1997). Even more puzzling is the observation that nonfucosylated NodBj factors isolated from a NodZ $Z^{-}$mutant are unable to deform root hairs of $G$. soja, a plant that nodZ mutants nevertheless nodulate (Stacey et al. 1994). In fact, the only $\mathrm{Nod}^{-}$phenotype observed so far concerns NGR $\Delta$ nodZ mutants that are $\mathrm{Nod}^{-}$on Pachyrhizus tuberosus (Quesada-Vincens et al. 1997), suggesting that fucosylated Nod factors are necessary for nodulation of this plant. As mentioned above, NodRf factors are also fucosylated (Fig. 2), and, on this basis, USDA257 would be expected to nodulate $P$. tuberosus, but this is not the case (Table 1). On $M$. atropurpureum, a NodZ ${ }^{-}$mutant of $B$. japonicum displays a delayed nodulation phenotype (Stacey et al. 1994), yet the same nodZ gene extends the host range of $R$. leguminosarum to include $M$. atropurpureum (Lopez-Lara et al. 1996). Mutation of nodZ has no effect on nodulation of $M$. atropurpureum by NGR234 (Quesada-Vincens et al. 1997).
Obviously, Nod-factor substituents alone are insufficient to explain host range. Host specificity can also be broken when Nod factors are present above certain "threshold" levels (Relić et al. 1994a). Probably, a complex interplay between the induction of nod genes by flavonoids, the excretion of variously substituted Nod factors, the relative and absolute Nod-factor levels, and feedback control of flavonoid accumulation (Schmidt et al. 1994) are all involved in nodulation of the different legumes.

\section{DISCUSSION}

\section{Broad host range.}

To the best of our knowledge, the host-range spectrum of individual Rhizobium strains has been the subject of only two other extensive investigations. The first, and certainly the most detailed, was that of Wilson (1939), who measured the abilities of 32 soil isolates to nodulate 160 different legumes. Broad-host-range rhizobia were found in both the Mimosaceae (present day Mimosoideae) and Fabaceae (Papilinoideae). All but two of the 32 isolates could nodulate more than 25 species, and three were extremely promiscuous in their symbiotic capacities. These included a Rhizobium isolate from Albizia julibrissin that nodulated 92 species, a Rhizobium isolate from Desmodium canadense that nodulated 93 species, and a presumed Bradyrhizobium isolate from Mucuna pruriens DC. subsp. deeringiana (Bort.) P. Hanelt (= Stizolobium deeringianum Bort.) that nodulated 105 legume species. Unfortunately, these strains were not examined further and, as far as we know, they have been lost. Bradyrhizobium sp. strain CB756, which was isolated from Macrotyloma africanum ( $\mathrm{R}$. Wilczek) Verdc. growing in Zimbabwe, is another broad-hostrange organism. Although widely available, it has been tested on a relatively restricted set of legumes, for which only positively responding species were recorded (Diatloff and Date 1978).

A simplified comparison of the nodulation data available for these four rhizobia plus USDA257 and NGR234 is presented in Table 4. All six strains appear to be adapted for nodulation of members of the Mimosoid tribes Acacieae, Ingae, and Mimoseae as well as the Papilionoid tribes Desmodieae, Genisteae, Robinieae, but especially the Phaseoleae. Given the affinity of both NGR234 and USDA257 for the Desmodieael Phaseoleae, it might have been expected that broad-hostrange rhizobia would have preferred hosts within these tribes. Two out of the six strains failed to nodulate V. unguiculata, which is widely recognized as being promiscuous (Table 3; Lewin et al. 1987b; Hernandez-Lucas et al. 1995). None of the strains nodulated all plants, nor was any one plant nodulated by all strains. Nevertheless, NGR234 nodulated more of these "broad-host-range hosts" than any other rhizobia so far studied, and in this sense is the most promiscuous known rhizobia. Perhaps not surprisingly, in view of the hypothesis that defects in the host account for the lack of nodulation, none of the promiscuous rhizobia were able to overcome the recalcitrance of caesalpinioid legumes such as Bauhinia, Caesalpinia, and Cassia spp. to nodulate (see Allen and Allen 1981).

\section{Molecular basis of broad host range.}

Perhaps the most striking observation presented here is not that both bacteria have exceptionally broad host ranges, but 
that every plant that is nodulated by $R$. fredii USDA257 is also host to Rhizobium sp. strain NGR234. The antithesis is not true: USDA257 only nodulates approximately 58\% of the NGR234 hosts. And this is the conundrum: if USDA257 nodulates an exact subset of the NGR234 hosts and excretes an exact subset of NodNGR factors (see Figure 2), why cannot Nod-factor requirements be assigned to specific legumes? Obviously, correlations exist but they are far from perfect, and Nod-factor levels also play a role. Wild-type USDA257 secretes only one fortieth the amount of Nod factors produced by NGR234 (Relić et al. 1994a). Perhaps a combination of different levels of various Nod factors is responsible for the nodulation patterns.

Another intriguing observation is that if only one of the two bacteria forms an effective (i.e., nitrogen-fixing) association, it is almost always NGR234. The only exceptions to this rule concern Apios americana, G. max, and G. soja, where there is a reversal of the nitrogen fixation phenotypes: USDA257 is Fix $^{+}$while NGR234 is Fix ${ }^{-}$. Again, the reasons for these differences are not clear. Since nod genes are only expressed early in the symbiosis (Fellay et al. 1995b), it is unlikely that Nod factors themselves are responsible for the $\mathrm{Fix}^{+} / \mathrm{Fix}^{-}$phenotypes. Rhizobia are known to possess genes that permit full development of bacteroids and hence nodules (Wilson et al. 1987; Müller et al. 1995a, 1995b). Perhaps some of these differ between USDA257 and NGR234. Another possibility concerns the presence of genes homologous to the type three protein secretion system (TTSS) in both bacteria (Balatti et al. 1995; Freiberg et al. 1997; Gu et al. 1997; Viprey et al. 1998; Viprey et al., in press). The TTSS is an essential component of pathogenicity in both animal (e.g., Salmonella, Shigella, Yersinia) and plant (Erwinia, Pseudomonas, Ralstonia, and Xanthomonas) pathogens. Indications that genes encoding part of the TTSS are present in USDA257 developed from the work of Meinhardt et al. (1993). This and later studies showed that the nolXWTUV locus is responsible for the flavonoidinduced excretion (Krishnan and Pueppke 1993; Krishnan et al. 1995) of at least five proteins (Krishnan and Pueppke 1993). Mutation of nolXWTUV has cultivar- and host-specific effects on nodulation of certain legumes (Krishnan and Pueppke 1994; Kovács et al. 1995; Bellato et al. 1997). Sequence analysis of pNGR234a revealed that all genes necessary for elaboration of the TTSS are present in NGR234 (Freiberg et al. 1997). Insertional inactivation of several of them abolishes not only secretion of two proteins, but severely alters their ability to nodulate Pachyrhizus tuberosus (PhaseoleaeGlycininae) and Tephrosia vogelii (Millettieae) (Viprey et al. 199). It is thus possible that protein secretion is an important component of effectiveness in nitrogen fixation, and that differences in either the TTSS machinery itself or the exported proteins could help explain the variation in $\mathrm{Fix}^{+} / \mathrm{Fix}^{-}$phenotypes of USDA257 and NGR234.

\section{Evolutionary implications.}

It is even more difficult to explain the close genetic relationship between USDA257 and NGR234. Rhizobium populations are often heterogenous (e.g., see Broughton et al. 1987), yet no essential genes were found when genomic DNA of USDA257 was subtracted from that of NGR234 (Perret et al. 1994). DNA sequence homologies even extend to the nodulation genes. Only $19 \mathrm{bp}$ of the $3.08-\mathrm{kb} \operatorname{nodABC}$ loci are different between the two strains (Relić et al. 1994b). Obviously, similar nod genes direct the synthesis of comparable Nod factors, and these are part of the molecular basis of the nested host ranges. Still, this does not explain the evolutionary pressures that lead to broad host range. Experiments with different Vigna spp. and cultivars showed that both strains are more "cowpea-like" than bacteria (normally slow-growing Bradyrhizobium spp.) of the "cowpea" miscellany. Hosts of the "cowpea miscellany" that include a range of tropical and subtropical Phaseoleae and Desmodieae are also the preferred hosts of both bacteria. In other words, two different bacteria (USDA257 and NGR234) isolated from different genera ( $G$. soja and Lablab purpureus), at widely separated locations (China and Papua New Guinea), and at least a decade apart, acquired the ability to nodulate a group of Old World and Central American plants (Doyle et al. 1997).

Whether the ability of plants to nodulate arose once or a number of times during evolution is a matter of some controversy. Sprent and Sprent (1990) and Doyle (1994) suggest that it may have arisen several times within the Leguminosae. In contrast, Solitis et al. (1995) wrote of "the likelihood of only a single origin of the predisposition for root-nodule symbioses in angiosperms ...."-a radically opposed idea. Of course, our limited data are insufficient to resolve this conflict, yet Figure 3 suggests that both NGR234 and USDA257 have acquired the ability to nodulate three distinct groups of plants, in the Caesalpiniodieae, Mimosoideae, and Papilionoideae. In the Papilionoideae this ability arose early, and was maintained through to the Amorpheae, Robinieae, Indigofereae, Phaseoleae, and Desmodieae. Once acquired, the capacity to symbiose with promiscuous rhizobia was apparently lost in only five tribes. Of these, it is difficult to draw conclusions on the Abreae and Adesmieae, since only one and two plants, respectively, were tested. It seems clear, however, that tribes P19 to P21 (Vicieae, Cicereae, and Trifolieae) have lost the ability to nodulate with NGR234 or USDA257. Since the microsymbionts of these plants generally secrete fewer, lessmodified Nod factors, albeit with polyunsaturated acyl chains, this implies that narrow host range is a specialization that developed for certain plants in restricted niches.

It is possible to argue that Figure 3, which is based on morphological rather than molecular data, does not present the true relationships between the tribes. Accordingly, we sought molecular evidence of tribal associations, particularly those involving the crucial loss of nodulation that may have occurred between the Galegeae and the tribes Cicereae, Trifolieae, and Vicieae. Unfortunately, although much sequence data is now being published, it tends to deal with specific genera rather than whole subfamilies. Also, as Doyle et al. (1997) point out: "The chloroplast genome has provided . . . weak support for many clades in the [ribulose-1,5-bisphosphate carboxylase/oxygenase] $r b c \mathrm{~L}$ tree suggests that data from additional genes will be required to construct rigourous hypotheses from some groups [of the Leguminosae]" (words in brackets have been added for clarity). In other words, it is not possible, at the present moment, to replace Figure 3 with one constructed from purely molecular data. Nevertheless, Doyle et al. (1997) present a molecular analysis of the tribal relationships among the Papilionoideae. These are shown in Figure 4, along with the capacity of the tribes to nodulate with NGR234 and USDA257. Although the Cicereae were not included in 
Doyle et al.'s analysis, their data confirm our finding (Fig. 3) that the ability to nodulate with NGR234 or USDA257 has been lost in temperate, herbaceous legumes (their observation that the Galageae did not give rise to the Trifolieae and Vicieae is irrelevant to this argument).

It thus seems likely that symbiotic promiscuity is ancestral to restricted host range. Support for this hypothesis comes from the observation that NGR234/USDA257 also nodulate Parasponia andersonii (Ulmaceae). Parasponia spp. are small trees (up to $15 \mathrm{~m}$ high) that grow as pioneer plants in mountain areas of Indonesia, Malaysia, and Papua New Guinea (Akkermans and van Dijk 1981). In other words, ParasponiaRhizobium symbioses evolved in NGR234's habitat. As there is a direct correlation between solar energy input and species diversity (Roy et al. 1998), tropical regions are probably driving evolution. In this scenario, bacteria like NGR234/ USDA257 would have been the first to intimately associate with legumes.

Exactly what symbiotic genes, and therefore Nod factors, were necessary for these ancestral interactions is not apparent from the present data. Clearly, those additional Nod-factor substituents that NGR234 produces but USDA257 lacks are like "baroque" decorations-they enhance the general appeal of NGR234, but they are not necessary for basic promiscuity. Enzymes encoded by nod $S$ (the $N$-methyltransferase), nod $U$ (the 6-O-carbamoyl transferase, nolO (the 3- [or 4-]-Ocarbamoyl transferase), nolL (an acetyltransferase), noeE (a sulfotransferase), and noeI (a 2-O-methyltransferase) simply extend an already broad host range. Insertional mutation of nodZ, which encodes the fucosyltransferase, suggests that it is also a "baroque" gene, since the mutants are only $\mathrm{Nod}^{-}$on Pachyrhizus tuberosus (Quesada-Vincens et al. 1997).

If this logic reflects evolution, then the ancestral Nod factors were simply oligomers of chitin (probably trimers to pentamers), $\mathrm{N}$-acylated with a $\mathrm{C} 18$ fatty acid, and were able to nodulate many plants. Modifications followed that in the case of NGR234 allowed it to nodulate even more plants. Northern migration of USDA257 was accompanied, however, by point mutations and genome re-arrangements that restricted its host range. Such changes, when they occurred in the nodSU operon, for example, deprived USDA257 of the ability to secrete $\mathrm{N}$-methylated and 6-O-carbomoylated Nod factors, and thus the capacity to nodulate Leucaena spp. (Krishnan et al. 1992).

\section{MATERIALS AND METHODS}

The seeds listed in Table 1 were sterilized by immersion in concentrated $\mathrm{H}_{2} \mathrm{SO}_{4}$ for times ranging from $5 \mathrm{~min}$ (e.g., Glycine, Phaseolus, and Vigna spp.) to $4 \mathrm{~h}$ (Delonix, Erythrina, Robinia, etc.). They were then washed three times with sterile water, rinsed in $5 \%$ ( vol/vol) $\mathrm{H}_{2} \mathrm{O}_{2}$ for 5 to $10 \mathrm{~min}$, washed in sterile water, and placed on $\mathrm{B} \& \mathrm{D}$ agar (Broughton and Dilworth 1971) to germinate at $26^{\circ} \mathrm{C}$. Special treatments were necessary to ensure germination of certain species. As examples, the embryo had to be freed by dissection from seeds possessing very thick testas (e.g., Delonix - performed after sterilization and imbibition), cold treatments were necessary with others (e.g., Vicia spp.), while heat treatment (immersion in boiling water) was used with Swainsona. Immersion in sterile water for a few days after sterilization, followed by transfer to agar plates, helped germination of some small-seeded legumes (e.g., Hippocrepis ciliata). When the radicals were approximately $2 \mathrm{~cm}$ long, the seedlings were planted into washed vermiculite held in Leonard jars of three different sizes. Magenta jars (250 ml capacity) were used for small seedlings (Lewin et al. 1990), modified wine bottle assemblies (volume $=750 \mathrm{ml}$ ) were used for medium to large seeds (Arachis, Vicia faba, etc.) (Broughton and John 1979), while Leonard jars made from 2.5-liter laboratory reagent bottles were used for extremely large seeds (e.g., Castanospermum, Cynometra, Millettia megasperma, etc.). All plants were raised at a day temperature of $30^{\circ} \mathrm{C}$, a night temperature of $20^{\circ} \mathrm{C}$, and a light phase of $16 \mathrm{~h}$ (including a 1-h stepped "sunrise" and a 1-h stepped "sunset"). The intensity of illumination was $350 \mu \mathrm{E}$ $\mathrm{s}^{-1} \mathrm{~m}^{-2}$ PAR). Germinating seeds were inoculated with approximately $1 \times 10^{7} \log$-phase cells of one of the bacteria listed in Table 5 at planting (Pueppke 1983) or upon establishment (Lewin et al. 1990). Given the large number of legumes involved, and the paucity of information available on their rhizobial requirements, it was not possible to include positively nodulating controls for all plants in the experimental design. Rather, with the exception of the Medicago and Vigna spp., we simply asked whether either of the test bacteria could nodulate the plants listed in Table 1 (noninoculated plants served as negative controls). Since the rhizobial requirements of Medicago and Vigna spp. are well known, and as many species were tested, positive controls were inoculated with $R$. meliloti RCR2011 and B. japonicum USDA110, respectively. Each experiment was replicated, with one to 10 plants per treatment, depending on the availability of seeds and the size of the seedlings. Most nodulation experiments were performed at least twice. Plants were grown until the "controls" displayed clear symptoms of nitrogen deficiency (yellowing of the leaves, stunted growth), which varied between approximately 1 and 6 months, depending on the size of the seeds and the initial growth rate (Desmodium and Castonospermum represent the extremes). At harvest, the roots were examined for the presence/absence of nodules. Plants were listed as $\mathrm{Nod}^{-}$when there was no apparent response to inoculation. $\mathrm{Nod}^{+}$was used to describe outgrowths/nodules that, irrespective of the internal structure, lacked leghemoglobin. Fix ${ }^{+}$nodules possessed organized internal structures and bacteroids. Mature $\mathrm{Fix}^{+}$nodules were red in color, fixed nitrogen, and caused the leaves to green.

\section{ACKNOWLEDGMENTS}

We are deeply indebted to G. P. Lewis, J. I. Sprent, and R. M. Polhill for their extensive help in preparing the manuscript. Also, we wish to thank N. Boukli, M. D. Crisp, E. Cocking, R. A. Date, J. Dénarié, J. J. Doyle, P. L. Gepts, D. Gerber, the late A. Giovannini, A. Ikram, R. Jesinger, D. Kuykendall, T. Nasim, V. Našinec and family, C. E. Pankhurst, W. Rawlings, B. Relić, S. Relić, G. Stacey, S. Stone, A. Stork, M. J. Trinick, G. Webster, C.-H. Wong, and J. Zairi, for their invaluable assistance with many aspects of this work. Help in obtaining seeds was graciously provided by A. Arambarri (ULA), W. J. Blackmon (HBU), J. Bone (KRG), A. J. Bourne (AHA), F. J. de Bruijn (MLU), V. Buozov and A. Delkov (FSB), L. Davis (BMU), J. Fairlamb (NMA), M. L. Farrar (FBA), W. Fehr and J. Orf (AAU), T. Frisk (FSC), A. M. Ghanekar (IPI), C. Gardiner (ACA), D. Gerber and F. N. F. Nicollin (UGC), A. M. Ghanekar and M. H. Mengesha (IPI), P. H. Graham and M. J. Sadowsky (SSU), J. P. Gowela (FZM), P. M. Gresshoff (PKU), W. Haines (AOA), J. Hanson (IAE), M. Hybl (OCC), T. Hymowitz (PUU), A. Ikram (GKM), D. Kishinevsky (VBI), G. Lachard and R. Tripod (CGC), E. Ledesma (BTC), B. L. Maass, A. Ortiz, and R. Schultze-Kraft (CCC), S. Mitchell and H. Osborn (WSA), T. Muller 
(NHZ), R. Nelson (UUU), B. Pengelly (ASA), K. Poulsen (DHD), S. Ratnam (IFS), W. Rawlings (ROG), P. Recher (FDA), U. Sankawa (PTJ), S. P. Soteriou (FNC), S. Stone (UGC), D. Stout (WPU), M. Stronati (PPA), I. Suhirman (UBR), K. Tybirk (BAD), G. A. White (PBU), D. M. Wilson (WDA), C.-H. Wong (PPM), J. C. Wynne (CRU), N. D. Young (BSU), J. Zairi (PPM), and Z.-M. Zhang (HBC). Financial support was provided by the U.S. Department of Agriculture and the U.S. National Science Foundation (to S. G. P.), the Fonds national suisse de la recherche scientifique, the Erna och Victor Hasselblads Stiftelse (to W. J. B.), and the Université de Genève. Costs of publication were defrayed by grants from the Fonds Topali (administered by the Section de Biologie, Université de Genève) and the Fonds Marthe Seidl-Hentsch (administered by the Société Académique de Genève).

\section{LITERATURE CITED}

Akkermans, A. D. L., Abdulkadir, S., and Trinick, M. J. 1978. N N$_{2}$ fixing root nodules in Ulmaceae: Parasponia or (and) Trema spp. Plant Soil 49:711-715.

Akkermans, A. D. L., and van Dijk, C. 1981. Non-leguminous rootnodule symbiosis with actinomycetes and Rhizobium. Pages 57-103 in: Nitrogen Fixation. I. Ecology. W. J. Broughton, ed. Oxford University Press, Oxford.

Allen, O. N., and Allen, E. K. 1936. Root nodule bacteria of some tropical leguminous plants: I. Cross-inoculation studies with Vigna sinensis L. Soil Sci. 42:61-77.

Allen, O. N., and Allen, E. K. 1940. Response of the peanut plant to inoculation with rhizobia with special reference to morphological development of the nodules. Bot. Gaz. 102:121-142.

Allen, O. N., and Allen, E. K. 1981. The Leguminosae. A Source Book of Characteristics, Uses, and Nodulation. University of Wisconsin Press, Madison.

Arsenijević-Maksimović, I., Broughton, W. J., and Krause, A. 1997. Rhizobia modulate root-hair-specific expression of extensin genes. Mol. Plant-Microbe Interact. 10:95-101.

Arumuganathan, K., and Earle, E. D. 1991. Estimation of nuclear DNA content of plants by flow cytometry. Plant Mol. Biol. Rep. 9:208-219.

Athar, M. 1993. Nodulation status of some legume trees of Pakistan. Nitrogen Fixing Tree Res. Reports 11:32-34.

Athar, M. 1996a. Observations on nodulation status of some papilinoid species of potential agricultural and forestry value from Sacramento Valley, California. Taiwania 41:301-308.

Athar, M. 1996b. Nodulation status of some legume species from Cache Valley and Northern Utah. Phytologia 8:145-150.

Athar, M. 1997. A qualitative study of the nodulating ability of legumes of Pakistan. List 5. Acta Soc. Bot. Pol. 66:1-5.

Bachem, C. W. B., Banfalvi, Z., Kondorosi, E., Schell, J., and Kondorosi, A. 1986. Identification of host-range determinants in the Rhizobium sp. MPIK3030. Mol. Gen. Genet. 203:42-48.

Bachem, C. W. B., Kondorosi, E., Banfalvi, Z., Horvath, B., Kondorosi, A., and Schell, J. 1985. Identification and cloning of nodulation genes from the wide host range Rhizobium strain MPIK3030. Mol. Gen. Genet. 199:271-278.

Balatti, P. A., Kovács, L. G., Krishnan, H. B., and Pueppke, S. G. 1995. Rhizobium sp. NGR234 contains a functional copy of the soybean cultivar specificity locus, nolXWBTUV. Mol. Plant-Microbe Interact. 8:693-699.

Balatti, P. A., and Pueppke, S. G. 1990. Nodulation of soybean by a transposon-mutant of Rhizobium fredii USDA257 is subject to competitive nodulation blocking by other rhizobia. Plant Physiol. 94: 1276-1281.

Barker, D. G., Bianchi, S., Blondon, F., Dattée, Y., Duc, G., Essad, S., Flament, P., Gallusci, P., Génier, G., Guy, P., Muel, X., Tourneur, J., Dénarié, J., and Huguet, T. 1990. Medicago truncatula, a model plant for studying the molecular genetics of the Rhizobium-legume symbiosis. Plant Mol. Biol. Rep. 8:40-49.

Barneby, R. C. 1998. Silk Tree, Guanacaste, Monkey's Earing. A Generic System for the Synandrous Mimosaceae of the Americas. Part III. Calliandra. Mem. N. Y. Bot. Gard. 74.

Bassam, B. J., Rolfe, B. G., and Djordjevic, M. A. 1986. Macroptilium atropurpureum (siratro) host specificity genes are linked to a nodDlike gene in the broad host range Rhizobium strain NGR234. Mol. Gen. Genet. 203:49-57.

Bec-Ferté, M. P., Krishnan, H. B., Promé, D., Savagnac, A., Pueppke, S.
G., and Promé, J.-C. 1994. Structures of nodulation factors from the nitrogen-fixing symbiont Rhizobium fredii USDA257. Biochemistry 33:11782-11788

Bellato, C., Krishnan, H. B., Cubo, T., Temprano, F., and Pueppke, S. G. 1997. The soybean cultivar specificity gene nolX is present, expressed in a $n o d D$-dependent manner, and of symbiotic significance in cultivar-nonspecific strains of Rhizobium (Sinorhizobium) fredii. Microbiology (Cambridge) 143:1381-1388.

Berck, S., Perret, X., Quesada-Vincens, D., Promé, J.-C., Broughton, W. J., and Jabbouri, S. 1999. NolL of Rhizobium sp. strain NGR234 is required for $O$-acetyltransferase activity. J. Bacteriol. 181:957-964.

Beyerinck, M. W. 1888a. Die Bacterien der Papilionaceen-Knöllchen. Bot. Zeitung 46:725-740.

Beyerinck, M. W. 1888b. Die Bacterien der Papilionaceen-Knöllchen. Bot. Zeitung 46:741-750.

Beyerinck, M. W. 1888c. Die Bacterien der Papilionaceen-Knöllchen. Bot. Zeitung 46:757-771.

Beyerinck, M. W. 1888d. Die Bacterien der Papilionaceen-Knöllchen. Bot. Zeitung 46:781-790

Beyerinck, M. W. 1888e. Die Bacterien der Papilionaceen-Knöllchen. Bot. Zeitung 46:797-804

Beyerinck, M. W. 1890. Künstliche Infection von Vicia faba mit Bacillus radicicola. Ernährungsbedingungen dieser Bacterie. Bot. Zeitung 52:837-843.

Breteler, F. J. 1995. The boundary between Amherstieae and Detariae (Caesalpinioideae). Pages 53-61 in: Advances in Legume Systematics. 7. Phylogeny. M. Crisp and J. J. Doyle, eds. Royal Botanic Gardens, Kew, England.

Broughton, W. J., Bohlool, B. B., Shaw, C. H., Bohnert, H. J., and Pankhurst, C. E. 1985. Conserved plasmid/chromosome sequences in fastand slow-growing rhizobia that nodulate the same plant. Arch. Microbiol. 141:14-21.

Broughton, W. J., and Dilworth, M. J. 1971. Control of leghaemoglobin synthesis in snake beans. Biochem. J. 125:1075-1080.

Broughton, W. J., Dilworth, M. J., and Passmore, I. K. 1972. Base ratio determination using unpurified DNA. Anal. Biochem. 46:164-172.

Broughton, W. J., Heycke, N., Meyer z. A., H., and Pankhurst, C. E. 1984. Plasmid-linked nif and "nod" genes in fast-growing rhizobia that nodulate Glycine max, Psophocarpus tetragonolobus, and Vigna unguiculata. Proc. Natl. Acad. Sci. USA 81:3093-3097.

Broughton, W. J., Heycke, N., Priefer, U., Schneider, G.-M., and Stanley, J. 1987. Ecological genetics of Rhizobium meliloti: Diversity and competitive dominance. FEMS Microbiol. Lett. 40:245-249.

Broughton, W. J., Hoh, C. H., Behm, C. A., and Tung, H. F. 1978. Development of the nitrogen fixing apparatus in the legumes Centrosema pubescens Benth. and Vigna unguiculata L. Walp. Planta 139:183-192.

Broughton, W. J. and John, C. K. 1979. Rhizobia in tropical legumes. III. Experimentation and supply in Malaysia 1927-1976. Pages 113136 in: Soil Microbiology and Plant Nutrition. W. J. Broughton, C. K. John, J. C. Rajaro, and B. Lim, eds. University of Malaya Press, Kuala Lumpur, Malaysia.

Broughton, W. J., Wong, C.-H., Lewin, A., Myint, H., Meyer z. A., H., Dowling, D. N., and Simon, R. 1986. Identification of Rhizobium plasmid sequences involved in recognition of Psophocarpus, Vigna, and other legumes. J. Cell Biol. 102:1173-1182.

Carroll, W. R. 1934. A study of Rhizobium species in relation to nodule formation on the roots of Florida legumes. I. Soil Sci. 37:117-135.

Chandler, M. R. 1978. Some observations on the infection of Arachis hypogaea L. by Rhizobium. J. Exp. Bot. 29:749-755.

Chandler, M. R., Date, R. A., and Roughley, R. J. 1982. Infection and root-nodule development in Stylosanthes species by Rhizobium. J. Exp. Bot. 33:47-57.

Chen, W. X., Li, G. S., Qi, Y. L., Wang, E. T., Yuan, H. L., and Li, J. L. 1991. Rhizobium huakuii sp. nov. isolated from the root nodules of Astragalus sinicus. Int. J. Syst. Bacteriol. 41:275-280.

Chua, K.-J., Pankhurst, C. E., MacDonald, P. E., Hopcroft, D. H., Jarvis, B. D. W., and Scott, D. B. 1985. Isolation and characterization of Tn-5 induced symbiotic mutants of Rhizobium loti. J. Bacteriol. 162:335-343.

Cohn, J., Day, R. B., and Stacey, G. 1998. Legume nodule organogenesis. Trends Plant Sci. 3:105-110.

Corby, H. D. L. 1988. Types of nodules and their distribution among the Leguminosae. Kirkia 13:53-123.

Corby, H. D. L., Polhill, R. M., and Sprent, J. I. 1983. Taxonomy. Pages 1-35 in: Nitrogen Fixation. Vol. 3. Legumes. W. J. Broughton, ed. 
Clarendon Press, Oxford.

Cordeiro, L., and Sprent, J. I. 1996. Some aspects of nodule anatomy of Dalbergia nigra inoculated with rhizobia from members of the tribes Dalbergieae and Millettieae (Tephrosieae). Naturalia (Sao Paulo) 21:23-30.

Crisp, M. D., and Weston, P. H. 1987. Cladistics and legume systematics. Pages 65-130 in: Advances in Legume Systematics. Part 3. C. H. Stirton, ed. Royal Botanic Garden, Kew, England.

Dart, P. J. 1977. Infection and development of leguminous nodules. Pages 367-472 in: A Treatise on Dinitrogen Fixation. Vol. 3. R. W. F. Hardy and W. S. Silver, eds. Wiley Press, New York.

de Faria, S. M., Lewis, G. P., Sprent, J. I., and Sutherland, J. M. 1989. Occurrence of nodulation in the Leguminosae. New Phytol. 121:607-619.

de Souza Moreira, F. M., da Silva, M. F., and de Faria, S. M. 1992. Occurrence of nodulation in legume species in the Amazon region of Brazil. New Phytol. 121:563-570.

Dénarié. J., Debellé, F., and Promé, J.-C. 1996. Rhizobium lipo-chitooligosaccharide nodulation factors: Signalling molecules mediating recognition and morphogenesis. Annu. Rev. Biochem. 65:503-535.

Dénarié. J., Debellé, F., and Rosenberg, C. 1992. Signalling and host range variation in nodulation. Annu. Rev. Microbiol. 46:497-531.

Diatloff, A., and Date, R. A. 1978. CB756 and the broad spectrum strain concept. Rhizobium Newsl. 23:17-19.

Dilworth, M. J. 1969. The plant as genetic determinant of leghaemoglobin production in the legume root nodule. Biochim. Biophys. Acta 184:432-441

Dilworth, M. J., Arwas, R., McKay, I. A., Saroso, S., and Glenn, A. R. 1986. Pentose metabolism in Rhizobium leguminosarum MNF300 and in cowpea Rhizobium NGR234. J. Gen. Microbiol. 132:2733-2742.

Doyle, J. J. 1994. Phylogeny of the legume family: An approach to understanding the origins of nodulation. Annu. Rev. Ecol. Syst. 25:325-49.

Doyle, J. J., Doyle, J. L., Ballenger, J. A., Dickson, E. E., Kajita, T., and Ohashi, H. 1997. A phylogeny of the chloroplast gene RBCL in the Leguminosae: Taxonomic correlations and insights into the evolution of nodulation. Am. J. Bot. 84:541-554.

Dreyfus, B. L., and Dommergues, Y. R. 1981. Nodulation of Acacia species by fast- and slow-growing tropical strains of Rhizobium. Appl. Environ. Microbiol. 41:97-99.

Fellay, R., Perret, X., Viprey, V., Broughton, W. J., and Brenner, S. 1995a. Organisation of host-inducible transcripts on the symbiotic plasmid of Rhizobium sp. NGR234. Mol. Microbiol. 16:657-667.

Fellay, R., Rochepeau, P., Relić, B., and Broughton, W. J. 1995b. Signals to and emanating from Rhizobium largely control symbiotic specificity. Pages 199-220 in: Pathogenesis and Host Specificity in Plant Diseases: Histopathological, Biochemical, Genetic and Molecular Bases. Vol. 1: Prokaryotes. U. S. Singh, R. P. Singh, and K. Kohmoto, eds. Pergamon/Elsevier Science, Oxford.

Frank, B. 1879. Ueber die Parasiten in den Wurzelanschwellungen der Papilionaceeen. Bot. Zeitung 24:377-388

Fred, E. B., Baldwin, I. L., and McCoy, E. 1932. Root Nodule Bacteria and Leguminous Plants. University of Wisconsin Stud. Sci. 5, Madison.

Freiberg, C., Fellay, R., Bairoch, A., Broughton, W. J., Rosenthal, A., and Perret, X. 1997. Molecular basis of symbiosis between Rhizobium and legumes. Nature 387:394-401.

Fuchsius, L. 1542. De Historia Stirpium Commentarii Insignes. Basel.

Geelen, D., Leyman, B., Mergaert, P., Klarskov, K., van Montagu, M., Geremia, R., and Holsters, M. 1995. NodS is an S-adenosyl-Lmethionine-dependent methyltransferase that methylates chitooligosaccharides deacylated at the non-reducing end. Mol. Microbiol. 17: 387-397.

Gehring, C. A., Irving, H. R., Kabbara, A. A., Parish, R. W., Boukli, N. M., and Broughton, W. J. 1997. Rapid, plateau-like increases in intracellular free calcium are associated with Nod-factor induced root-hair deformation. Mol. Plant-Microbe Interact. 10:791-802.

Göttfert, M. 1993. Regulation and function of rhizobial nodulation genes. FEMS Microbiol. Rev. 104:39-64.

Gu, F., Balatti, P. A., Krishnan, H. B., and Pueppke, S. G. 1997. Characterization of the overlapping promoters of $n o l B$ and $n o l W$, two soybean cultivar specificity genes from Rhizobium fredii strain USDA257. Mol. Plant-Microbe Interact. 10:138-141.

Handberg, K., and Stougard, J. 1992. Lotus japonicus, an autogamous, diploid legume species for classical and molecular genetics. Plant J. 2: 487-496.

Hanin, M., Jabbouri, S., Broughton, W. J., Fellay, R., and Quesada-
Vincens, D. 1999. Molecular aspects of host-specific nodulation. Pages 1-37 in: Plant-Microbe Interactions. Vol. 4. G. Stacey and N. T. Keen, eds. American Phytopathological Society, St. Paul, MN.

Hanin, M., Jabbouri, S., Quesada-Vincens, D., Freiberg, C., Perret, X., Promé, J.-C., Broughton, W. J., and Fellay, R. 1997. Sulphation of Rhizobium sp. NGR234 Nod factors is dependent on noeE, a new host-specificity gene. Mol. Microbiol. 24:1119-1129.

Harrier, L. A., Whitty, P. W., Sutherland, J. M., and Sprent, J. I. 1997. Phenetic investigation of species of Acacia leguminosae using morphological and molecular markers. Plant Syst. Evol. 205:27-51.

Hellriegel, H. 1886. Welche Stickstoffquellen stehen der Pflanze zu Gebote? Landwirtsch. Vers.-Stn. Dresden 33:464-465.

Hellriegel, H., and Wilfarth, H. 1888. Untersuchungen über die Stickstoffnahrung der Gramineen und Leguminosen. Beilageheft zu der Zeitschrift des Vereins für die Rübenzucker-Industrie des Deutschen Reiches, Buchdruckerei der "Post," Kayssler \& Co., Berlin.

Hernandez-Lucas, I., Segovia, L., Martinez-Romero, E., and Pueppke, S. G. 1995. Phylogenetic relationships and host range of Rhizobium spp. that nodulate Phaseolus vulgaris. Appl. Environ. Microbiol. 61: 2775-2779.

Heron, D. S., Érsek, T., Krishnan, H. B., and Pueppke, S. G. 1989. Nodulation mutants of Rhizobium fredii USDA257. Mol. PlantMicrobe Interact. 2:4-10.

Heron, D. S., and Pueppke, S. G. 1984. Mode of infection, nodulation specificity, and indigenous plasmids of 11 fast-growing Rhizobium japonicum strains. J. Bacteriol. 160:1061-1066.

Hiltner, L. 1900. Über die Ursachen, welche die Grösse, Zahl, Stellung und Wirkung der Wurzelknöllchen der Leguminosen bedingen. Arb. Biol. Abt. Land- Forstwirthsch., Kais. Gesundheits., Berlin 1:177-222.

Hiltner, L., and Störmer, K. 1903. Neue Untersuchungen über die Wurzelknöllchen der Leguminosen und deren Erreger. Arb. Biol. Abt. Land- Forstwirthsch., Kais. Gesundheits., Berlin 3:151-307.

Horvath, B., Bachem, C. W. B., Schell, J., and Kondorosi, A. 1987. Host-specific regulation of nodulation genes in Rhizobium is mediated by a plant-signal, interacting with the nodD gene product. EMBO J. 6 : 841-848.

Hymowitz, T., and Newell, C. A. 1980. Taxonomy, speciation, domestication, dissemination, germplasm resources and variation in the genus Glycine. Pages 251-264 in: Advances in Legume Science. R. J. Summerfield and A. H. Bunting, eds. Royal Botanic Gardens, Kew, England.

Jabbouri, S., Fellay, R., Talmont, F., Kamalaprija, P., Burger, U., Relić, B., Promé, J.-C., and Broughton, W. J. 1995. Involvement of nodS in $\mathrm{N}$-methylation and nodU in 6-O-carbamoylation of Rhizobium sp. NGR234 Nod-factors. J. Biol. Chem. 270:22968-22973.

Jabbouri, S., Relić, B., Hanin, M., Kamalaprija, P., Burger, U., Promé, D., Promé, J.-C., and Broughton, W. J. 1998. nolO and noeI (HsnIII) of Rhizobium sp. NGR234 are involved in 3-O-carbamoylation and 2$O$-methylation of Nod-factors. J. Biol. Chem. 279:12047-12055.

James, E. K., Shaw, J. E., Catellan, A. J., de Faria, S. M., and Sprent, J. I. 1993. The infection of aquatic and terrestrial Neptunia species by Rhizobium. Page 351 in: New Horizons in Nitrogen Fixation. R. Palacios, J. Mora, and W. E. Newton, eds. Kluwer Academic Pub., Dordrecht, The Netherlands

Jiang, Q., and Gresshoff, P. M. 1997. Classical and molecular genetics of the model legume Lotus japonicus. Mol. Plant-Microbe Interact. 10: 59-68.

Keyser, H. H., and Griffin, R. F. 1987. Beltsville Rhizobium Culture Catalogue. U.S. Agric. Res. Serv., Beltsville, MA.

Keyser, H. H., Hu, T. S., Bohlool, B. B., and Weber, D. F. 1982. Fastgrowing rhizobia isolated from root nodules of soybean. Science 215: 1631-1632.

Kondorosi, A., Pankhurst, C. E., Broughton, W. J., and Banfalvi, Z. 1982. Mobilization of a Rhizobium meliloti megaplasmid carrying nodulation and nitrogen fixation genes into other rhizobia and Agrobacterium. Mol. Gen. Genet. 188:433-439.

Kovács, L. G., Balatti, P. A., Krishnan, H. B., and Pueppke, S. G. 1995. Transcriptional organization and expression of nolXWBTUV, a locus that regulates cultivar-specific nodulation of soybean by Rhizobium fredii USDA257. Mol. Microbiol. 17:923-933.

Krause, A., Sigrist, C. J. A., Dehning, I., Sommer, H., and Broughton, W. J. 1994. Accumulation of transcripts encoding a lipid transfer-like protein during deformation of nodulation-competent Vigna unguiculata root hairs. Mol. Plant-Microbe Interact. 7:411-418.

Krishnan, H. B., Kuo, C.-L., and Pueppke, S. G. 1995. Elaboration of 
flavonoid-induced proteins by the nitrogen-fixing soybean symbiont Rhizobium fredii is regulated by both nodD1 and nodD2, and is dependent on the cultivar-specificity locus, nolXWBTUV. Microbiology (Cambridge) 141:2245-2251.

Krishnan, H. B., Lewin, A., Fellay, R., Broughton, W. J., and Pueppke, S. G. 1992. Differential expression of nodS accounts for the varied abilities of Rhizobium fredii USDA257 and Rhizobium sp. NGR234 to nodulate Leucaena spp. Mol. Microbiol. 6:3321-3330.

Krishnan, H. B., and Pueppke, S. G. 1993. Flavonoid inducers of nodulation genes stimulate Rhizobium fredii USDA257 to export proteins into the environment. Mol. Plant-Microbe Interact. 6:107-113.

Krishnan, H. B., and Pueppke, S. G. 1994. Cultivar-specificity genes of the nitrogen-fixing soybean symbiont, Rhizobium fredii USDA257, also regulate nodulation of Erythrina spp. Am. J. Bot. 81:38-45.

Kuykendall, L. D., and Elkan, G. H. 1976. Rhizobium japonicum derivatives differing in nitrogen-fixing efficiency and carbohydrate utilization. Appl. Environ. Microbiol. 32:511-519.

Kuykendall, L. D., Saxena, B., Devine, T. E., and Udell, S. E. 1992. Genetic diversity in Bradyrhizobium japonicum Jordan 1982 and a proposal for Bradyrhizobium elkanii sp. nov. Can. J. Microbiol. 38:501-505.

Leonard, L. T. 1943. A simple assembly for use in the testing of cultures of rhizobia. J. Bacteriol. 45:523-525.

Lerouge, P., Roche, P., Faucher, C., Maillet, F., Truchet, G., Promé, J. C., and Dénarié, J. 1990. Symbiotic host-specificity of Rhizobium meliloti is determined by a suphated and acylated glucosamine oligosaccharide signal. Nature 344:781-784.

Lewin, A., Cervantes, E., Wong, C.-H., and Broughton, W. J. 1990. nodSU, two new nod-genes of the broad host range Rhizobium strain NGR234 encode host-specific nodulation of the tropical tree Leucaena leucocephala. Mol. Plant-Microbe Interact. 5:317-326.

Lewin, A., Rosenberg, C., Meyer z. A., H., Wong, C.-H., Nelson, L., Manen, J.-F., Stanley, J., Dowling, D. N., Dénarié, J., and Broughton, W. J. 1987a. Multiple host-specificity loci of the broad host range Rhizobium sp. NGR 234 selected using the widely compatible legume Vigna unguiculata. Plant Mol. Biol. 8:447-459.

Lewin, A., Rosenberg, C., Stanley, J., Dowling, D. N., Manen, J.-F., Debellé, F., and Broughton, W. J. 1987b. Multiple host-specificity loci in the broad host-range Rhizobium NGR234. Pages 232-237 in: Molecular Genetics of Plant-Microbe Interactions. D. P. S. Verma and N. Brisson, eds. Martinus Nijhoff, Dordrecht, The Netherlands.

Lim, G., and Burton, J. C. 1982 Nodulation status of the Leguminosae. Pages 1-34 in: Nitrogen Fixation. 2 Rhizobium. W. J. Broughton, ed. Oxford University Press, Oxford.

Lim, G., and Ng, H. L. 1977. Root nodules of some tropical legumes in Singapore. Plant Soil 46:317-327.

Long, S. 1996. Rhizobium symbiosis: Nod factors in perspective. Plant Cell 8:1885-1898.

Lopez-Lara, I. M., Blok-Tip, L., Quinto, C., Garcia, M. L., Stacey, G., Bloemberg, G. V., Lamers, G. E. M., Lugtenberg, B. J. J., ThomasOates, J. E., and Spaink, H. P. 1996. NodZ of Bradyrhizobium extends the nodulation host range of Rhizobium by adding a fucosyl residue to nodulation factors. Mol. Microbiol. 21:397-408.

Lorquin, J., Lortet, G., Ferro, M., Méar, N., Dreyfus, B., Promé, J.-C., and Boivin, C. 1997a. Nod factors from Sinorhizobium saheli and S. teranga bv. sesbaniae are both arabinosylated and fucosylated, a structural feature specific to Sesbania rostrata symbionts. Mol. PlantMicrobe Interact. 10:879-890.

Lorquin, J., Lortet, G., Ferro, M., Méar, N., Promé, J.-C., and Boivin, C. 1997b. Sinorhizobium teranga bv. acaciae ORS1073 and Rhizobium sp. strain ORS1001, two distantly related Acacia-nodulating strains, produce similar Nod factors that are $\mathrm{O}$ carbamoylated, $\mathrm{N}$ methylated and mainly sulfated. J. Bacteriol. 179:3079-3083.

Lortet, G., Méar, N., Lorquin, J., Dreyfus, B., de Lajudie, P., Rosenberg, C., and Boivin, C. 1996. Nod factor thin-layer chromatography profiling as a tool to characterize symbiotic specificity of rhizobial strains: Application to Sinorhizobium saheli, S. teranga, and Rhizobium sp. strains isolated from Acacia and Sesbania. Mol. PlantMicrobe Interact. 9:736-747.

Mabberley, D. J. 1987. The Plant Book. Cambridge University Press, Cambridge.

Meijer, E. G. M., and Broughton, W. J. 1982. Biology of legumeRhizobium interactions in nodule formation. Pages 107-129 in: Molecular Biology of Plant Tumors. G. Kahl and J. S. Schell, eds. Academic Press, New York.
Meinhardt, L. W., Krishnan, H. B., Balatti, P. A., and Pueppke, S. G. 1993. Molecular cloning and characterization of a sym plasmid locus that regulates cultivar-specific nodulation of soybean by Rhizobium fredii USDA257. Mol. Microbiol. 9:17-29.

Mergaert, P., van Montagu, M., Promé, J.-C., and Holsters, M. 1993. Three unusual modifications, a D-arabinosyl, a $N$-methyl, and a carbomyl group, are present on the Nod factors of Azorhizobium caulinodans strain ORS571. Proc. Natl. Acad. Sci. USA 90:1551-1555.

Morrison, N. A., Cen, Y. H., Chen, H. C., Plazinski, J., Ridge, R., and Rolfe, B. G. 1984. Mobilization of a sym plasmid from a fast-growing cowpea Rhizobium strain. J. Bacteriol. 160:483-487.

Morrison, N. A., Hau, C. Y., Trinick, M. J., Shine, J., and Rolfe, B. G. 1983. Heat curing of a Sym plasmid in a fast-growing Rhizobium sp. that is able to nodulate legumes and the nonlegume Parasponia sp. J. Bacteriol. 153:527-531.

Morrison, N. A., Trinick, M. J., and Rolfe, B. G. 1986. Comparison of the host range of fast-growing $R$. japonicum strains with a fastgrowing isolate from Lablab. Plant Soil 92:313-317.

Müller, P., Ahrens, K., Keller, T., and Klaucke, A. 1995a. A TnphoA insertion within the Bradyrhizobium japonicum sipS gene, homologous to prokaryotic signal peptidases, results in extensive changes in the expression of PBM-specific nodulins of infected soybean (Glycine max) cells. Mol. Microbiol. 18:831-840.

Müller, P., Klaucke, A., and Wegel, E. 1995b. TnphoA-induced symbiotic mutants of Bradyrhizobium japonicum that impair cell and tissue differentiation of Glycine max nodules. Planta 197:163-175.

Nayudu, M., and Rolfe, B. G. 1987. Analysis of R-primes demonstrates that genes for broad host range nodulation of Rhizobium strain NGR234 are dispersed on the Sym plasmid. Mol. Gen. Genet. 206: 326-337.

Nobbe, F., Hiltner, L., and Schmid, E. 1895. Versuche über die Biologie der Knöllchenbakterien der Leguminosen, insbesondere über die Frage der Arteinheit derselben. Landwirtsch. Vers.-Stn. Dresden 45: 1-27.

Nobbe, F., Schmid, E., Hiltner, L., and Hotter, E. 1891. Versuche über die Stickstoff-Assimilation der Leguminosen. Landwirtsch. Vers.-Stn. Dresden 39:327-359.

Norris, D. O. 1956. Legumes and the Rhizobium symbiosis. Emp. J. Exp. Agric. 24:247-70.

Ogawa, J., Brierley, H. L., and Long, S. R. 1991. Analysis of Rhizobium meliloti nodulation mutant WL131: Novel insertion sequence ISRm3 in nodG and altered nodH protein product. J. Bacteriol. 173:3060-3065.

Padmanabhan, S., Hirtz, R.-D., and Broughton, W. J. 1989. Rhizobia in tropical legumes: Cultural characteristics of Bradyrhizobium and Rhizobium sp. Soil Biol. Biochem. 22:23-28.

Pankhurst, C. E., Broughton, W. J., Bachem, C., Kondorosi, E., and Kondorosi, A. 1983a. Identification of nitrogen fixation and nodulation genes on a large plasmid from a broad host range Rhizobium spp. Pages 169-176 in: Molecular Genetics of the Bacteria-Plant Interaction. A. Pühler, ed. Springer-Verlag, Berlin.

Pankhurst, C. E., Broughton, W. J., and Wieneke, U. 1983b. Transfer of an indigenous plasmid of Rhizobium loti to other rhizobia and Agrobacterium tumefaciens. J. Gen. Microbiol. 129:2535-2543.

Perret, X., Fellay, R., Bjourson, A. J., Cooper, J. E., Brenner, S., and Broughton, W. J. 1994. Subtraction hybridisation and shot-gun sequencing: A novel approach to identify symbiotic loci. Nucleic Acids Res . 22:1335-1341.

Perret, X., Freiberg, C., Rosenthal, A., Broughton, W. J., and Fellay, R. High-resolution transcriptional analysis of the symbiotic plasmid of Rhizobium sp. NGR234. Mol. Microbiol. (In press.)

Perret, X., Jabbouri, S., and Broughton, W. J. Molecular basis of symbiotic promiscuity. J. Bacteriol. (In press.)

Perret, X., Viprey, V., Freiberg, C., and Broughton, W. J. 1997. Structure and evolution of NGRRS-1, a complex, repeated element in the genome of Rhizobium sp. NGR234. J. Bacteriol. 179:7488-7496.

Polhill, R. M. 1994. Classification of the Leguminosae. Pages xvi-xxxvii in: Phytochemical Dictionary of the Leguminosae, Vol 1. Plants and Their Constituents. F. A. Bisby, J. Buckingham, and J. B. Harborne, eds. Chapman \& Hall, London.

Polhill, R. M., and Raven, P. H., eds. 1981. Advances in Legume Systematics. Parts 1 and 2. Royal Botanic Gardens, Kew, England.

Prazmowski, A. 1889. Das Wesen und die biologische Bedeutung der Wurzelknöllchen der Erbse. Bot. Centralblatt 39:356-362 (from Ber. Sitz. K. K. Akad. Wiss. Krakau 1889).

Prazmowski, A. 1890. Die Wurzelknöllchen der Erbse. Landwirtsch. 
Vers.-Stn., Berlin 37:161-238.

Price, N. P. J., Relić, B., Talmont, F., Lewin, A., Promé, D., Pueppke, S. G., Maillet, F., Dénarié, J., Promé, J.-C., and Broughton, W. J. 1992. Broad-host-range Rhizobium species NGR234 secretes a family of carbamoylated, and fucosylated, nodulation signals that are $O$ acetylated or sulphated. Mol. Microbiol. 6:3575-3584.

Pueppke, S. G. 1983. Rhizobium infection threads in root hairs of Glycine $\max$ (L.) Merr., Glycine soja Sieb. \& Zucc., and Vigna unguiculata (L.) Walp. Can. J. Microbiol. 29:69-76.

Quesada-Vincens, D., Fellay, R., Nasim, T., Viprey, V., Burger, U., Promé, J.-C., Broughton, W. J., and Jabbouri, S. 1997. Rhizobium sp. NGR234 NodZ protein is a fucosyltransferase. J. Bacteriol. 179: 5087-5093.

Relić, B., Perret, X., Estrada-Garcia, M. T., Kopcinska, J., Golinowski, W., Krishnan, H. B., Pueppke, S. G., and Broughton, W. J. 1994a. Nod-factors of Rhizobium are a key to the legume door. Mol. Microbiol. 13:171-178.

Relić, B., Staehelin, C., Fellay, R., Jabbouri, S., Boller, T., and Broughton, W. J. 1994b. Do Nod-factor levels play a role in host-specificity? Pages 69-75 in: Proc. Eur. Nitrog. Fix. Conf., 1st. G. B. Kiss and G. Endre, eds. Officina Press, Szeged, Hungary.

Relić, B., Talmont, F., Kopcinska, J., Golinowski, W., Promé, J.-C., and Broughton, W. J. 1993. Biological activity of Rhizobium sp. NGR234 Nod-factors on Macroptilium atropurpureum. Mol. Plant-Microbe Interact. 6:764-774.

Roche, P., Debellé, F., Maillet, F., Lerouge, P., Faucher, C., Truchet, G., Dénarié. J., and Promé, J.-C. 1991a. Molecular basis of symbiotic specificity in Rhizobium meliloti: nodH and nodPQ genes encode the sulfation of lipo-oligosaccharie signals. Cell 67:1131-1143.

Roche, P., Lerouge, P., Ponthus, C., and Promé, J.-C. 1991b. Structural determination of bacterial nodulation factors involved in the Rhizobium meliloti-alfalfa symbiosis. J. Biol. Chem. 266:10933-10940.

Rosenberg, C., Boistard, P., Dénarié, J., and Casse-Delbart, F. 1981. Genes controlling early and late functions in symbiosis are located on a megaplasmid in Rhizobium meliloti. Mol. Gen. Genet. 184:326-333.

Roy, K., Jablonski, D., Valentine, J. W., and Rosenberg, G. 1998. Marine latitudinal diversity gradients: Tests of causal hypotheses. Proc. Natl. Acad. Sci. USA 95:3699-3702.

Schmidt, P. E., Broughton, W. J., and Werner, D. 1994. Nod factors of Bradyrhizobium japonicum and Rhizobium sp. NGR234 induce flavonoid accumulation in soybean root exudate. Mol. Plant-Microbe Interact. 7:384-390.

Schultze, M., Kondorosi, E., Ratet, P., Buiré, M., and Kondorosi, A. 1994. Cell and molecular biology of Rhizobium-plant interactions. Int. Rev. Cytol. 156:1-75.

Scott, D. B., Young, C. A., Collins-Emerson, J. M., Terzaghi, E. A., Rockman, E. S., Lewis, P. E., and Pankhurst, C. E. 1996. Novel and complex chromosomal arrangement of Rhizobium loti nodulation genes. Mol. Plant-Microbe Interact. 9:187-197.

Small, E., and Jomphe, M. 1989. A synopsis of the genus Medicago (Leguminosae). Can. J. Bot. 67:3260-3294.

Soltis, D. E., Soltis, P. S., Morgan, D. R., Swensen, S. M., Mullin, B. C., Dowd, J. M., and Martin, P. G. 1995. Chloroplast gene sequence data suggest a single origin of the predisposition for symbiotic nitrogen fixation in angiosperms. Proc. Natl. Acad. Sci. USA 92:2647-2651.

Spaink, H. P. 1996. Regulation of plant morphogenesis by lipo-chitin oligosaccharides. Crit. Rev. Plant Sci. 15:559-582.

Spaink, H. P., Sheeley, D. M., van Brussel, A. A. N., Glushka, J., York, W. S., Tak, T., Kennedy, E. P., Reinhold, V. N., and Lugtenberg, B. J. J. 1991. A novel highly unsaturated fatty acid moiety of lipo-oligosaccharide signals determines host specificity of Rhizobium. Nature $354: 125-120$

Sprent, J. I. 1980. Root nodule anatomy, type of export product and evolutionary origin of some Leguminosae. Plant Cell Environ. 3:35-43.

Sprent, J. I. 1994. Nitrogen acquisition systems in the Leguminosae. Pages 1-16 in: Advances in Legume Systematics 5. The Nitrogen Factor. J. I. Sprent and D. McKey, eds. Royal Botanic Gardens, Kew, England.

Sprent, J. I., and Sprent, P. 1990. Nitrogen Fixing Organisms. Chapman and Hall, New York.

Sprent, J. I., Sutherland, J. M., and de Faria, S. M. 1989. Structure and function of nodules from woody legumes. Pages 559-578 in: Advances in Legume Systematics. C. H. Stirton and J. L. Zarucchi, eds. Monogr. Syst. Bot., Missouri Bot. Gard. Vol. 29.

Stacey, G., Luka, S., Sanjuan, J., Banfalvi, Z., Nieuwkoop, A. J., Chun,
J. Y., Forsberg, L. S., and Carlson. R. 1994. nodZ, a unique hostspecific nodulation gene, is involved in the fucosylation of the lipooligosaccharide nodulation signal of Bradyrhizobium japonicum. J. Bacteriol. 176:620-633.

Stanley, J., Dowling, D. N., and Broughton, W. J. 1988. Cloning of hemA from Rhizobium sp. NGR234 and symbiotic phenotype of a gene-directed mutant in diverse legume genera. Mol. Gen. Genet. 215:32-37.

Stiller, J., Martirani, L., Tuppale, S., Chian, R.-J., Chiurazzi, M., and Gresshoff, P. 1997. High frequency transformation and regeneration of transgenic plants in the model legume Lotus japonicus. J. Exp. Bot. 48:1357-1365

Stowers, M. D., and Eaglesham, A. R. 1984. Physiological and symbiotic characteristics of fast-growing Rhizobium japonicum. Plant Soil 77:3-14.

Sutherland, J. M., McInroy, S. G., James, E. K., and Naisbitt, T. 1994. Nodule structure with special reference to the tribes Sophoreae, Genisteae, and Thermopsideae. Pages 41-55 in: Advances in Legume Systematics 5. The Nitrogen Factor. J. I. Sprent and D. McKey, eds. Royal Botanic Gardens, Kew, England.

Szczyglowski, K., Shaw, R. S., Wopereis, J., Copeland, S., Hamburger, D., Kasiborski, B., Dazzo, F. B., and de Bruijn, F. J. 1998. Nodule organogenesis and symbiotic mutants of the model legume Lotus japonicus. Mol. Plant-Microbe Interact. 11:684-697.

Taylor, J. M., and Crisp, M. D. 1992. A revision of Chorizema (Leguminosae: Mirbelieae). Aust. Syst. Bot. 5:249-335.

Trese, A., and Pueppke, S. G. 1991. Cloning of cowpea (Vigna unguiculata) genes that are regulated during initiation of nodulation. Mol. Plant-Microbe Interact. 4:46-51.

Trinick, M. J. 1973. Symbiosis between Rhizobium and the non-legume, Trema aspera. Nature 244:459-460.

Trinick, M. J. 1980. Relationships amongst the fast-growing rhizobia of Lablab purpureus, Leucaena leucocephala, Mimosa spp., Acacia farnesiana and Sesbania grandiflora and their affinities with other rhizobial groups. J. Appl. Bacteriol. 49:39-53.

Trinick, M. J. 1982. Biology. Pages 1-34 in: Nitrogen Fixation. 2. Rhizobium. W. J. Broughton, ed. Oxford University Press, Oxford.

Trinick, M. J., and Galbraith, J. 1980. The Rhizobium requirements of the non-legume Parasponia in relationship to the cross-inoculation concept of legumes. New Phytol. 86:17-26.

Truchet, G., Barker, D. G., Camut, S., de Billy, F., Vasse, J., and Huguet, T. 1989. Alfalfa nodulation in the absence of Rhizobium. Mol. Gen. Genet. 219:65-68.

Vassal, J. 1981. Tribe 4. Acacieae Benth. (1842), nom. conserv. prop. Pages 169-171 in: Advances in Legume Systematics. Part 1. R. M Polhill and P. H. Raven, eds. Royal Botanic Gardens, Kew, England.

Viprey, V., Del Greco, A., Golinowski, W., Broughton, W. J., and Perret, X. 1998. Symbiotic implications of type III protein secretion machinery in Rhizobium. Mol. Microbiol. 28:1381-1389.

Viprey, V., Perret, X., and Broughton, W. J. Host-plant invasion by rhizobia. In: Subcellular Biochemistry Vol. 10. Bacterial Invasion into Eukaryotic Cells. J. Hacker and T. Olschläger, eds. Plenum Pub., London. (In press.)

Whitty, P. W., Powell, W., and Sprent, J. I. 1994. Molecular separation of genera in Cassinae (Leguminosae), and analysis of variation in the nodulation species of Chamaecrista. Mol. Ecol. 3:507-515.

Williams, W. M., Hoh, C.-H., Lenz, F., and Broughton, W. J. 1988. Rhizobia in tropical legumes: Environmental factors and the reduction of nitrogen. Soil Biol. Biochem. 20:667-675.

Wilson, J. K. 1939. Leguminous plants and their associated organisms. Cornell Univ. Agric. Exp. Stn. Mem. 221. Cornell University Press, Ithaca, NY.

Wilson, K. J., Nambiar, P. T. C., Anjaiah, V., and Ausubel, F. M. 1987. Isolation and characterisation of Bradyrhizobium sp. (Arachis) strain NC92 mutants with host-specific defects in nodulation and nitrogen fixation. J. Bacteriol. 169:2177-2186.

Wong, C.-H., Pankhurst, C. E., Kondorosi, A., and Broughton, W. J. 1983. Morphology of root-nodules and nodule-like structures formed by Rhizobium and Agrobacterium strains containing a $R$. meliloti megaplasmid. J. Cell Biol. 97:787-794.

Wong, C.-H., Patchamuthu, R., Meyer z. A., H., Pankhurst, C. E., and Broughton, W. J. 1988. Rhizobia in tropical legumes: Ineffective nodulation of Arachis hypogaea L. by fast-growing strains. Soil Biol. Biochem. 20:677-681. 